\title{
1998 Tier Two \\ Emergency and \\ Hazardous Chemical \\ Inventory \\ Emergency Planning and \\ Community Right-To-Know Act \\ Section 312
}

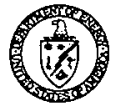

United States

Department of Energy

Richland, Washington

Approved for Public Release 
TRADEMAPK DISCLAMMER

Reference herein to any specific commercial product, process, or service by trade name, trademark, manufacturer, or otherwise, does not necessarily constitute of imply its endorsement, recommendation, or favoring by the United States Government or any agency thereof or its contractors or subcontractors.

This report has been reproduced from the best available copy. Available in paper copy and microfiche.

Available to the U.S. Department of Energy and its contractors from

Office of Sclentific and Technical Information

P.O. Box 62

Oak Ridge, TN 37831

(515) 576-8401

Available to the public from the U.S. Department of Commerce Netional Technical Information Service

5285 Port Royal Road

Springfield, VA 22161

(703) $487-4650$

Printed in the United Statere of Amarice

DISCLM-5.CHP (8-91) 
DOE/RL-99-16

UC-600

\section{Tier Two Emergency and Hazardous Chemical Inventory \\ Emergency Planning and Community Right-To-Know Act Section 312}




\section{PREFACE}

The Hanford Site covers approximately 1,450 square kilometers (560 square miles) of land that is owned by the U.S. Government and managed by the U.S. Department of Energy, Richland Operations Office (DOE-RL). The Hanford Site is located northwest of the city of Richland, Washington. The city of Richland adjoins the southeastern portion of the Hanford Site boundary and is the nearest population center.

Activities on the Hanford Site are centralized in mumerically designated areas. The 100 Areas, Iocated along the Columbia River, contain deactivated reactors. The processing units are in the 200 Areas, which are on a plateau approximately 11 kilometers (7 miles) from the Columbia River. The 300 Area, located adjacent to and north of Richland, contains research and development laboratories. The 400 Area, 8 kilometers (5 miles) northwest of the 300 Area, contains the Fast Flux Test Facility previously used for testing liquid metal reactor systems. Adjacent to the north of Richland, the 1100 Area contains offices associated with administration, maintenance, transportation, and materials procurement and distribution. The 600 Area covers all locations not specifically given an area designation.

This Tier Two Emergency and Hazardous Chemical Inventory report contains information pertaining to hazardous chemicals managed by DOE-RL and its contractors on the Hanford Site. It does not include chemicals maintained in support of activities conducted by others on lands covered by leases, use permits, easements, and other agreements whereby land is used by parties other than DOE-RL. For example, this report does not include chemicals stored on state owned or leased lands (including the burial ground operated by US Ecology, Inc.), lands owned or used by the Bonneville Power Administration (including the Midway Substation and the Ashe Substation), lands used by the National Science Foundation (the Laser Interferometer Gravitational-Wave Observatory), lands leased to the Washington Public Power Supply System, Johnson Controls, Inc. (boilers operated for steam production), and R. H. Smith Company (gas stations), or similarly leased lands not under the management of DOE-RL. 


\author{
DLTE: JAN 04 DE3 \\ REPLY TO \\ ATTM OF: EAP:EBD 93-RPA-07I \\ SUBJEET: OELEGATION OF SIGNATURE AUTHORITY FOR THE EMERGENCY AND \\ HAZARDOUS CHEMICAL INYENTORY REPORT (SARA 312)
}

10: James D. Bauer, Acting Progrim Manager

Office of Environmental Assurance, Permits, and Policy

I hereby delegata signature authority for the Emergency and Hazardous Chemical Inventory Report (SARA 312) to the Program Hanager, Ofirica of Environmental Assurance, Permits, and Policy.

Pursuant to 40 CFR Section 370.41, Tier II Emergency and Hazardous Chemical Inventory form, part (b), signature authority may be delegated to the manager's officially designated representative. This memorandum constitutes formal delegation of such authority to the Program Manager, Office of Environmental Assurance, Permits, and Policy.

$$
\text { Sincerely, }
$$

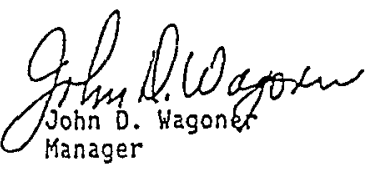


Washington Community Right-To-Know \#:

\section{TIER TWO}

EMERGENCY

AND

HAZARDOUS

CHEMICAL

INVENTORY

Specific
Information
by Chemical acility Identification

WA7890008967

Page 1

of 70

Name U. S. DERARTMENT OF ENERGY - HANFORD SITE

Street 825 JADWIN AVENUE

City RICHIAND County BENTON State WA Zip 99352

SIC code \begin{tabular}{|l|l|l|l|}
9 & 9 & 9 & 9 \\
\hline
\end{tabular} \begin{tabular}{|l|l|l|l|l|l|} 
Dun \& Brad & Number 0 & 3 \\
\hline
\end{tabular}$-$\begin{tabular}{|l|l|l|l|l|}
\hline & 4 & 5 & 8 & 6 \\
\hline
\end{tabular}

$\begin{array}{lll}\text { For } & & \\ \text { Official } & \text { ID\# } & \\ \text { Use } & & \\ \text { Only } & \text { Date } & \\ \text { Received } & & \\ \end{array}$

Name U. $S$. DERARTMENT OF FNERGY

Phone (509) 376-7411

Mail Address_R,O, BOX 550, RICHLAND WA 29352

\section{Emergency Contac:}

Name PATROI, ORERATIONS CENTER

Phone (509) 373-3800

Name

Phone ( )
Title SHIFT COMMANDER 24 Hr. Phone $(509) \quad 373-3800$

Title $24 \mathrm{Hr}$. Phone
Important: Read all instructions before completing form

Chemical Description

CAS \begin{tabular}{l|l|l|l|l|l|l|l|l|}
\hline & & 1 & 3 & 4 & 4 \\
\hline
\end{tabular} Chem. Name ALUMINUM OXIDE.

Check all $\underset{\text { Pure }}{\bigotimes} \underset{\text { Mix }}{\bigotimes} \underset{\text { Solid }}{\bigotimes} \underset{\text { Liquid }}{\bigotimes} \underset{\text { Gas }}{\square} \underset{\text { EHS }}{\square}$

EHS Name

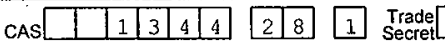

Chem. Name ALUMINUM OXIDE

$\begin{array}{llllll}\text { Check all } & \bigotimes & \bigotimes & \bigotimes & \bigotimes & \square\end{array}$

EHS Name

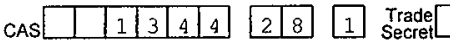

Chem. Name ALUMINUM_OXIDE,

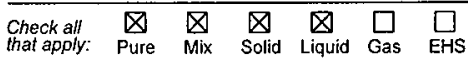

EHS Name
Reporting Period: Fro
Physucal
and Haalth
Hazards
(Chock all that apply)

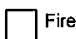

Sudden Release

of Pressure

Reactivity

Immediate (acute)

$\mathrm{X}$ Delayed (chronic)

\section{Fire}

Sudden Release

of Pressure

Reactivity

Immediate (acute)

$x$ Delayed (chronic)

\section{$\square$ Fire}

Sudden Release of Pressure

Reactivity

Immediate (acute)

$x$ Delayed (chronic)

Inventory January 1 to December 31,

\section{(1) Màx Daily}

$\begin{array}{ll}0 & 4 \\ \text { Amoút }\end{array}$

\begin{tabular}{|l|l|l|l|l|l|l}
\hline & 0 & 4 & Avg: Daily \\
Amount (codo)
\end{tabular}

\begin{tabular}{l|l|l}
\hline & 6 & \\
No of Days
\end{tabular}

Oni-Site (days)

\begin{tabular}{|l|l|}
\hline 0 & 4 \\
\hline
\end{tabular} Amox Daily (code)

\begin{tabular}{|l|l|l}
\hline 0 & 4 & Avg Daily \\
Amount (cod
\end{tabular}

\begin{tabular}{|l|l|l|l}
\hline 3 & 6 & 5 & No of Days \\
On-Site (days)
\end{tabular}

\begin{tabular}{|l|l|l}
\hline 0 & 4 & Max Daily \\
Amount (code)
\end{tabular}

014 Avg. Daily

Avg. Daily

\begin{tabular}{|l|l|l|l|l|l|l|l}
\hline 3 & 6 & 50. of Days \\
On-Site (days)
\end{tabular}

1998
$y-1$
$0 \quad s$

Che

Check if information below is identical to the information submitted last

Storage codes and Locations

(Non Confidential)

Storago Locations

\begin{tabular}{|l|l|l|l|l|l|l|l}
\hline F & 1 & 4 \\
\hline
\end{tabular}

K 114 1723N WAREHOUSE $100 N$ AREA

\begin{tabular}{l|l|l|l} 
E & 1 & 4 & 1900R WAREHOUSE.
\end{tabular}

\begin{tabular}{lll|l}
\hline E & 1 & 4 & 2101 HV WAREHOUSE
\end{tabular}

$100 D / D R$ AREA

\begin{tabular}{lll} 
I & 1 & 4 \\
\hline
\end{tabular}

200E AREA

\begin{tabular}{lllll}
\hline & 1 & 4 & $2101 \mathrm{M}$ WAREHOUSE \\
\hline
\end{tabular}

$200 E$ AREA 200E AREA

\begin{tabular}{|l|l|l|l|l|l}
\hline $\mathrm{K}$ & 1 & 4 \\
\hline
\end{tabular}

$\begin{array}{llllll}M & 1 & 4 & 2101 \mathrm{M} \text { BUILDING, ROOM 210 2OOE AREA }\end{array}$

\begin{tabular}{llll}
\hline$J$ & 1 & 4 \\
$221 T$ CANYON ___m_ 200
\end{tabular}

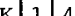

200W AREA

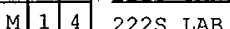
200W AREA

\begin{tabular}{l|l|l|l|l|l|l|l|l}
\hline$F$ & 1 & 4 & $222 \mathrm{SA}$ & $\mathrm{SAB}$
\end{tabular} 200W.AREA

\begin{tabular}{|l|l|l|l|l|l}
\hline$M$ & 1 & 4 \\
\hline$N$ & 1 & $422 S A$ & LAB & $200 \mathrm{~W}$ AREA
\end{tabular}

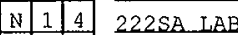
200W AREA

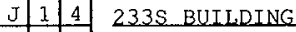
200W AREA

$\mathrm{E} \quad 1 \quad 4 \quad 234-57, \mathrm{BOOM} 116$ $200 W$ AREA

\begin{tabular}{lll|l}
$M$ & 1 & 4 & $234-52$. ROOM 202
\end{tabular} $200 W$ AREA 200W AREA and that based on $\mathrm{my}$

James E. Rasmussen, Director of Environmental

Assurance, Permits and Policy Division

Name and official title of owner/operator OR owner/operator's authorized representative

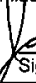

Signature $\angle 2$
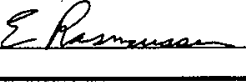

ren. moxnes
$02 / 26 / 99$ Date signed
Optional Attachments

$\mathrm{X}$ I have attached a site plan I have attached a list of site coordinate abbreviations

I have attached a description of dikes and other safeguard measures 


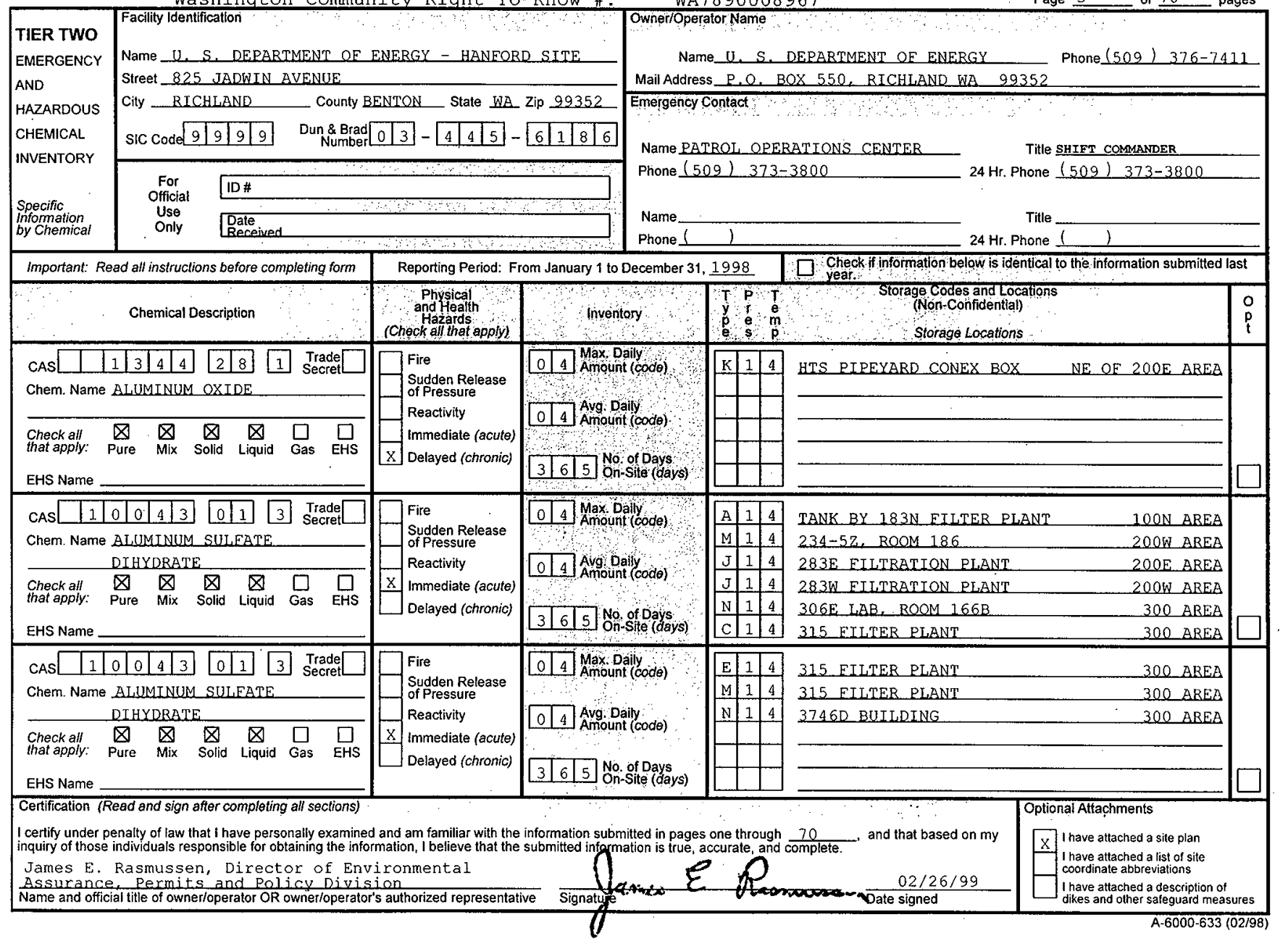


Washington Community Right-To-know \# :

\section{TIER TWO}

EMERGENCY

AND

HAZARDOUS

CHEMICAL

INVENTORY

Specific

information

by Chemical

important: $R$ ead

Important: Read all instructions before completing form Chemical Description

CAS \begin{tabular}{l|l|l|l|l|l|l|}
\hline & & & & & \\
Chem. Name AMMUNITION
\end{tabular}
Trade
Secret

Chem. Name AMMUNITION

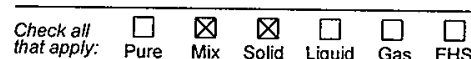

that apply:

EHS Name

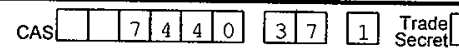

Chem. Name ARGON

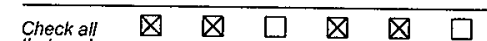

that apply: Pure Mix Solid Liquid Gas EHS

EHS Name

CAS \begin{tabular}{|l|l|l|l|l|l|l|l|}
\hline & & 7 & 4 & 4 & 0 \\
\hline 3 & 7 & 1 \\
\hline
\end{tabular}

Chem. Name $A R G O N$

\begin{tabular}{|c|c|c|c|c|c|}
\hline $\begin{array}{l}\text { Check all } \\
\text { that apply: }\end{array}$ & $\underset{\text { Pure }}{\triangle}$ & $\underset{M i x}{\otimes}$ & $\square_{\text {Solid }}$ & $\underset{\text { Liquid }}{\bigotimes}$ & $\mathrm{G}$ \\
\hline
\end{tabular}

EHS Name

(Certification (Read and sign after complefing all sections)

inquiry of those indivi of law that I have personally examined and am familiar with the information submitted in pages one through 70

inquiry of those individuals responsible for obtaining the information, I believe
James E. Rasmussen, Director of Environmental.
Assurance. Permits, and Policy Division.

IName and official title of owner/operator OR owner/operator's authorized representative Emergency Contact

Name

Phone ( )
Physical Hazards all that apply):

\begin{tabular}{|c|}
\hline Fire \\
\hline $\begin{array}{l}\text { Sudden Release } \\
\text { of Pressure }\end{array}$ \\
\hline Reactivity \\
\hline Immediate (acute) \\
\hline Delayed (chronic) \\
\hline
\end{tabular}

\begin{tabular}{|l|l}
$\mathrm{X}$ & $\begin{array}{l}\text { Fire } \\
\text { Sudden Release } \\
\text { of Pressure }\end{array}$
\end{tabular}

Reactivity

$\mathrm{X}$ Immediate (acute)

Delayed (chronic)

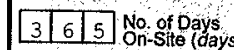

\begin{tabular}{|l|l|l|}
\hline 0 & 5 & Max Dally \\
\hline
\end{tabular}

Sudden Release

of Pressure

Reactivity

$\mathrm{x}$ Immediate (acute)

Delayed (chronic)

Avg. Dally \begin{tabular}{|l|l|l|l}
3 & 6 & 5 & No: of Days \\
On-Site (days)
\end{tabular}

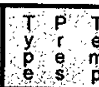

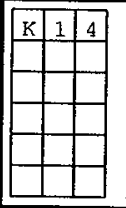

\begin{tabular}{|c|c|c|}
\hline $\mathrm{L}$ & 2 & 4 \\
\hline $\mathrm{L}$ & 2 & 4 \\
\hline $\mathrm{L}$ & 2 & 4 \\
\hline $\mathrm{I}$ & 2 & 4 \\
\hline $\mathrm{L}$ & 2 & 4 \\
\hline $\mathrm{L}$ & 2 & 4 \\
\hline
\end{tabular}

WA7890008967

Page 4 of 70 pages Owner/Operatior Name

Name_U. S. DERARTMENT OF ENERGY Phone (509) 376-7411 Mail Address _ P. O, BOX 550, RICHLAND WA. 99352

Name RATROL OPERATIONS CENTER

Phone (509) 373-3800

Title SHIFT COMMANDER

Title

$24 \mathrm{Hr}$. Phone

Reporting Period: From January 1 to December 31, 1998 G Check if information below is identlcal to the information submitted last

Storage Codes and Locations
(Non-Confidential)

Storage Locations

\begin{tabular}{|l|l|l|l|l}
\hline $\mathrm{L}$ & 2 & 4 \\
\hline
\end{tabular}

$100 K$ AREA

L 22 SHACK NE OF $105 \mathrm{KE}$

10OK AREA

\begin{tabular}{lll}
$\mathrm{L}$ & 2 & 4 \\
\hline & & 4 \\
\hline
\end{tabular}

$100 K$ AREA

\begin{tabular}{llll}
$\mathrm{L}$ & 2 & 4 \\
\hline
\end{tabular}

100K AREA

\begin{tabular}{lll}
$\mathrm{L}$ & 2 & 4 \\
\hline
\end{tabular}

1171. SHOP, DEACTIVATED

1100 AREA 1100 AREA

1512N GAS STORAGE $100 N$ AREA

$1515 \mathrm{~N}$ SHOP $100 N$ AREA

$165 \mathrm{KE}$ POWER CONTROL BUILDTNG 10OK AREA 100K AREA $1706 \mathrm{KE} \perp \mathrm{AB}$ 100K.AREA $1717 \mathrm{~K}$ WABEHOUSE $100 K$ KREB

$1724 \mathrm{~KB}$ GAS STORAGE BUILDING Optional Attachments

mitted infarmation is true, accurate, and complete and that based on my I have attached a list of site coordinate abbreviations 1 have attached a description of dikes and other safeguard measures A-6000-633 (02/98) 


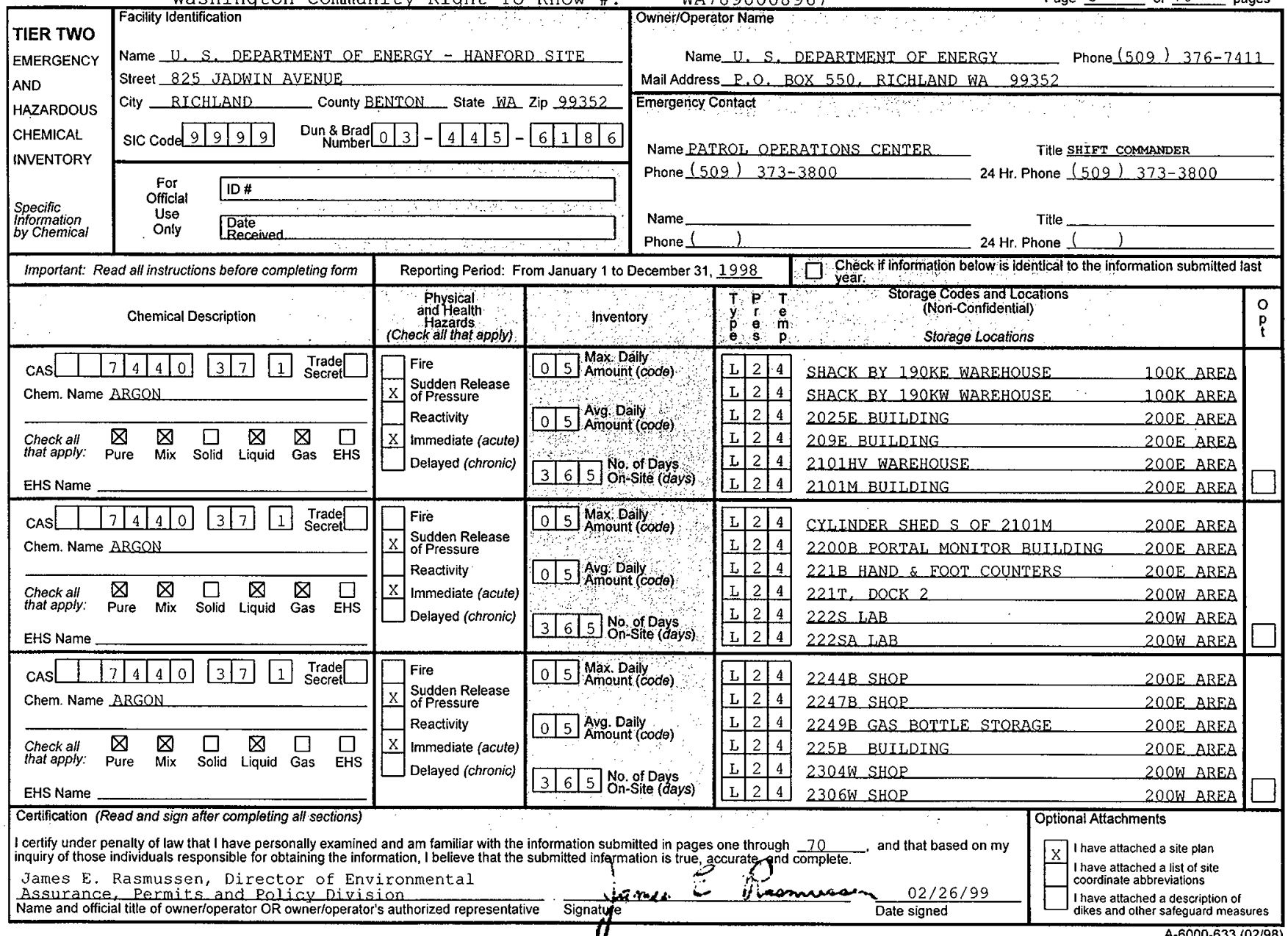


Washington Community Right-To-know \#:

\section{TIER TWO}

IEMERGENCY

IAND

IHAZARDOUS

(CHEMICAL

IINVENTORY

Specific

iinformation

lby Chemical Facility Ideritification

Name _U. S. DERARTMENT OF ENERGY - HANFORD SITE

Street 825 JADWIN AVENUE

City RICHLAND C County BENTON_L State WA Zip 99352

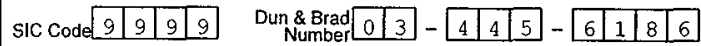

$\begin{array}{cl}\text { For } & \\ \text { Official } & \text { ID\# } \\ \text { Use } \\ \text { Only } & \text { Date } \\ \text { Raceived } & \end{array}$

WA7 890008967

Page 6 of 70 pages Owner/Operator Name

Name.U. S. DEPARTMENT OF ENERGY Mail Address P.O. BOX 550, RICHLAND WA 99352

Phone $(509) \quad 376-7411$ Emergency Contact

Name PATROL OPERATIONS CENTER Phone (509) 373-3800

Name Phone (
Title SHIFT COMMANDER $24 \mathrm{Hr}$. Phone $(509) \quad 373-3800$

Title $24 \mathrm{Hr}$. Phone $(1$
Important: Read all instructions before completing form

Chemical Description

Reporting Period: From January 1 to December 31, 1998

$$
\begin{gathered}
\text { Physical } \\
\text { and Health }
\end{gathered}
$$
and Health Hazards.
(Check all that apply)

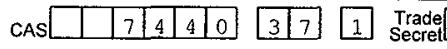
Chem. Name ARGON

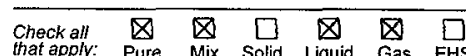
that apply: Pure Mix Solid Liquid Gas EHS EHS Name CAS \begin{tabular}{|l|l|l|l|l|l|l|l|}
\hline & & 7 & 4 & 4 & 0 \\
\hline
\end{tabular} Chem. Name ARGON

\begin{tabular}{|c|c|c|c|c|c|c|}
\hline $\begin{array}{l}\text { Check all } \\
\text { that apply: }\end{array}$ & $\underset{\text { Pure }}{\bigotimes}$ & $\underset{\text { Mix }}{\bigotimes}$ & $\underset{\text { Solid }}{\square}$ & $\underset{\text { Liquid }}{\bigotimes}$ & $\underset{\text { Gas }}{\bigotimes}$ & $\square$ \\
\hline
\end{tabular}

EHS Name

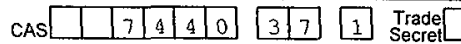
Chem. Name ARGON

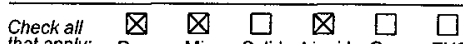
that apply: Pure Mix Solid Liquid Gas EHS

EHS Name (Certification (Read and sign after completing all sections)

\section{Tire}

$\mathrm{x}$ Sudden Release of Pressure

Reactivity

$\mathrm{X}$ Immediate (acute) Delayed (chronic)

\section{\begin{tabular}{|l|l}
\hline & $\begin{array}{l}\text { Fire } \\
\text { Sudden Release } \\
\text { of Pressure }\end{array}$ \\
\hline $\mathrm{X}$ &
\end{tabular} \\ of Pressure \\ Reactivity \\ X Immediate (acute) \\ Delayed (chronic)}

\begin{tabular}{|c|c|}
\hline & Fire \\
\hline $\mathrm{X}$ & $\begin{array}{l}\text { Sudden Release } \\
\text { of Pressure }\end{array}$ \\
\hline & Reactivity \\
\hline$x$ & Immediate (acute) \\
\hline
\end{tabular}

Delayed (chronic)

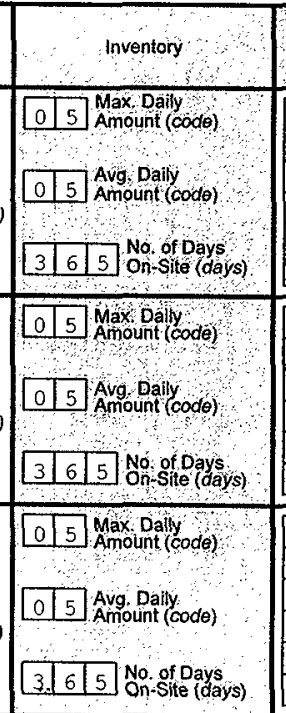

\begin{tabular}{|c|c|c|}
\hline & $\begin{array}{l}\mathrm{P} \\
\mathrm{e}\end{array}$ & $\ddot{\theta}$ \\
\hline $\mathrm{L}$ & 2 & 4 \\
\hline L & 2 & 4 \\
\hline $\mathbf{L}$ & 2 & 4 \\
\hline $\mathbf{L}$ & 2 & 4 \\
\hline $\mathrm{L}$ & 2 & 4 \\
\hline $\mathbf{L}$ & 2 & 4 \\
\hline $\mathrm{L}$ & 2 & 4 \\
\hline $\mathrm{L}_{4}$ & 21 & 4 \\
\hline $\mathbf{L}$ & 2 & 4 \\
\hline $\mathrm{L}$ & 2 & 4 \\
\hline $\mathrm{L}$ & 2 & 4 \\
\hline L & 2 & 4 \\
\hline $\mathrm{L}$ & 2 & 4 \\
\hline $\mathrm{L}$ & 2 & 4 \\
\hline L & 2 & 0 \\
\hline $\mathrm{L}$ & 2 & 4 \\
\hline $\mathrm{L}$ & 2 & 4 \\
\hline $\mathrm{L}$ & 2.1 & 4 \\
\hline
\end{tabular}
$\square$. Check Check if information below is identical to the information submitted last i.

(Non-Confidential)

Storage Locations

$2307 W$ SHOP $2309 \mathrm{~W}$ SHOP 2310W SHOP $2336 \mathrm{~W}$ PROCESS AREA $234-52$, ROOM 183 24IAN TANK FARM PORTAL MONITOR $200 \mathrm{E}$ ARF

241AP TANK FARM 200E AREA 241AW TANK FARM PORTBL MONITOR 200E_AREA 241AY TANK EARM PORTAL MONTTOR $200 E$ AREA 24IBX TANK FARM PORTAL MONITOR 20OE AREA 241C TANK FARM PORTAL MONITOR 200E AREA 241SX TANK EARM PQRTAL MONITOR 200W AREA $\square$ 241SY TANK FARM PORTAL MONITOR 200W AREA $241 T$ TANK EARM PORTAL MONITOR_.. 200W AREA 241TX TANK FARM PORTAL MONITOR 20OW AREA 2410 TANK FARM PORTAL MONITOR 200W AREA $242 A$ EVAPORATOR 242AC SHOP $200 E$ AREA 200E_AREA II certify under penalty of law that I have personally examined and am familiar with the information submitted in pages one through 70 James E. Rasmussen, Director of Environmental Assurance, Permits and Policy Division Name and official title of owner/operator OR owner/operator's authorized representative

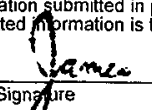


Washington Community Right-To-know \#

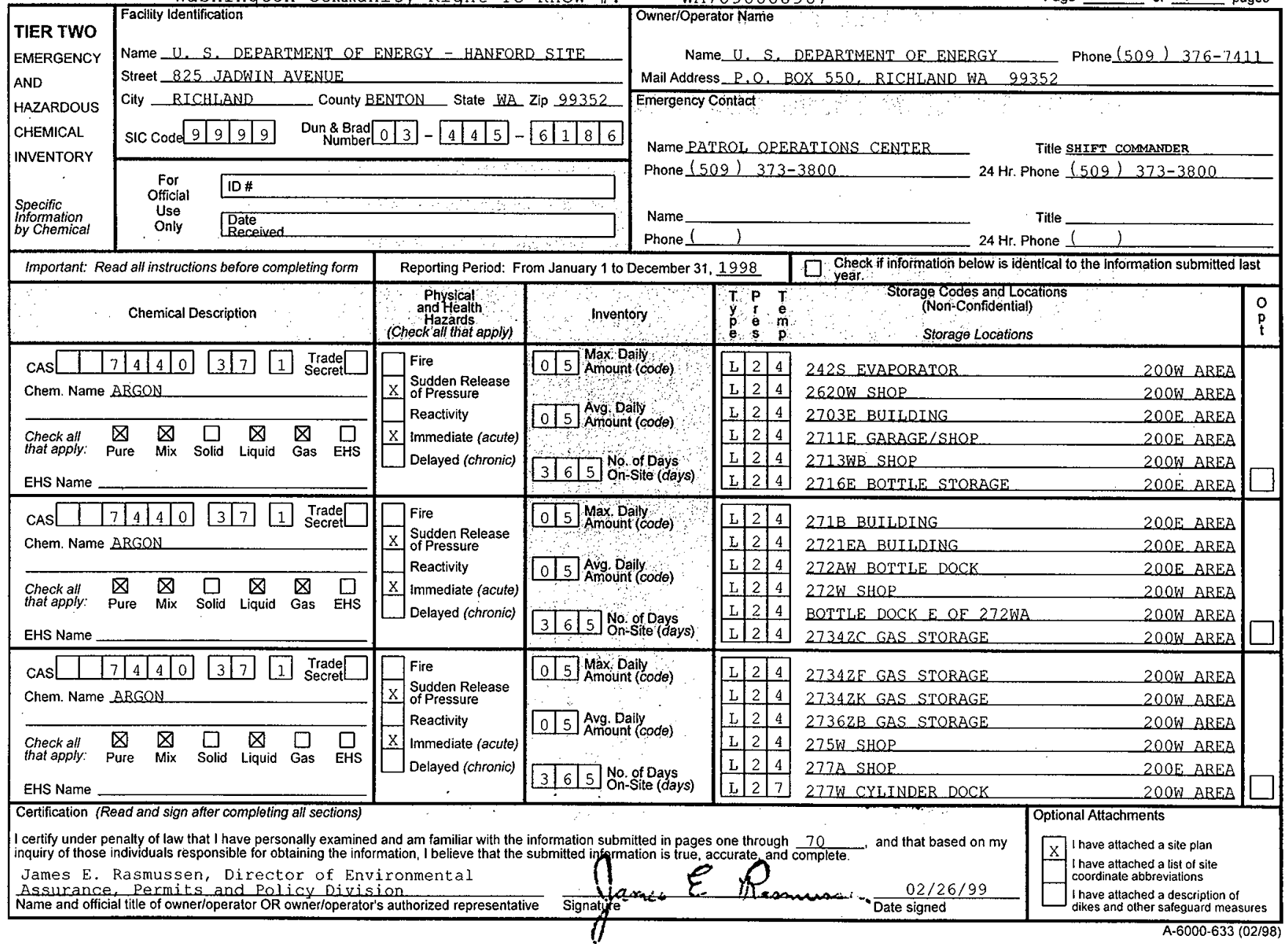




\section{TIER TWO} EMERGENCY AND HAZARDOUS CHEMICAL INVENTORY Specific
information
by Chemical Name U. S. DERARTMEN' OF ENERGY - HANFORD SITE Street 825 JADWIN AVENUE City BICHLAND County BENTON_ State WA Zip 99352

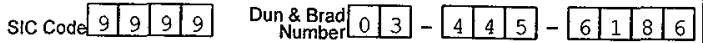

For
Official
Use
Only
Onate
Received

\section{Owner/Operator Namé}

Name_U. S. DEPARTMENT OF ENERGY Mail Address P. O. BOX 550, RICHLAND WA 99352

Phone (502) $376-7411$ Emergency.Contact

Name PATROL OPERATIONS CENTER Phone $(509) \quad 373-3800$ Name

Phone 5
Reporting Period: From January 1 to December 31, 1998 Phiysical
and Healt
Hazards
Check all that apply)
Inventon Fire

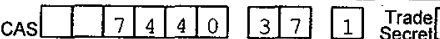
Chem. Name ARGON

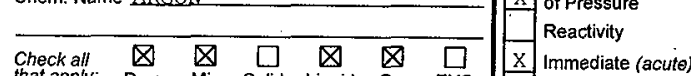
that apply: Pure Mix Solid Liquid Gas EHS $\square$ Delayed (chronic) EHS Name

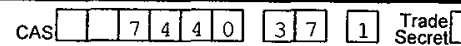
Chem. Name ARGON

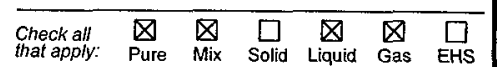
EHS Name CAS \begin{tabular}{l|l|l|l|l|l|l|l|l|}
\hline & & 7 & 4 & 4 & 0 \\
\hline 3 & 7 \\
\hline
\end{tabular} Chem. Name ARGON

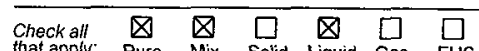
that apply: Pure Mix Solid Liquid Gas EHS EHS Name (Certification (Read and sign after completing all sections)

II certify under penalty of law that I have personally examined and am familiar with the information submitted in pages one through 70 inquiry of those individuals responsible for obtaining the information, I believe that the submitted information is true, accurate, and complete

James E. Rasmussen, Director of Environmental Assurance. Permits and Policy Division iName and official title of owner/operator OR owner/operator's authorized representative

\begin{tabular}{|c|c|c|}
\hline 0 & 5 & $\begin{array}{l}\text { Max Daily } \\
\text { Amount (codo) }\end{array}$ \\
\hline & & \\
\hline 0 & 5 & $\begin{array}{l}\text { Avg Daily } \\
\text { Anount }(c o d \theta)\end{array}$ \\
\hline & 6 & \\
\hline 3 & 6 & 5 on-site (days) \\
\hline
\end{tabular}

\begin{tabular}{|l|l|l|l|l|l|l|l|l}
\hline 0 & 5 & Amount \\
\hline
\end{tabular}

\begin{tabular}{|l|l|l|}
\hline 0 & 5 & Avg Dally \\
\hline
\end{tabular}

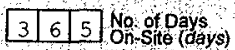
$015:$ Maxi Daily 015 Avg. Daily Avg. Daily Reactivity $\mathrm{X}$ Immediate (acute) Delayed (chronic)

\section{(3)} No. of Days

$\frac{1998}{\mathrm{~T}} \mathrm{~T}$ Chec

$\begin{array}{ccc}y & e \\ 0 & 0 & 0 \\ 0 & -1\end{array}$

\begin{tabular}{|l|l|l|}
\hline$L$ & 2 & 4 \\
\hline$L$ & 2 & 4 \\
\hline$L$ & 2 & 4 \\
\hline$I$ & 2 & 4 \\
\hline$I$ & 2 & 4 \\
\hline$I$ & 2 & 4 \\
\hline
\end{tabular}

$277 \mathrm{~W}$ SHOP

291B HAND \& FOOT COUNTER

CHANGE TRAILER AT 300-FF-1

305 TEST FACILITY CYLINDER DOCK $305 A$ SHOP

$306 \mathrm{E} \_\mathrm{LAB}$

Title SHIET COMMANDER $24 \mathrm{Hr}$. Phone $(509) \quad 373-3800$

Title 24 Hir. Phone 1 )

\begin{tabular}{llllll}
\hline & & 2 & 4 \\
\end{tabular}

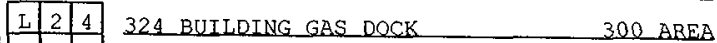
\begin{tabular}{lllll}
\hline & 2 & 4 & 325 BUIIDING ___ & 300 AREA
\end{tabular} \begin{tabular}{|l|l|l|l|l}
\hline & 2 & 4 & $327, \mathrm{LAB}$ \\
\hline & &
\end{tabular} 300 AREA \begin{tabular}{llll} 
I & 2 & 4 \\
\hline
\end{tabular} \begin{tabular}{lll}
$\mathrm{L}$ & 2 & 4 \\
\hline
\end{tabular} \begin{tabular}{|l|l|l|l|l|l|l}
\hline$L$ & 2 & 4 & 30 \\
\hline
\end{tabular} 300 ABEA \begin{tabular}{|l|l|l|l|l}
\hline $\mathrm{L}$ & 2 & 4 \\
\hline & & 3712 & STORAGE BUILDING & 300 AREA
\end{tabular}

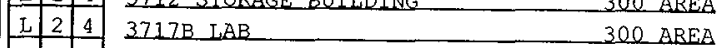

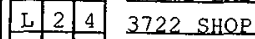
\begin{tabular}{llll}
$\mathrm{L}$ & 2 & 4 & 384 STEAM PLANT, DEACTIVATED \\
\hline
\end{tabular} 300 AREA \begin{tabular}{llll}
\hline$A$ & 2 & 7 \\
4
\end{tabular} 43 EUELS STORAGE FACILITY 300 AREA 400 AREA 400 AREA 


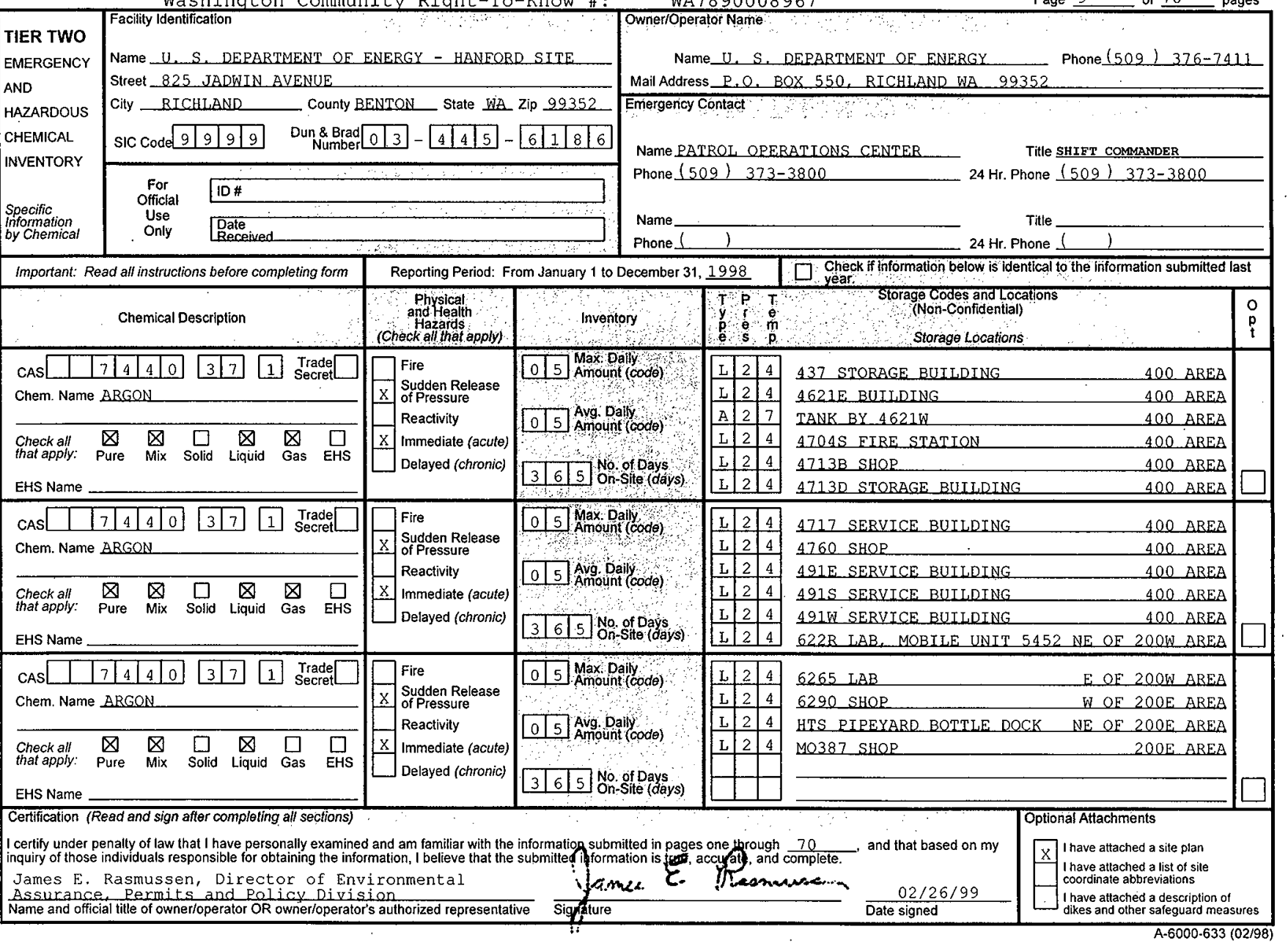


Washington Community Right-To-know \# : Facility Identification

Name U. S. DEPARTMENT OF ENERGY - HANEORD SITE Street 825 JADWIN AVENUE

City RICHLAND _ County BENTON _ State WA Zip 99352

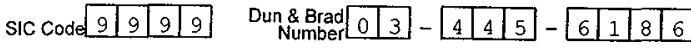

For
Official
Use
Only

WA7 890008967

Page 10 of 70

Phone (509) $376-7411$

Name_U, S. DERARTMENT OF ENERGY Mail Address_P.O_BOX 550, BICHLAND WR 99352 Emerigency Contä́ct:

Name PATROL OPERATIONS CENTER Phone $(509) \quad 373-3800$

Name

Phone 1
Title SHIET COMMANDER 24 Hr. Phone (509) 373-3800

Title by chemical

Reporting Period: From January 1 to December 31, 1998

Physical
and Heath
Hazards
(Chockall that apply)

Chemical Description (Chock all that apply) \begin{tabular}{l|l|l|l|l|l|l|l|l|l|}
\hline CAS & & & 1 & 3 & 0 & 2 \\
\hline
\end{tabular} Chem. Name BENTONITE

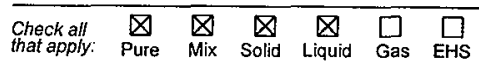
EHS Name CAS \begin{tabular}{l|l|l|l|l|l|l|l|l|}
\hline & & 1 & 3 & 0 & 2 \\
\hline
\end{tabular} Chem. Name BENTONITE

\begin{tabular}{lllllll}
\hline Checkall & $\bigotimes$ & $\bigotimes$ & $\bigotimes$ & $\bigotimes$ & $\square$ & $\square$
\end{tabular} that apply: Pure Mix Solid Liquid Gas EHS EHS Name

\begin{tabular}{|c|c|c|c|c|c|c|c|}
\hline CAS & & & & & & & \\
\hline
\end{tabular}
Chem. Name BRQMOTRIETUOROMETHANE (HALON 1301) $\begin{array}{llllll}\text { Check all } & \square & \square & \square & \square & \square\end{array}$ Mix Solid Liquid Gas EHS EHS Name Certification (Read and sign after completing all sections):

\section{Fire}

Sudden Release Reactivity $\mathrm{X}$ Delayed (chronic)

\section{Fire}

Sudden Release of Pressure

Reactivity Immediate (acute) $\mathrm{X}$ Delayed (chronic)

\section{Fire \\ $\mathrm{x}$ Sudden Release \\ $\mathrm{X}$ of Pressure \\ Reactivity \\ $\mathrm{x}$ Immediate (acute) Delayed (chronic)} of Pressure Immediate (acute)

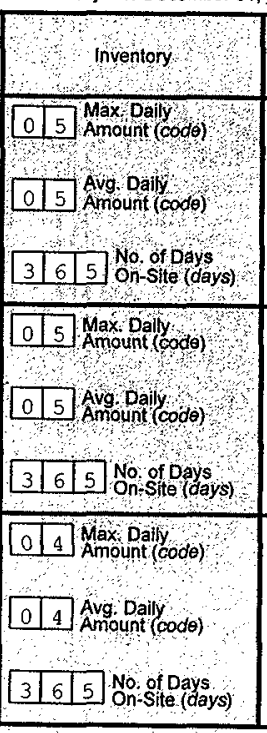

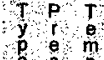

e $\quad$ s

\begin{tabular}{|l|l|l|}
\hline$E$ & 1 & 4 \\
\hline
\end{tabular}

F 114 CONEX BOX NE OF $105 \mathrm{~kW}$

E 114 SHED S OF 2025E

$M \quad 1 \quad 4 \quad 222 \mathrm{SA} \perp A B$

\begin{tabular}{llll}
$\mathrm{N}$ & 1 & 4 & $234-52$ BUILDING \\
\hline & 1 & 4
\end{tabular}

$277 \mathrm{~T}$ BUITDING

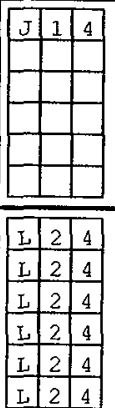

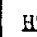

\section{$2101 M$ BUILDING}

HTS PIPEYARD WASTE PAD

NE OE 2OOE AREA

$100 \mathrm{~K}$ AREA I0OK AREA $200 E$ AREA 200W AREA 200W AREA 200W AREA

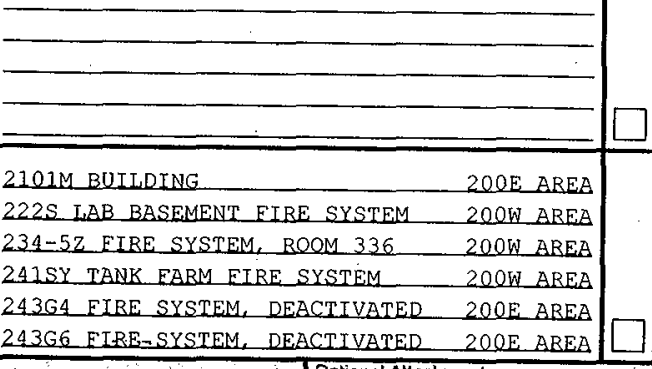

$200 E$ AREA 222S IAB BASEMENT FIRE SYSTEM 200 W AREA 200W AREA

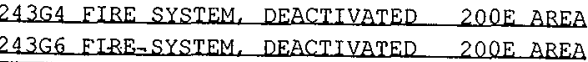
Optional Attachments
$\mathrm{X}$
$\begin{aligned} & \text { I have attached a site plan } \\ & \text { I have attached a list of site } \\ & \text { coordinate abbreviations } \\ & \text { I have attached a description of } \\ & \text { dikes and other safeguard measures }\end{aligned}$ A-6000-633 (02/98)

certify under penalty of law that 1 have personally examined and am familiar with the information submitted in pages one through 70 .
rnquiry of those individuals responsible for obtaining the information, I believe that the submitted in tormation is tryed on my rnquiry of those individuals responsible for obtaining the information, I believe
James E. Rasmussen, Director of Envi ronmental Assurance Permits and Poli Division Wame and official title of owner/operator OR owner/operator's authorized representative

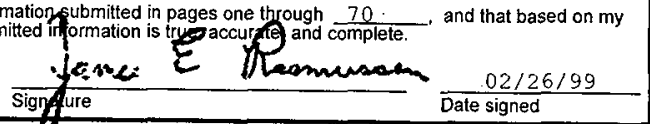
$\frac{.02 / 26}{\text { Date signed }}$ 
Washington Community Right-To-know \#: WA7 890008967

Page 11 of 70

TIER TWO

EMERGENCY

AND

HAZARDOUS

CHEMICAL

INVENTORY

Specific

by Chemical
Name U, S. DEPARTMENT OF ENERGY - HANEORD SITE

Street 825 JADFIN AVENUE,

City RICHIAND County BENTON State WA Zip 99352

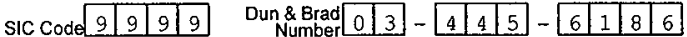

\begin{tabular}{|c|c|}
\hline $\begin{array}{c}\text { For } \\
\text { Official }\end{array}$ & ID \# \\
\hline $\begin{array}{l}\text { Use } \\
\text { Only }\end{array}$ & $\begin{array}{l}\text { Date } \\
\text { Received }\end{array}$ \\
\hline
\end{tabular}
Owner/Operator Name

Name $U$. S, DERARTMENT OE ENERGY Phone $(502) \quad 376-7411$ Mail Address_P.O. BOX 550, RICHLAND WA 99352 Emergency Contact

Name PATROL, OPERATIONS CENTER

Phone (509) 373-3800

Name

Phone ( )
Title SHIFT COMANDER $24 \mathrm{Hr}$. Phone (509) 373-3800

Title $24 \mathrm{Hr}$. Phone
Important: Read all instructions before completing form

Chemical Description

\section{Phisical and Health (Chock ail that apply)}

Reporting Period: From January 1 to December 31, 1998

CASL \begin{tabular}{|l|l|l|l|l|l|l|l|}
\hline & & & & 7 & 5 \\
\hline
\end{tabular} Chem. Name BROMOTRIELUOROMETHAN.

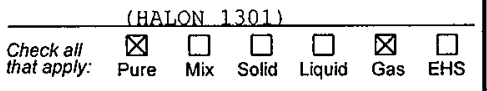

EHS Name

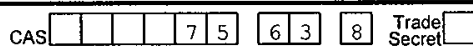

Chem. Name BROMOTRIELUOROMETHAN. (HALON 1301)

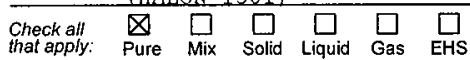

EHS Name

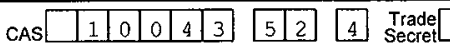

Chem. Name CAICEUM CHLORIDE

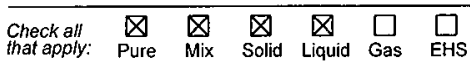

EHS Name

Certification (Read and sign after completing all sections)
Fire

$x$ Sudden Releas

Reactivity

$\mathrm{X}$ Immediate (acute)

Delayed (chronic)

\begin{tabular}{|c|c|}
\hline & $\mathrm{Fil}$ \\
\hline $\mathrm{X}$ & $\begin{array}{l}\text { Sudden Release } \\
\text { of Pressure }\end{array}$ \\
\hline & Reactivity \\
\hline $\mathrm{X}$ & Immediate (acute) \\
\hline & Delayed (chroni \\
\hline
\end{tabular}

Delayed (chronic)

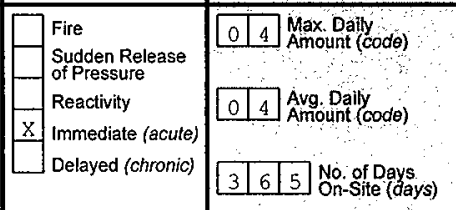

\begin{tabular}{|l|l|l}
\hline 0 & 4 & Avg baily \\
Amount (code)
\end{tabular}

3 ( 65 No of Days

(n)

- 4 Max Dally

Amount (code)

\begin{tabular}{lll}
\hline 0 & 4 & Avg Daily \\
Amount
\end{tabular}

Avg Daily

\begin{tabular}{l|l|l|l}
\hline 3 & 6 & 5 & No of Days \\
On-Site (days)
\end{tabular}
Ínventory

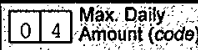

$\begin{array}{lll}\mathbf{T} & \mathbf{T} \\ \mathbf{p} & \mathbf{e} & \mathrm{e} \\ \mathrm{p} & \mathrm{p}\end{array}$

s $p$

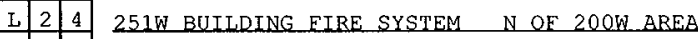
\begin{tabular}{llll}
\hline$L$ & 2 & 4 & $27012 A$ \\
$2 U$
\end{tabular} $\begin{array}{llllll}L & 2 & 4 & 2704 \\ 2 & & \end{array}$

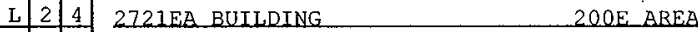

\begin{tabular}{l|l|l|l|l}
\hline $\mathrm{L}$ & 2 & 4 \\
\hline
\end{tabular}

\begin{tabular}{lll}
\hline $\mathrm{L}$ & 2 & 4 \\
\hline
\end{tabular} 403 EIRE SYSTEM, ROOM $900 \quad 400$ AREA

\begin{tabular}{|l|l|l|l|l|l|l}
\hline L & 2 & 4 \\
\hline
\end{tabular}

\begin{tabular}{lll} 
L & 2 & 4 \\
\hline
\end{tabular} 4703 BUILDING EIRE SYSTEM

\begin{tabular}{lll|l}
\hline L & 2 & 4 \\
\hline
\end{tabular} 4721_BUILDING EIRE SYSTEM

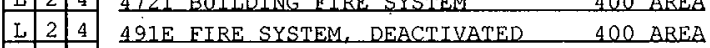

400 AREA

609C BLDG SYSTEM RT 3 BETWEEN 200E/W_ABEAS

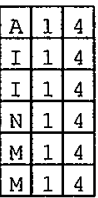

100-DR-1 ORERABLE UNIT

$1171 \mathrm{C}$ STORAGE SHED, DEACTIVATED 1100 AREA

$1724 \mathrm{KA}$ EOUIPMENT SHED

222SA LAB

STORAGE UNIT BY 234-52

$234-52$ BUILDING
100D/DR_AREA
D 1100 AREA

$100 K$ AREA

200W ABEA 200W AREA $200 W$ AREA
I certify under penalty of law that I have personally examined and am familiar with the information submitted in pages one through 70

inquiry of those individuals responsible for obtaining the information, I believe that

Assurance, Permits and Policy Division

Name and official title of owner/operator OR owner/operator's authorized representative

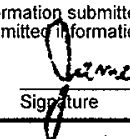

and that based on my

$02 / 26 / 99$ Date signed

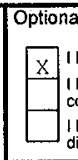




\section{TIER TWO}

EMERGENCY AND

HAZARDOUS

CHEMICAL

INVENTORY

Specific
Information
by Chemical
Name U. S. DEPARTMENT OF ENERGY - HANEORD SITE

Street 825 JADWIN AVENUE

City _RICHLAND _ County BENTON_ State WA Zip 99352

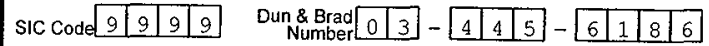

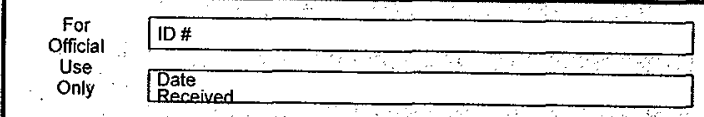

\section{Ownertoperator Name}

Name U. S. DEPARTMENT OF ENERGY Mail Address _. O. BOX 550 RICHLAND WA 99352 Phone (509) $376-7411$ Emergency Contact

Name PATROL OPERATIONS CENTER

Phone (509) 373-3800

Title SHIET COMMANDER $24 \mathrm{Hr}$. Phone (509) 373-3800

Name

Phone (1)

Title $24 \mathrm{Hr}$. Phane ( )

Important: Read all instructions before completing form Chemical Description

Physical
and Health
Aazaids
(Check all that apply)

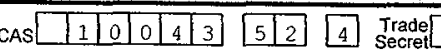
Chem. Name CALCIUM CULROIDE $\begin{array}{lccccc}\text { Check all } & \underset{\text { that apply: }}{\otimes} & \underset{\text { Mix }}{\otimes} & \underset{\text { Solid }}{\otimes} & \underset{\text { Liquid }}{\otimes} & \square \\ \text { Gas } & \square\end{array}$ EHS Name cas \begin{tabular}{l|l|l|l|l|l|l|l|}
\hline & & 7 & 4 & 4 & 0 \\
\hline 4 & 4 \\
\hline
\end{tabular} Chem. Name CARBON

\begin{tabular}{|l|l|} 
& $\begin{array}{l}\text { Fire } \\
\text { Sudden Release } \\
\text { of Pressure } \\
\text { Reactivity } \\
\text { Immediate (acute) } \\
\text { Immelayed (chronic) } \\
\text { Delaye }\end{array}$ \\
\hline
\end{tabular}

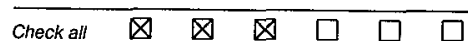
that apply: Pure Mix Solid Liquid Gas EHS EHS Name

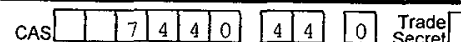
Chem. Name CARBON

\begin{tabular}{|c|c|c|c|c|c|c|}
\hline $\begin{array}{l}\text { Check all } \\
\text { that apply: }\end{array}$ & $\underset{\text { Pure }}{\bigotimes}$ & $\underset{\text { Mix }}{\bigotimes}$ & $\underset{\text { Solid }}{\bigotimes}$ & $\square_{\text {Liquid }}^{\square}$ & $\square$ & $\square_{\text {EHS }}$ \\
\hline
\end{tabular}

\begin{tabular}{|l|l}
$\mathrm{x}$ & $\begin{array}{l}\text { Fire } \\
\text { Sudden Release } \\
\text { of Pressure } \\
\text { Reactivity } \\
\text { Immediate (acute) } \\
\text { Delayed (chronic) }\end{array}$ \\
\hline
\end{tabular}

Certification (Read and sign after completing all sections)

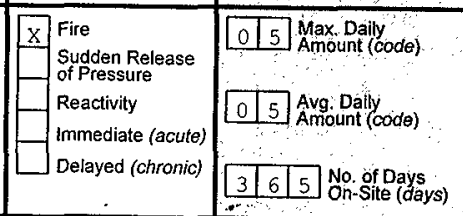

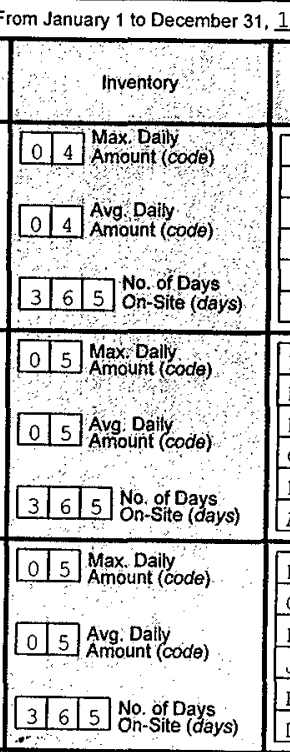

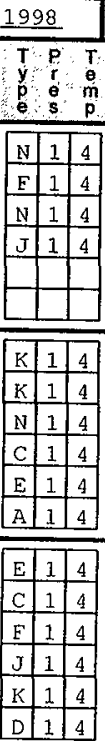
Check
year.

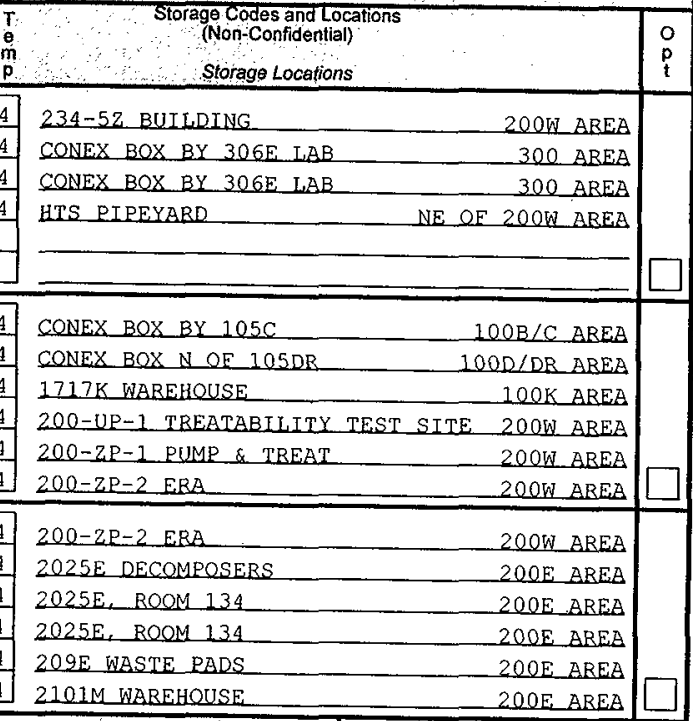

Optional Attachments I certify under penalty of law that I have personally examined and am familiar with the information submitted in pages one through 70
mquiry of those individuals responsible for obtaining the information, I believe that the submitteghinformation is true, accurate, and compleie.

- James E. Rasmussen, Director of Environmental Assurance, Permits and Policy Division Name and official title of owner/operator OR owner/operator's authorized representative

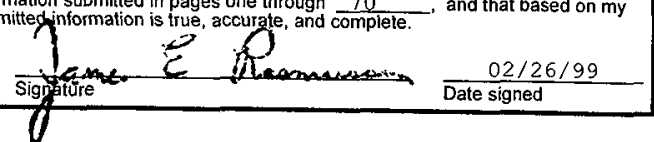
coordinate abbreviations 


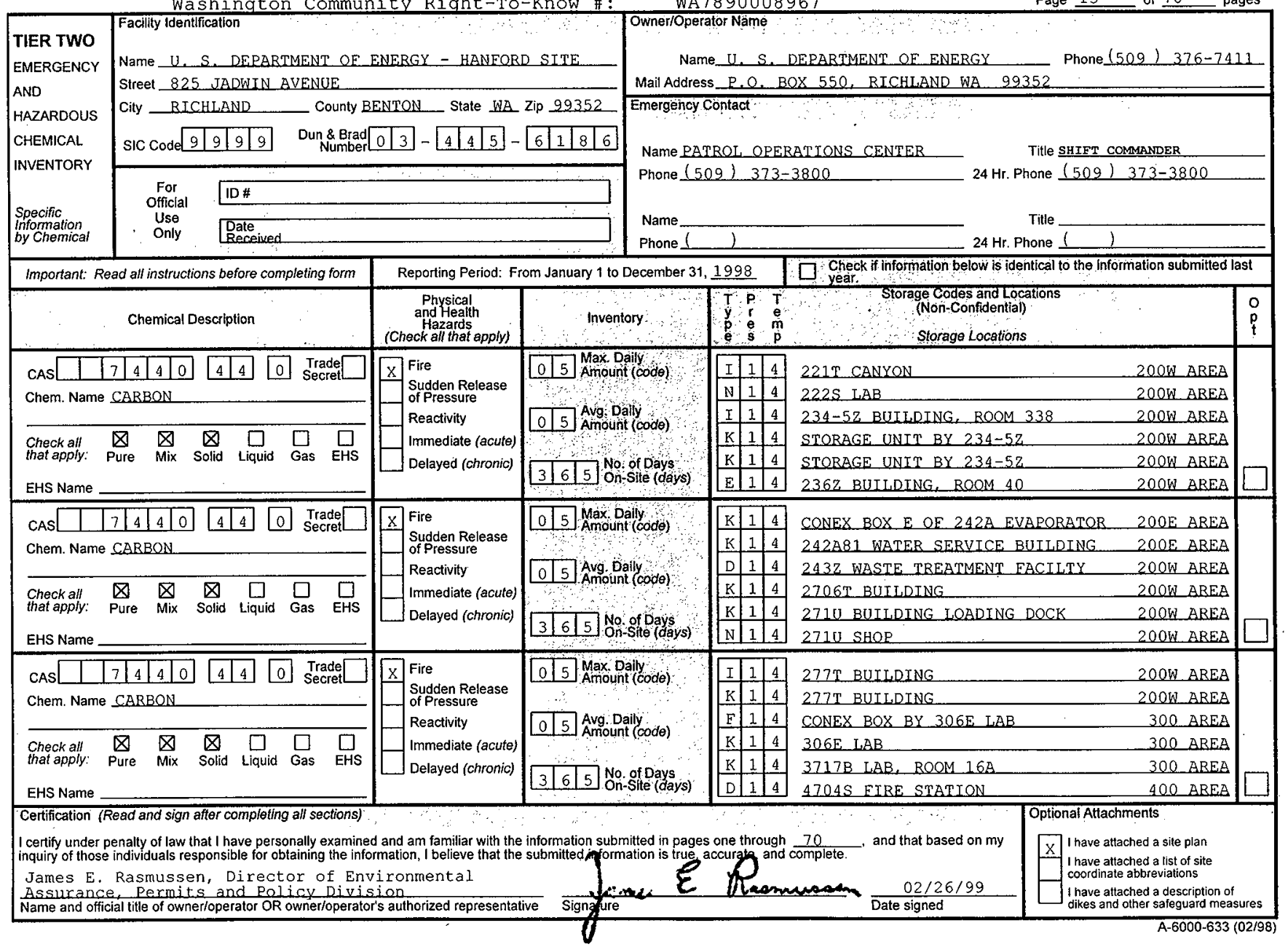




\begin{tabular}{|c|c|}
\hline TIER TWO & Facility Identification \\
\hline EMERGENCY & Name U. S. DERARTMENT OF ENERGY - HANFORD SITE \\
\hline $\begin{array}{l}\text { AND } \\
\text { HAZARDOUS }\end{array}$ & $\begin{array}{l}\text { Street } 825 \text { JADWIN AVENUE } \\
\text { City RICHLAND_ County BENTON State WA Zip } 99352\end{array}$ \\
\hline CHEMICAL & \begin{tabular}{|l|l|l|l|l|} 
SIC code & 9 & 9 & 9 & 9 \\
\end{tabular} \begin{tabular}{|l|l|l|l|l|l|} 
Dun \& Brad \\
Number
\end{tabular} \\
\hline & $\begin{array}{l}\text { For } \\
\text { Official }\end{array}$ \\
\hline $\begin{array}{l}\text { Information } \\
\text { by Chemical }\end{array}$ & $\begin{array}{l}\text { Use } \\
\text { Only }\end{array}$ \\
\hline
\end{tabular}

Name PATROL OPERATIONS CENTER Phone $(509) \quad 373^{\circ}-3800$

Name

Phone 1
Title SHIFT COMMANDER $24 \mathrm{Hr}$. Phone $(509) 373-3800$

Title
Important: Read all instructions before completing form

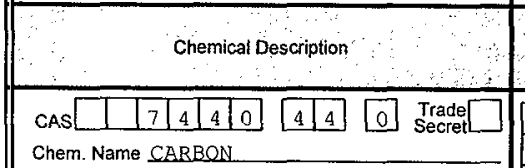

Chem. Name CARBON

Reporting Period: From January 1 to Décember 31, 1998

\section{and Health} and Health (Check all that apply)

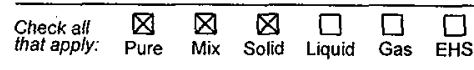

EHS Name

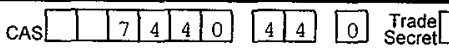
Chem. Name $C A R B O N$

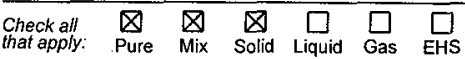
EHS Name

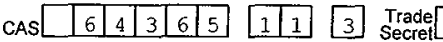
Chem. Name CHARCOAL

\begin{tabular}{lllllll}
\hline Check all & $\square$ & $\square$ & $\square$ & $\square$ & $\square$ & $\square$
\end{tabular} that apply: Pure Mix Solid Liquid Gas EHS

EHS Name

Certification (Read and sign after completing all sections) \begin{tabular}{|l|l}
$\mathrm{X}$ & $\begin{array}{l}\text { Fire } \\
\text { Sudden Release } \\
\text { of Pressure }\end{array}$ \\
\hline
\end{tabular}

Reactivity

Immediate (acute)

Delayed (chronic)

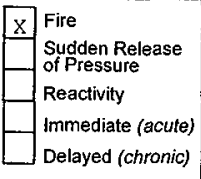

\section{$x$ Fire}

Sudden Release

of Pressure

Reactivity

Immediate (acute)

$x$ Delayed (chronic)
II certify under penalty of law that I have personally examined and am familiar with the information submitted in pages one through 70 inquiry of those individuals responsible for obtaining the information, I believe that the submitted information is true, accurate, and complete

James E. Rasmussen, Director of Environmental Assurance, Permits and Policy Division iName and official title of owner/operator OR owner/operator's authorized representative

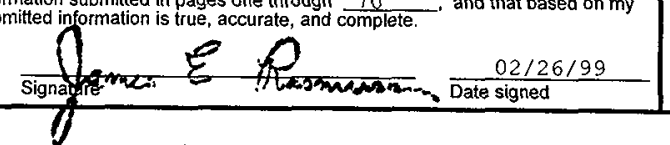
Check if information below is identical to the information submitted last

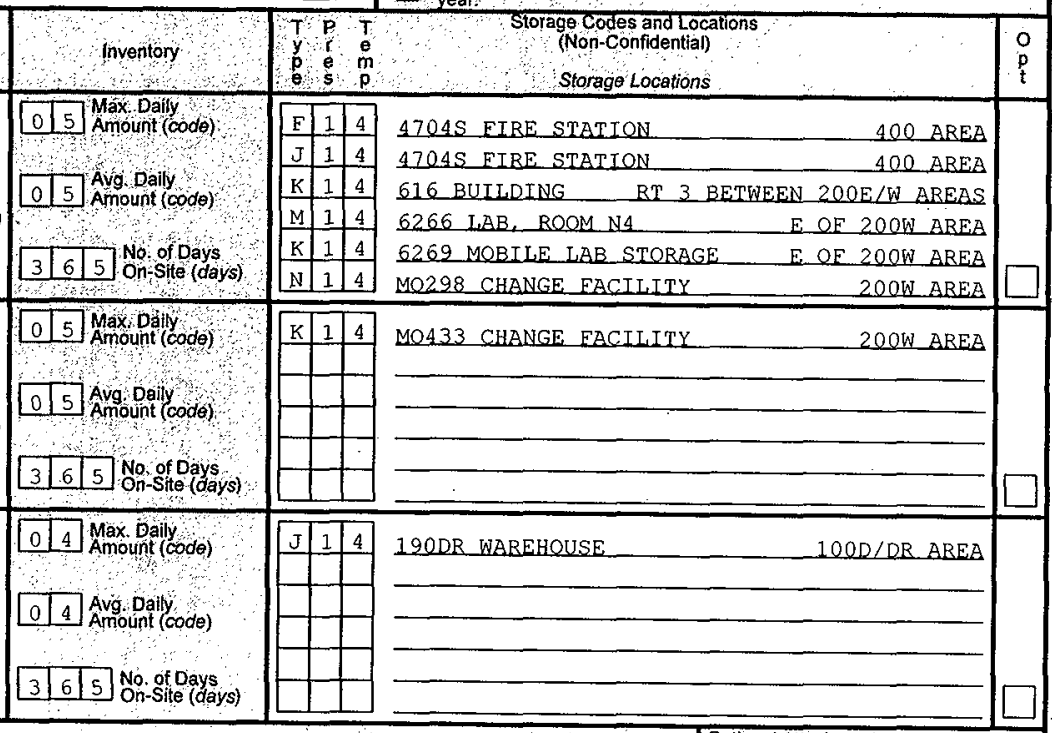

Optional Attachments

$x$ I have attached a site plan I have attached a list of site coordinate abbreviations

I have attached a description of dikes and other safeguard measures A-6000-633 (02/98) 


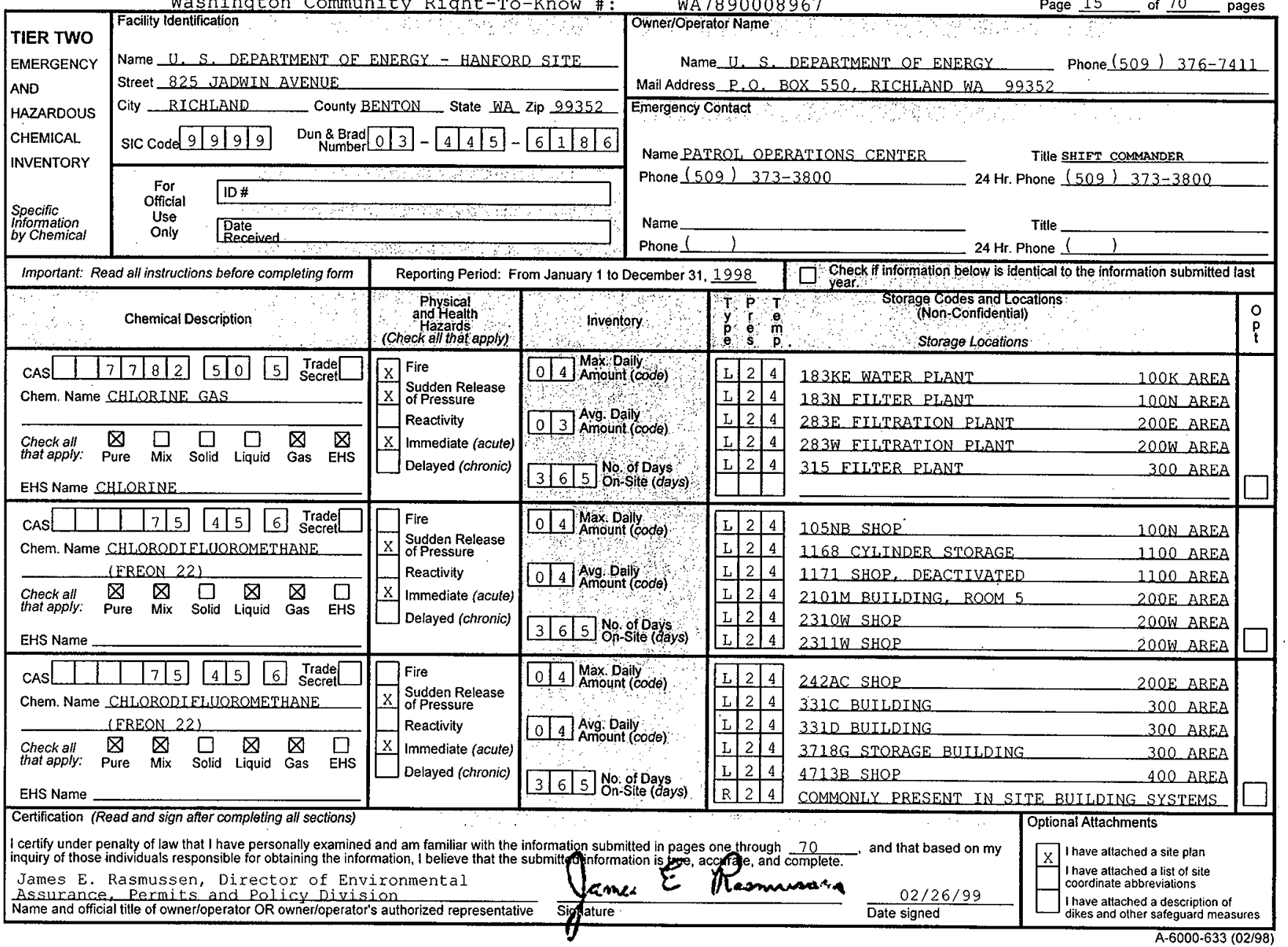




\section{TIER TWO}

EMERGENCY AND

HAZARDOUS

CHEMICAL

INVENTORY

Specific

Name U. S. DEPARTMENT OF ENERGY - HANEORO SITE

Street 825 JADWIN AVENUE

City BICHLAND County BENTON State WA Zip 99352

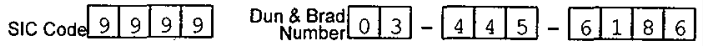

$\begin{array}{lll}\text { For } & \\ \text { Official } & \\ \text { Use. } \\ \text { Only }\end{array}$

Owner/Operator Name

Name U. S. DERARTMENT OF ENERGY Mail Address P.O. BOX 550 , RICHLAND WA 99352

Phone $(502) \quad 376-7411$
Name PATROL OPERATIONS CENTER Phone $(509) \quad 373-3800$

Name

Phone
Reporting Period: From January 1 to December 31. 1998

\section{Physical
and Health} and azards

Chemical Description

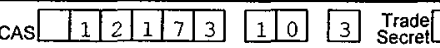

Chem. Name CLINOPTHOLITE

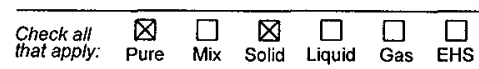

EHS Name

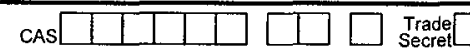

Chem. Name COAL

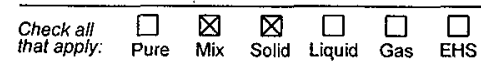

EHS Name

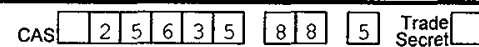
Chem. Name COMPRESSED AIR $\begin{array}{lccccc}\text { Check all } & \square & \square & \square & \square & \square \\ \text { that apply: } & \square & \square \\ \text { Pure }\end{array}$

EHS Name

Certification (Read and sign after compleling all sectionș) (Chock all that apply)

\section{$\square$ Fire}

Sudden Release

of Pressure

Reactivity

$\square$ Immediate (acute)

$x$ Delayed (chronic)

\begin{tabular}{|l|l|}
\hline$x$ & $\begin{array}{l}\text { Fire } \\
\text { Sudden Release } \\
\text { of Pressure } \\
\text { Reactivity } \\
\text { Immediate (acute) } \\
\text { Immede } \\
\text { Delayed (chronic) }\end{array}$ \\
\hline
\end{tabular}

\section{$\square$ Fire}

$\mathrm{X}$ Sudden Release

Reactivity

Immediate (acute)

Delayed (chronic)

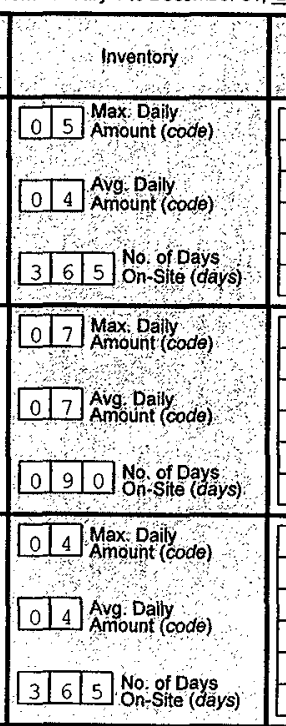
.

I certify under penalty of law that I have personaliy examined and am familiar with the information submitted in pages one through 70 i inquiry of those individuals responsible for obtaining the information, I believe that the submilted submitted in pages one through 70 James E. Rasmussen, Director of Environmental
Assurance, Permits and Policy Division Name and official title of owner/operator OR owner/operator's authorized representative

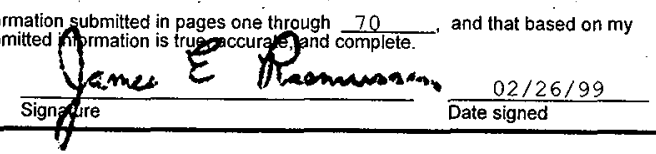

Title SHIFT COMMANDER 24 Hr. Phone $(509) 373-3800$ $24 \mathrm{Hr}$. Phone 1 ratrien Check if information below is identical to the information submitted last

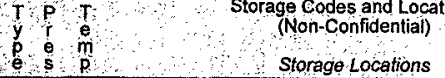

\begin{tabular}{|l|l|l|}
\hline$J$ & 1 & 4 \\
\hline$J$ & 1 & 4 \\
\hline & & \\
\hline & & \\
\hline & & \\
\hline & & \\
\hline$R$ & 1 & 4 \\
\hline & & \\
\hline & & \\
\hline & & \\
\hline & & \\
\hline & & \\
\hline
\end{tabular}

$1723 \mathrm{~N}$ WAREHOUSE

2101M WAREHOUSE $100 N$ AREA 200E AREA

\begin{tabular}{l|l|l|l}
\hline L & 2 & 4 & 1168 CYLINDER STORAGE
\end{tabular} \begin{tabular}{llll}
\hline & 2 & 4 \\
1171, SHOP DEACTIVATED
\end{tabular} 1100 AREA

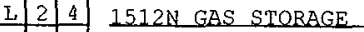
\begin{tabular}{llll}
\hline & 2 & 4 & \\
\hline & $1724 \mathrm{~KB}$ GAS STORAGE
\end{tabular}

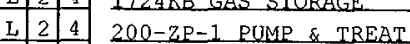
$100 \mathrm{~N}$ AREA $100 \mathrm{~K} A \mathrm{BEEA}$ 200W AREA 200W AREA

\section{Optional Aftachments}


Washington Community Right-To-know \#:

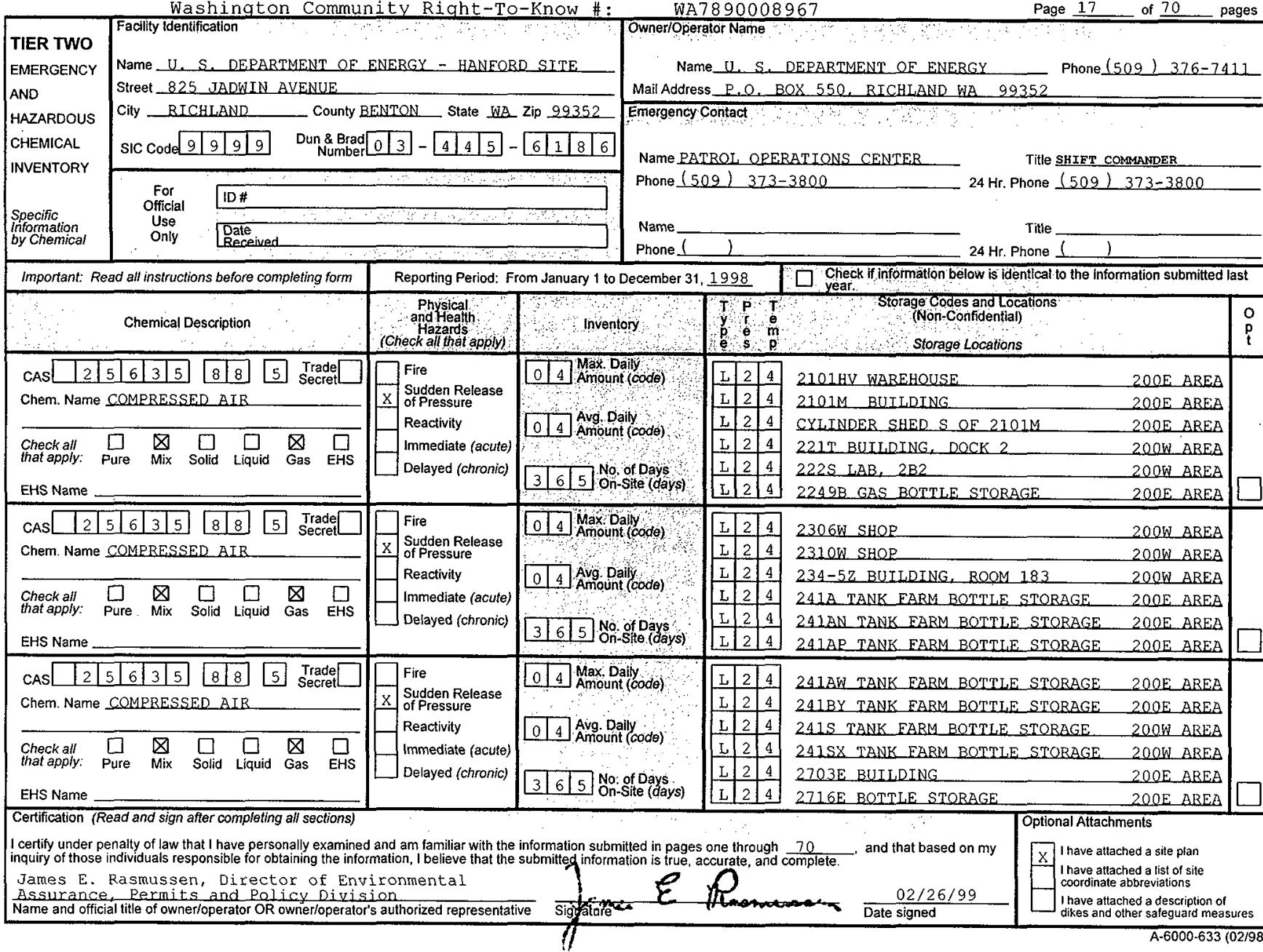




\section{TIER TWO}

EMERGENCY

AND

HAZARDOUS

CHEMICAL

INVENTORY

Specific
Information
by Chemical

Name U. S. DERARTMENT OE ENERGY - HANFORD SITE

Street 825 JADWIN AVENUE

City BICHLAND C County BENTON State WA Zip 99352

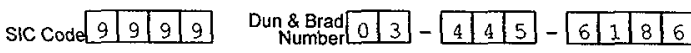

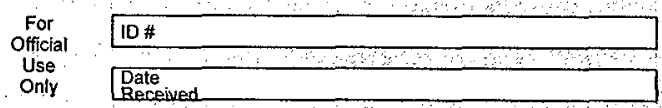

\section{Ownér/Operator Name}

Name_U, S, DEPARTMENT OF ENERGY Mail Address P.O. BOX 550, RICHLAND WA 99352 Emergency Contact

Name PATROL, OPERATIONS CENTER

Phone (509) $373-3800$

Name

Phone l
Phone $(509) \quad 376-7411$

Title SHIFT COMMANDER $24 \mathrm{Hr}$. Phone $(509) \quad 373-3800$

Title $24 \mathrm{Hr}$. Phone ( )
Important: Read all instructions before completing form Chemical Description CAS \begin{tabular}{|l|l|l|l|l|l|l|}
\hline & 2 & 5 & 6 & 3 & 5 \\
\hline 8 & 8 \\
\hline 5
\end{tabular}$\quad \begin{aligned} & \text { Trade } \\
& \text { Secret }\end{aligned}$ Chem. Name COMPRESSED AIR

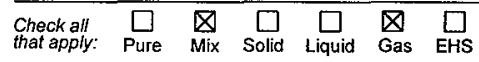
EHS Name \begin{tabular}{l|l|l|l|l|l|l|l|l|}
\hline CAS & 2 & 5 & 6 & 3 & 5 \\
\hline
\end{tabular} Chem. Name COMPRESSED AIR $\begin{array}{llll}\text { Checkall } & \square & \square & \square \\ \text { that apply: } & \square & \square & \square \\ \text { Pure } & \square\end{array}$ EHS Name

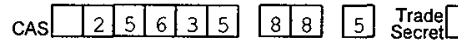
Chem. Name COMPRESSED BIR

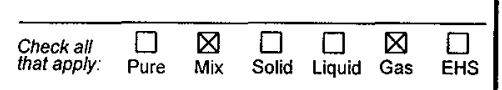

EHS Name

'Cerlfication (Read and sign after completing all sections) inquiry of those individual formation submitted in pages one inquiry of those individuals responsible for obtaining the information, I believe
James $E$. Rasmussen, Di rector of Environmental Assurance, Permits and Policy Division Name and official title of owner/operator OR owner/operator's authorized representative

\section{Physical Hazaids (Chock all that apply)}

Reporting Period: From January 1 to December 31, 1998

\begin{tabular}{|l|l}
\hline $\mathrm{F}$ & Fire \\
Sudden Release \\
of Pressure \\
Reactivity \\
Immediate (acute) \\
Delayed (chronic)
\end{tabular}

\section{Fire}

$\mathrm{X}$ Sudden Release $\mathrm{X}$ of Pressure Reactivity $\square$ Immediate (acute) Delayed (chronic)

\section{Fire} $\mathrm{X}$ Sudden Release $X$ of Pressure Reactivity - Immediate (acute) Delayed (chronic)

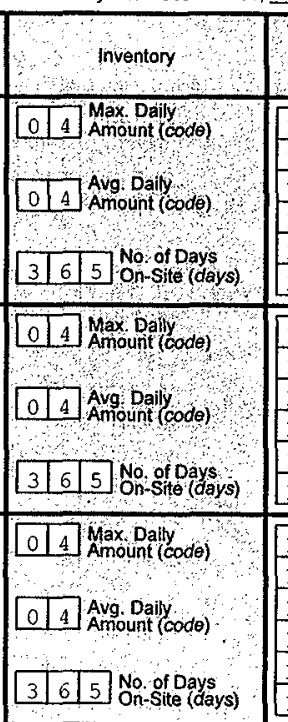

$T$
$T$
$T$

p o m

\begin{tabular}{|l|l|l|}
\hline$L$ & 2 & 4 \\
\hline
\end{tabular}

\begin{tabular}{|l|l|l|}
\hline $\mathrm{L}$ & 2 & 4 \\
\hline $\mathrm{L}$ & 2 & 4 \\
\hline
\end{tabular}

\begin{tabular}{|l|l|l|}
\hline $\mathrm{L}$ & 2 & 4 \\
\hline
\end{tabular}

\begin{tabular}{|l|l|l|}
\hline & 2 & 4 \\
\hline $\mathrm{L}$ & 2 & 4 \\
\hline
\end{tabular}

\begin{tabular}{|l|l|l|}
\hline $\mathrm{I}$ & 2 & 4 \\
\hline
\end{tabular}

\begin{tabular}{l|l|l|}
\hline & 2 & 4 \\
\hline L & 2 & 4 \\
\hline
\end{tabular}

\begin{tabular}{|l|l|l|}
\hline $\mathrm{L}$ & 2 & 4 \\
\hline
\end{tabular}

\begin{tabular}{|l|l|l|}
\hline $\mathrm{L}$ & 2 & 4 \\
\hline $\mathrm{I}$ & 2 & 4 \\
\hline
\end{tabular}

\begin{tabular}{l|l|l}
$\mathrm{L}$ & 2 & 4 \\
\hline
\end{tabular}

\begin{tabular}{l|l|l|}
\hline $\mathrm{L}$ & 2 & 4 \\
\hline
\end{tabular}

\begin{tabular}{|l|l|l|}
\hline$L$ & 2 & 4 \\
\hline
\end{tabular}

\begin{tabular}{|l|l|l|}
\hline$L$ & 2 & 4 \\
\hline
\end{tabular}

\section{\begin{tabular}{|l|l|l|}
\hline $\mathrm{L}$ & 2 & 4 \\
\hline
\end{tabular}}

\begin{tabular}{|l|l|l}
\hline $\mathrm{L}$ & 2 & 4 \\
\hline & &
\end{tabular}

408A_EAST HEAT DUMP

$\begin{array}{lllll}\mathrm{L} & 2 & 4 & 408 \mathrm{~B} \text { SOUTH HEAT DUMP }\end{array}$

\begin{tabular}{lll}
$\mathrm{L}$ & 2 & 4 \\
\hline
\end{tabular}

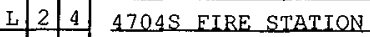

$4713 B$ SHOP

$271 B$ BUILDING 2721EA BUILDING. $27342 B$ GAS STORAGE 277A SHOP_BOTTIE DOCK

305 TEST EACILITY

BONEYARD BY $306 \mathrm{E}$ $306 \mathrm{E}$ I A AB

328 SHOP 3709 A EIRE STATION Check $200 E$ AREA $200 E$ AREA 200E AREA $200 E$ AREA 300 AREA 300 AREA 300 AREA 300 AREA 300 AREA 300 AREA

405 REACTOR BUILDING 400 AREA 400 AREA 400 AREA 400 AREA 400 AREA 400 AREA Optional Attachments and that based on my $X$ I have attached a site plan I have attached a list of site coordinate aboreviations I have attached a description of dikes and other safeguard measures 


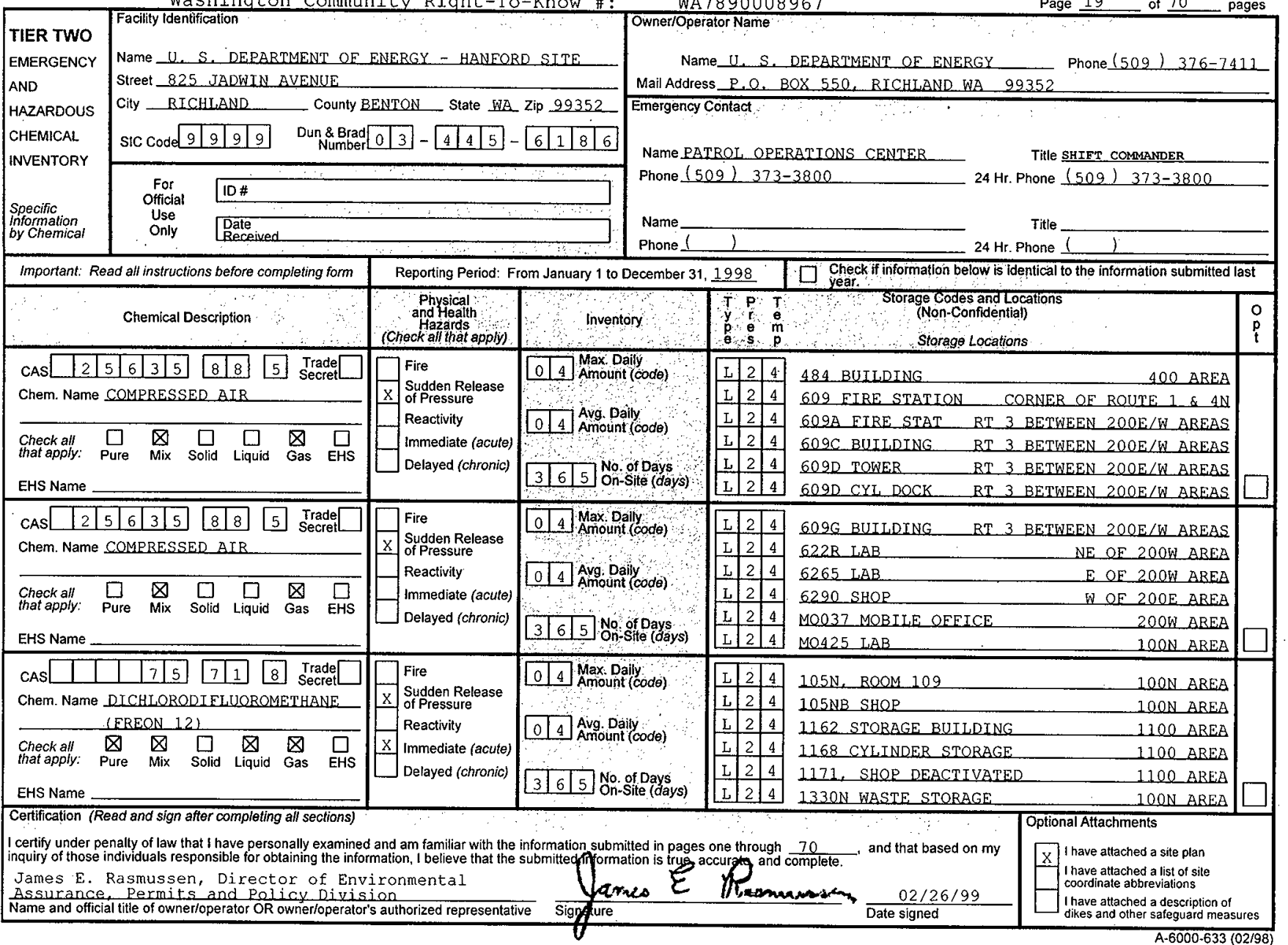




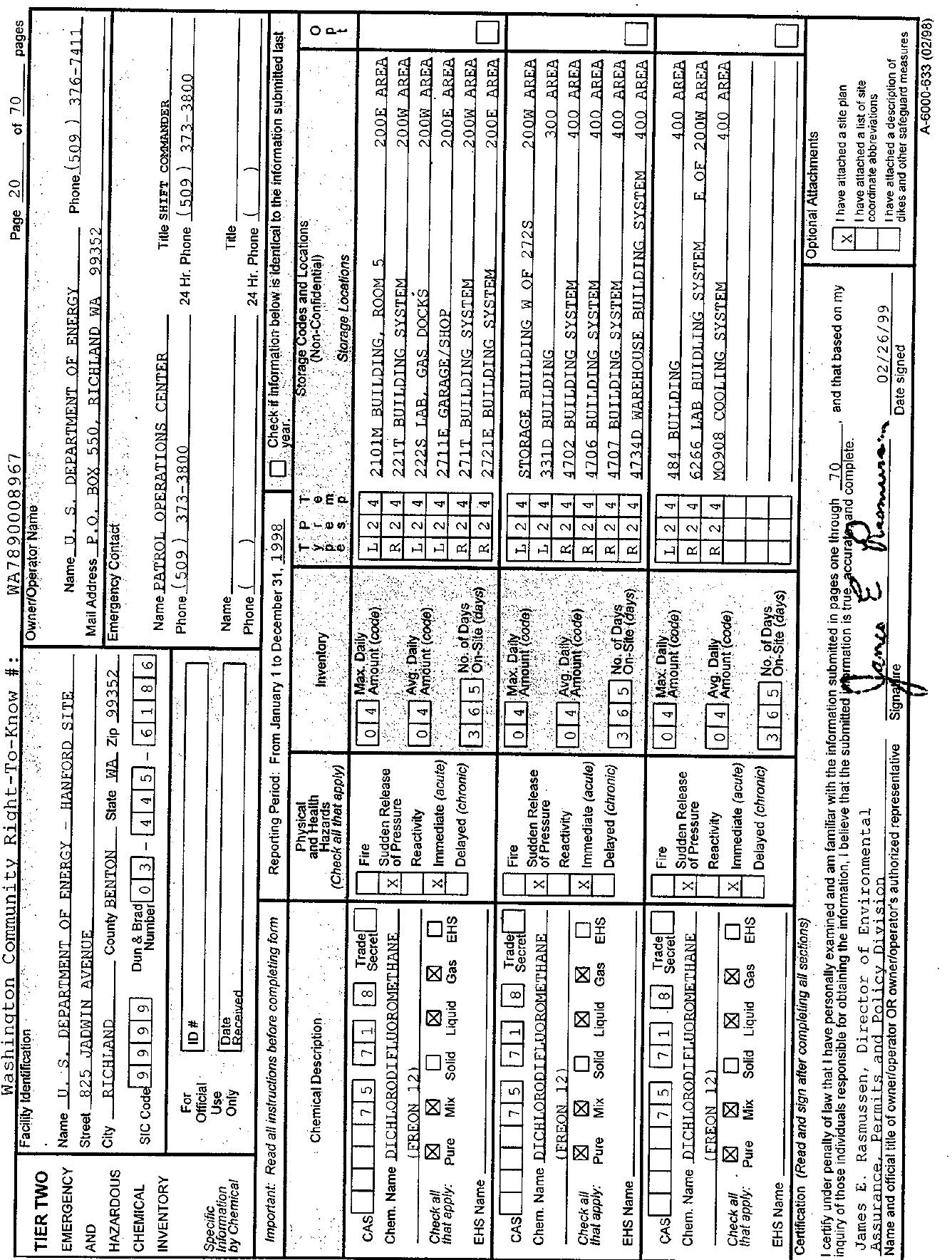


Washington Community Right-To-know \#:

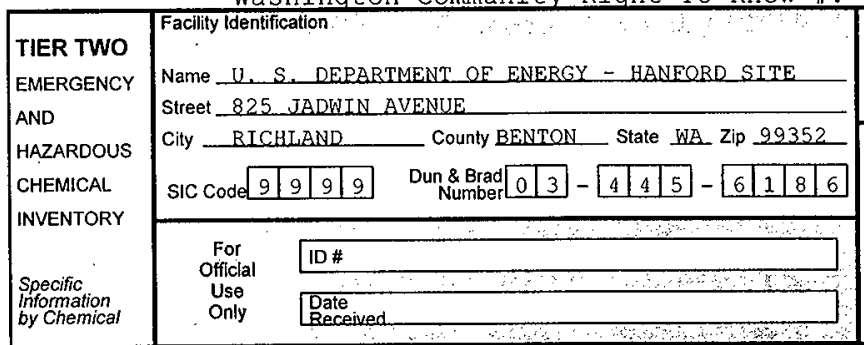

WA7890008967

Page 21 of 70 Owner/Operator Name

Name_U. $S$, DEPARTMENT OF ENERGY Phone $(509) \quad 376-7411$ Mail Address _R.O._BOX 550, RICHLAND WA 99352 Emergency Contact

Name PATROI OPERATIONS CENTER Phone (509) 373-3800

Name

Phone (... )

Reporting Period: From January 1 to December 31,
Physical
and Health
Hazards
(Check all that apply)

$\mathrm{X}$ Fire

Sudden Release of Pressure

Reactivity $\mathrm{X}$ Immediate (acute) $x$ Delayed (chronic)

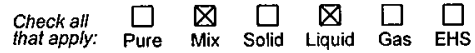
EHS Name CAS \begin{tabular}{|l|l|l|l|l|l|l|}
\hline & & & & & \\
\hline
\end{tabular} Chem. Name DIESEL FUELS, GRADE $1 \& 2$ $\begin{array}{lllll}\text { Checkall } & \square & \square & \square & \square \\ \text { that apply: } & \square & \square & \square \\ \text { Pure }\end{array}$ EHS Name CAS \begin{tabular}{l|l|l|l|l|l|l|l|}
\hline & & & & \\
\hline
\end{tabular} Chem. Name DIESEL EUELS, GRADE 1 \& 2 Check all $\square \quad \square \quad \square \quad \square \quad \square \quad \square$ that apply: Pure Mix Solid Liquid Gas EHS EHS Name \begin{tabular}{|l|l|}
\hline $\mathrm{x}$ & $\begin{array}{l}\text { Fire } \\
\text { Sudden Release } \\
\text { of Pressure } \\
\\
\text { Reactivity }\end{array}$ \\
\hline $\mathrm{x}$ & $\begin{array}{l}\text { Immediate (acute) } \\
\mathrm{X}\end{array}$ \\
Delayed (chronic)
\end{tabular} Fire
Sudden Release of Pressure Reactivity $x$ immediate (acute) $x$ Delayed (chronic)

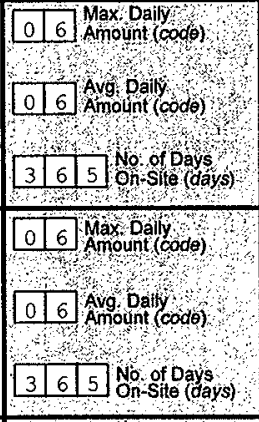

\begin{tabular}{|l|l|l}
\hline 0 & 6 \\
\hline
\end{tabular} Amoun Daily (code)

0 Avg Dally Avg; Dally \begin{tabular}{lll}
\hline 3 & 6 & 5
\end{tabular} No of Days

\begin{tabular}{|c|c|c|c|}
\hline p & $\theta$ & $\underset{\mathrm{p}}{\mathrm{e}}$ & $\begin{array}{l}\text { Storage Codes andLoc } \\
\text { (Non Confidential } \\
\text { Storage Locations }\end{array}$ \\
\hline$\underline{P}$ & 1 & 4 & 100-DR-1 ORERABLE UNI \\
\hline A. & 1 & 4 & 100N EOUIPMENT POOI, \\
\hline A & 1 & 4 & 1171 SHOP, DEACTIVATEI \\
\hline $\mathrm{C}$ & 1 & 4 & 1171 SHOP, DEACTIVATED \\
\hline A & 1 & 4 & TANK BY $182 \mathrm{~B}$ PUMPHOUSE \\
\hline A & 1 & 4 & 200-ZP-1 PUMP \& TREAT \\
\hline$B$ & 1 & 4 & UNDERGBQUND TANK BY 20 \\
\hline A & 1 & 4 & 212H CANTSTER STORAGE \\
\hline A & 1 & 4 & TANK NE OE 234-52. \\
\hline $\mathrm{B}$ & 1 & 4 & UNDERGROUND TANK BY 2 \\
\hline A & 1 & 4 & 251W_SWITCHING STATIO \\
\hline C & 1 & 4 & $2721 \mathrm{E}$ BUILDING \\
\hline B & 1 & 4 & UNDERGROUND TANK SE OF \\
\hline B & 1 & 4 & UNDERGROUND TANK SE OF \\
\hline A & 1 & 4 & TANK BY $282 B$ PUMP HOUS \\
\hline A & 1 & 4 & TANK BY 282BA PUMP HOU \\
\hline A & 1 & 4 & TANK E OE 284W DEACTIV \\
\hline$A$ & 1 & 4 & TANK W OF $300-E F-1$ OP \\
\hline & & & and that based on my \\
\hline
\end{tabular}
Date signed
Title SHIF'T COMANDER $24 \mathrm{Hr}$. Phone (509) 373-3800

Title

$24 \mathrm{Hr}$. Phone ( )
Cerfification (Read and sign after completing all sections)

I certify under penalty of law that I have personally examined and am familiar with the information submitted in inquiry of those individuals responsible for obtaining the information, I believe that
James E. Rasmussen, Di rector of Environmental

Assurance, Permits and Policy Division

Assurance, Permits and Po and official title of owner/operator OR owner/operator's authorized representative

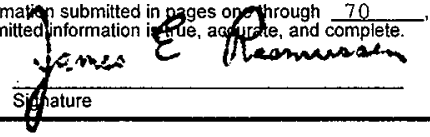

\section{$=$}




\begin{tabular}{|c|c|}
\hline \multirow{5}{*}{$\begin{array}{l}\text { TIER TWO } \\
\text { EMERGENCY } \\
\text { AND } \\
\text { HAZARDOUS } \\
\text { CHEMICAL } \\
\end{array}$} & Facility identification \\
\hline & Name $U$. S. DEPARTMENT OE ENERGY - HANEORD SITE, \\
\hline & ADWIN AVENUE \\
\hline & _ County BENTON_ State WA Zip 99352 \\
\hline & 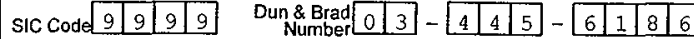 \\
\hline & For \\
\hline $\begin{array}{l}\text { Specific } \\
\text { information } \\
\text { by Chemical }\end{array}$ & $\begin{array}{l}\text { Use } \\
\text { Only }\end{array}$ \\
\hline
\end{tabular}

Name PATBOI_ OPERATIONS CENTER

Phone $(509) \quad 373-3800$

Title SHIFT COMMANDER

Name Phone (1) $24 \mathrm{Hr}$. Phone
Important: Read all instructions before completing form Chemical Description

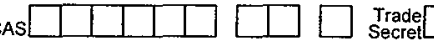
Chem Name DIESEI FUELS, GRADE $1 \& 2$ $\begin{array}{lllll}\text { Checkall } & \square & \square & \square & \square \\ \text { that apply: } & \square & \square & \square \\ \text { Pure } & \square\end{array}$ EHS Name

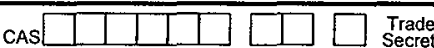
Chem. Name DIESEL FUELS, GRAOE $1 \& 2$ $\begin{array}{lllll}\text { Check all } & \square & \square & \square & \square \\ \text { that apply: } & \square & \square & \square \\ \text { Pure } & \square\end{array}$ EHS Name

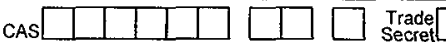
Chem Name DIESEI, FUELS, GRADE $1 \& 2$ Check all $\square \quad \square \quad \square \quad \square \quad \square \quad \square$ that apply: Pure Mix Solid Liquid Gas EHS

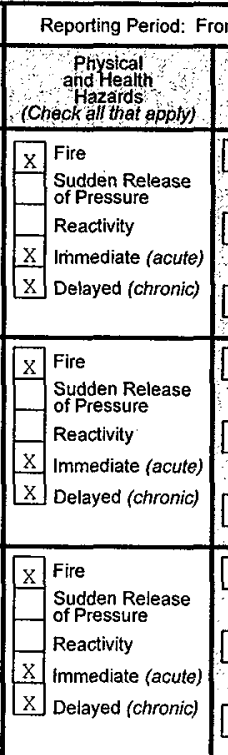

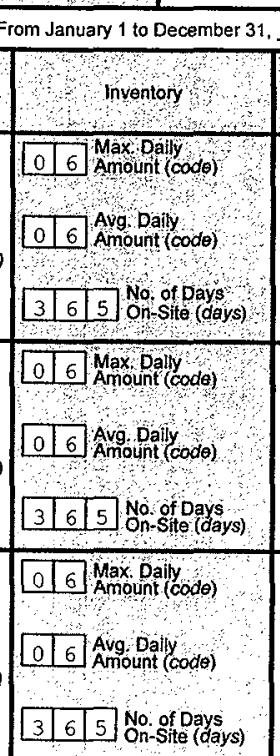
Cheak

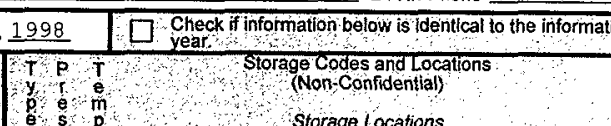
o $\mathrm{s}$

\begin{tabular}{|l|l|l|}
\hline$A$ & 1 & 4 \\
\hline
\end{tabular} NW CORNER OE 3020 . \begin{tabular}{lll|}
\hline $\mathrm{B}$ & 1 & 4 \\
\hline
\end{tabular} \begin{tabular}{|l|l|l|}
\hline$B$ & 1 & 4 \\
\hline
\end{tabular}

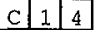
\begin{tabular}{l|l|l|} 
A & 1 & 4 \\
\hline
\end{tabular} \begin{tabular}{|l|l|l|}
\hline$D$ & 1 & 4 \\
\hline
\end{tabular}

\section{\begin{tabular}{|l|l|l|}
\hline$B$ & 1 & 4 \\
\hline
\end{tabular}} \begin{tabular}{l|l|l|}
\hline B & 1 & 4 \\
\hline
\end{tabular} \begin{tabular}{|l|l|l|}
\hline$B$ & 1 & 4 \\
\hline
\end{tabular} \begin{tabular}{|l|l|l|}
\hline $\mathrm{C}$ & 1 & 4 \\
\hline$B$ & & 4 \\
\hline
\end{tabular} \begin{tabular}{l|l|l|}
\hline $\mathrm{B}$ & 1 & 4 \\
\hline
\end{tabular} \begin{tabular}{|l|l|l|}
\hline$A$ & 1 & 4 \\
\hline
\end{tabular}

\begin{tabular}{|l|l|l|}
\hline$A$ & 1 & 4 \\
\hline$A$ & 1 & 4 \\
\hline$D$ & 1 & 4 \\
\hline & & \\
\hline & & \\
\hline & & \\
\hline
\end{tabular}
UNDERGROUND TANK N OF $3621 \quad 300$ AREA NDERGROUND TANK BY 3621D. 300 AREA $3621 D$ GENERATOR BUILDING/SHOP _ 300 AREA TANKS BY 382B PUMP STATION 300 AREA 384 STEAM PLANT, DEACTIVATED

UNDERGROUND TANK BY $408 \mathrm{~B}$ UNDERGROUND TANK BY. $408 B$ UNDERGROUND TANK BY $408 \mathrm{C}$ 427 BUIIDING 4721 GENERATOR BUIIDING 481 PUMP HOUSE BUIJDING $481 \mathrm{~A}$ PUMP HOUSE BUILDING 6618. DISPOSAL EACIIITY SE OE 200W AREA HTS PIPEYARD DRUM STORAGE NE OE 20OE AREA

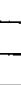
300 AREA 300 AREA $\square$ 400 AREA 400 AREA 400 ABEA 400 AREA 400 AREA 400 AREA $\square$ 400 AREA

EHS Name

Certification (Read and sign after completing all sections)

II certify under penalty of law that I have personally examined and am familiar with the information submitted in pages one through 70 inquiry of those individuals responsible for obtaining the information, I believe that the submitted information is true, accurate, and complete James E. Rasmussen, Director of Environmental Assurance, Permits and Policy Division IName and official title of owner/operator OR owner/operator's authorized representative

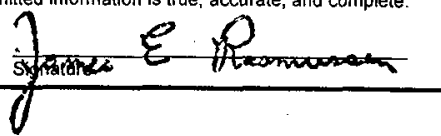

and that based on my

$02 / 26 / 99$ Date signed

\section{Optional Aftachments}

$\mathrm{X}$ I have attached a site plan I have attached a list of site coordinate abbreviations I have attached a description of dikes and other saleguard measures 


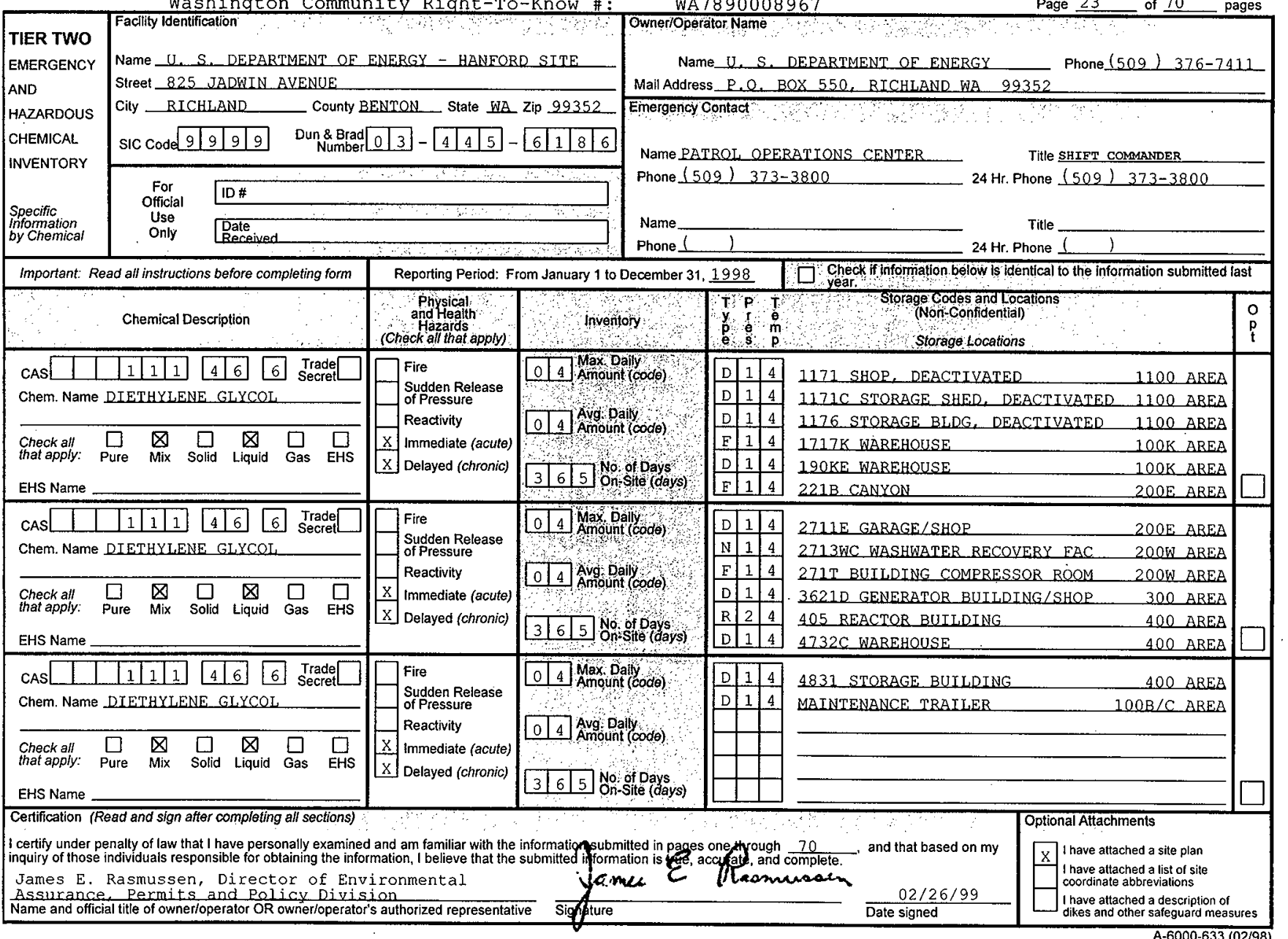


Washington Community Right-To-know \# :

WA7 890008967

Page 24 of 70 pages Owner/Operator Name

Name_U, S, DEPARTMENT OF ENERGY Phone $(509) \quad 376-7411$

Name $U$. S. DEPARTMENT OF ENERGY - HANEORD SITE Street 825 JADWIN AVENUE

City RICHLAND ____ County BENTON__ State WNA Zip 99352 Mail Address $\& .0$, BOX 550, RICHLAND WA 99352 Emergency Contact SIC Code \begin{tabular}{|l|l|l|l|}
9 & 9 & 9 & 9 \\
\hline
\end{tabular} \begin{tabular}{|l|l|l|l|l|l|l|} 
Dun \& Brad & 0 & 3 \\
Number & 0 & 3 & 4 & 5 \\
\hline
\end{tabular} CHEMICAL INVENTORY

$\begin{array}{ll}\begin{array}{c}\text { For } \\ \text { Official } \\ \text { Use } \\ \text { Only }\end{array} & \text { Date } \\ & \text { Daceived }\end{array}$

Name PATROL ORERATIONS CENTER Phone (509) 373-3800

Name

Phone ( )
Reporting Period: From January 1 to December 31, 1998 ahyslcal (Chock all that apply)

Chemicál Description

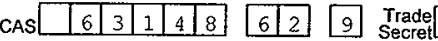

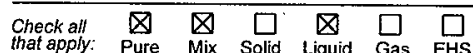
Chem. Name DIROTASSIUM RHOSPHATE Chem. Name DIMETHYI, SILOXANE EHS Name

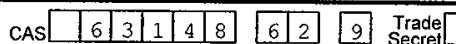
Chem. Name DIMETHYI, SILOXANE

\begin{tabular}{llllll}
\hline Check all & $\bigotimes$ & $\bigotimes$ & $\square$ & $\square$ & $\square$
\end{tabular} that apoly: Pure Mix Solid Liquid Gas EHS

EHS Name CAS \begin{tabular}{|l|l|l|l|l|l|l|}
\hline & 7 & 7 & 5 & 8 \\
\hline
\end{tabular}

$\square$ Fire Sudden Release
of Pressure Reactivity Immediate (acule) $\mathrm{X}$ Delayed (chronic)

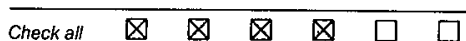
that apply: Pure Mix Solid Liquid Gas EHS

EHS Name

\section{Fire}

Sudden Release of Pressure

Reactivity Immediate (acute) $\mathrm{X}$ Delayed (chronic)

\section{Fire}

Sudden Release of Pressure Reactivity $\mathrm{X}$ Immediate (acute) Delayed (chronic)

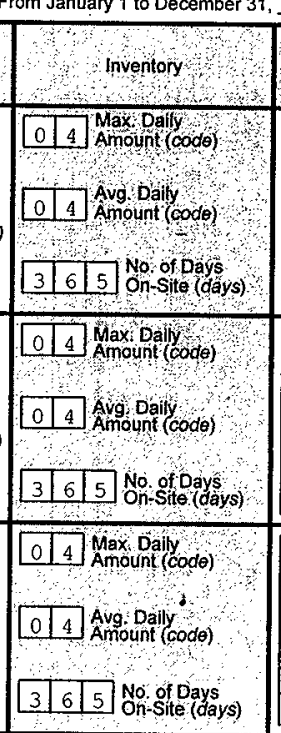

$\frac{1998}{T}$

y o

\begin{tabular}{|l|l|l|}
\hline$D$ & 1 & 4 \\
\hline$M$ & 1 & 4 \\
\hline$F$ & 1 & 4 \\
\hline$E$ & 1 & 4 \\
\hline$F$ & 1 & 4 \\
\hline$M$ & 1 & 4 \\
\hline
\end{tabular}

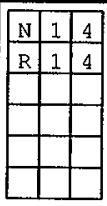

\begin{tabular}{|l|l|l|}
\hline$D$ & 1 & 4 \\
\hline$M$ & 1 & 4 \\
\hline$N$ & 1 & 4 \\
\hline$D$ & 1 & 4 \\
\hline$R$ & 1 & 4 \\
\hline$D$ & 1 & 4 \\
\hline
\end{tabular}

190KE WAREHOUSE 222SA LAB

$222 \mathrm{SA} L \mathrm{LAB}$

$2336 \mathrm{~W}$ SHOP

$2336 \mathrm{~W}$ BUILDING SYSTEM

STORAGE UNIT BY $241 \mathrm{~A} 401$

\section{Certification (Read and sign after completing afl sections)}

I certify under penalty of law that I have personally examined and am familiar with the information submitted in pages one through 70 inquiry of those individuals responsible for obtaining the information, I believe that the submitted information is true, accurate, and complete

James E. Rasmussen, Director of Environmental Assurance, Permits and Policy Division. Name and official title of owner/operator OR owner/operator's authorized representative

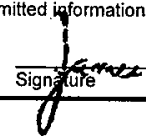

and that based on my
$02 / 26 / 99$

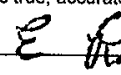

Title SHIFT COMMANDER 24 Hr. Phone (509) $373-3800$

Title
Check if information below is ifentical to the information submitted Storage Codes and Locations nfidential)

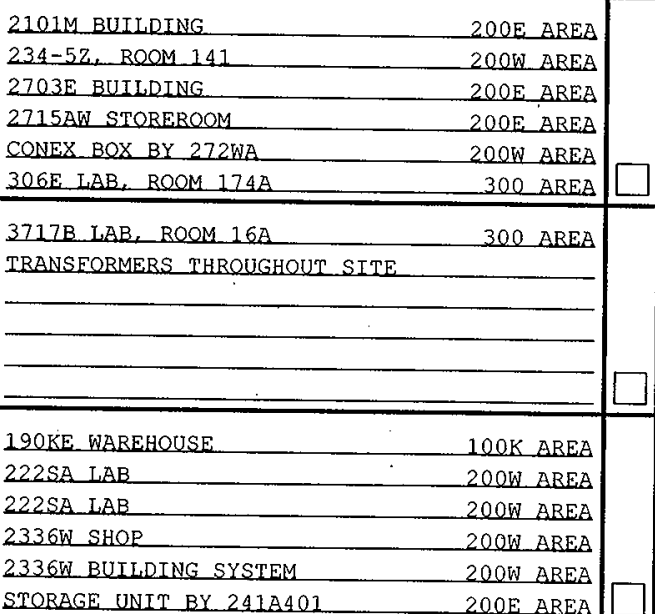
$200 \mathrm{E}$ AREA 
Washington Community Right-To-know \#:

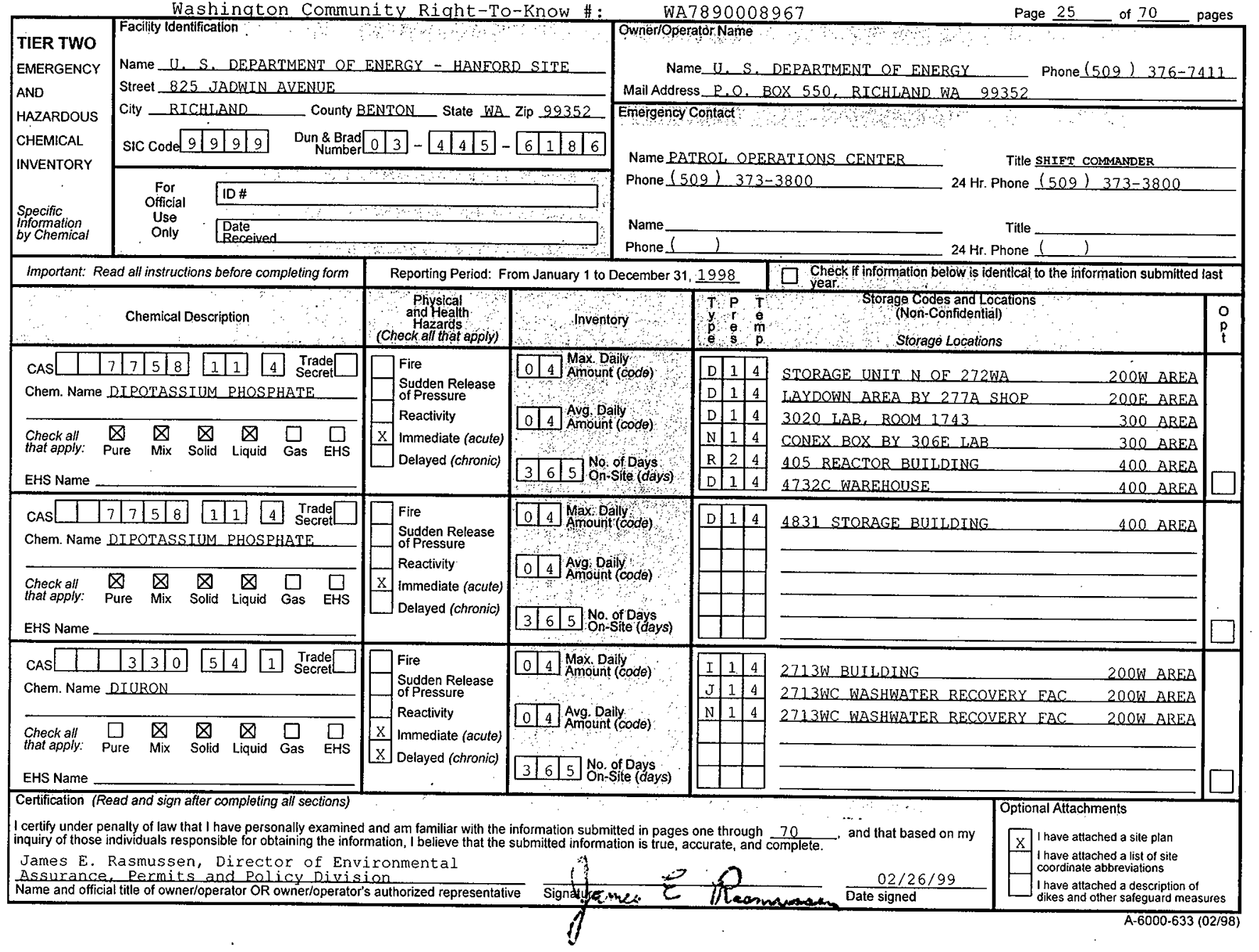




\section{TIER TWO}

EMERGENCY

AND

HAZARDOUS

CHEMICAL

INVENTORY

Name U.S. DEPARTMENT OF ENERGY - HANFORD SITE.

Street 825. JADWIN AVENUE

City _ RICHLAND C County BENTON

State WA Zip 29352

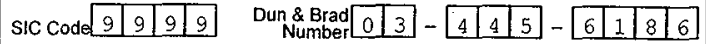

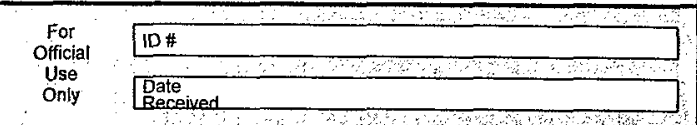
Specific
Information
by Chemica
Owner/Operator Name

Name_U.S. S. DEPARTMENT OE ENERGY Phone $(509) \quad 376-2411$ Mail Address_P.O. BOX 550, RICHLAND WA 99352 Emergency Contact

Name PATROL OPERATIONS CENTER

Title SHIFT COMMANDER

Phone (509) 373-3800 $24 \mathrm{Hr}$. Phone (509) $373-3800$

Name

Title

Phone L_ _ _ _ $24 \mathrm{Hr}$. Phone

Important: Read all instructions before completing form

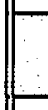

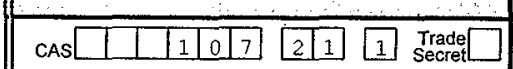
Chem. Name ETHYLENE GLYCOL

\begin{tabular}{lllllll}
\hline $\begin{array}{l}\text { Check all } \\
\text { that apply: }\end{array}$ & $\bigotimes$ & $\square$ & $\square$ & $\square$ & $\square$ & $\square$ \\
EHS Name & & & & & $\square$
\end{tabular}

Reporting Period: Fr
Physical
and Health
Hazards
(Check all that apply)
$\square$ Fire

Sudden Release
of Pressure
Reactivity

\begin{tabular}{|l|l|} 
& Reactivity \\
\hdashline$X$ & Immediate (acute) \\
\hline$X$ &
\end{tabular}

$\mathrm{X}$ Delayed (chronic)

\begin{tabular}{|l|l|l|l|l|l|l|l|l|l|}
\hline CAS & & & & 1 & 0 & 7 \\
\hline
\end{tabular}

Chem. Name ETHYLENE GLYCOL

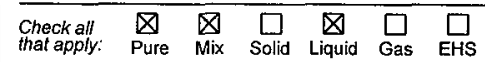

EHS Name

\begin{tabular}{|l|l|l|l|l|l|l|l|l|}
\hline CAS & & & & 1 & 0 & 7 \\
\hline
\end{tabular} Chem. Name ETHYLENE GLYCOI,

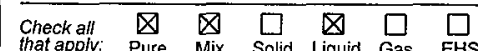

That apply: Pure Mix Solid Liquid Gas EHS

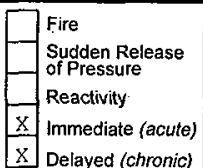

Fire

\begin{tabular}{|l|l|}
\hline & $\begin{array}{l}\text { Sudden Release } \\
\text { of Pressure } \\
\text { Reactivity }\end{array}$ \\
\hline $\mathrm{X}$ & $\begin{array}{l}\text { Immediate (acute) } \\
\text { Im }\end{array}$ \\
Delayed (chronic)
\end{tabular}

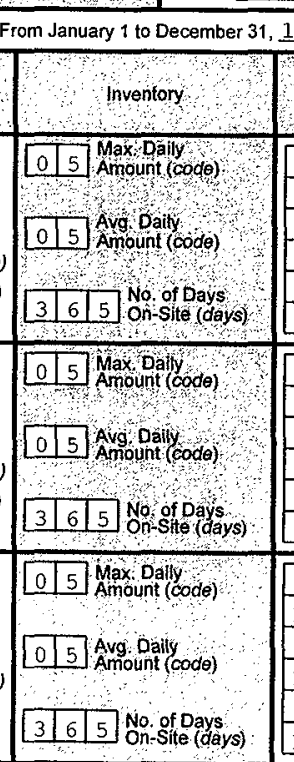

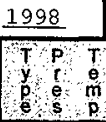

7 Chear. if information below is ldentical to the information submitted last Certification (Read and sign after completing all sections)

Storagecodes and Locations
(Non-Confidential)

Storage Locations.

\begin{tabular}{|l|l|l|l|l|l|l|}
\hline $\mathrm{N}$ & 1 & 4 & $1143 \mathrm{~N}$ SHOP & $100 \mathrm{~N}$ AREA
\end{tabular}

\begin{tabular}{ll|l|ll}
$\mathrm{N}$ & 1 & 4 & $1143 \mathrm{~N}$ SHOP & $100 \mathrm{~N}$ AREA \\
\cline { 1 - 1 } & 1 & 4 & &
\end{tabular}

1100 AREA

D I $1171 \mathrm{C}$ STORAGE SHED, DEACTIVATED 1100 AREA

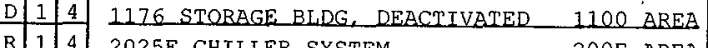

\begin{tabular}{ll|lll}
$\mathrm{R}$ & 1 & 4. & $2025 \mathrm{E}$ CHILLER SYSTEM _ 200E AREA
\end{tabular}

$2101 \mathrm{HV}$ WAREHOUSE $200 \mathrm{E}$ AREA

\begin{tabular}{|l|l|l|l|}
\hline$D$ & 1 & 4 \\
\hline$M$ & 1 & 4 & $2101 \mathrm{M}$ WAREHOUSE \\
\hline
\end{tabular}

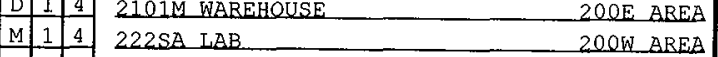

$D$ 1 4 CONEX BOX S OF 234-5Z 200W AREA

M 114 234-57, ROOM 183_200W_AREA

\begin{tabular}{lllll}
$\mathrm{N}$ & 1 & 4 \\
\hline
\end{tabular}

\begin{tabular}{|l|l|l|l|l}
\hline$D$ & 1 & 4 & $2711 \mathrm{E}$ GARAGE/SHOP & $200 \mathrm{E}$ AREA \\
\hline
\end{tabular}

\begin{tabular}{|l|l|l|l|llll}
\hline $\mathrm{N}$ & 1 & 4 & 2713WC WASHWATER RECOVERY EAC & 200W AREA \\
\hline $\mathrm{N}$ & 1 & 4 & $2715 \mathrm{~W}$ & STOREROQM
\end{tabular}

\begin{tabular}{ll|l}
$\mathrm{N}$ & 1 & 4 \\
\hline
\end{tabular}

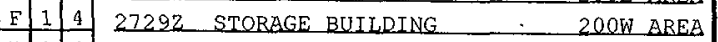

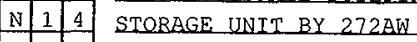
$200 E$ AREA

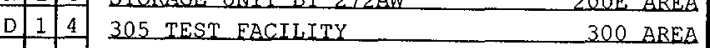

\begin{tabular}{llll}
\hline E & 1 & 4 & \\
\hline
\end{tabular}

I certify under penalty of law that I have personally examined and am familiar with the information submitted in pages one through 70

inquiry of those individuals responsible for obtaining the information, I believe that the submitted information is truge, accurate, and complete

James E. Rasmussen, Director of Environmental

Assurance, Permits and Policy Division

Name and official title of owner/operator OR owner/operator's authorized representative

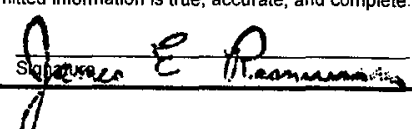

$02 / 26 / 99$ 


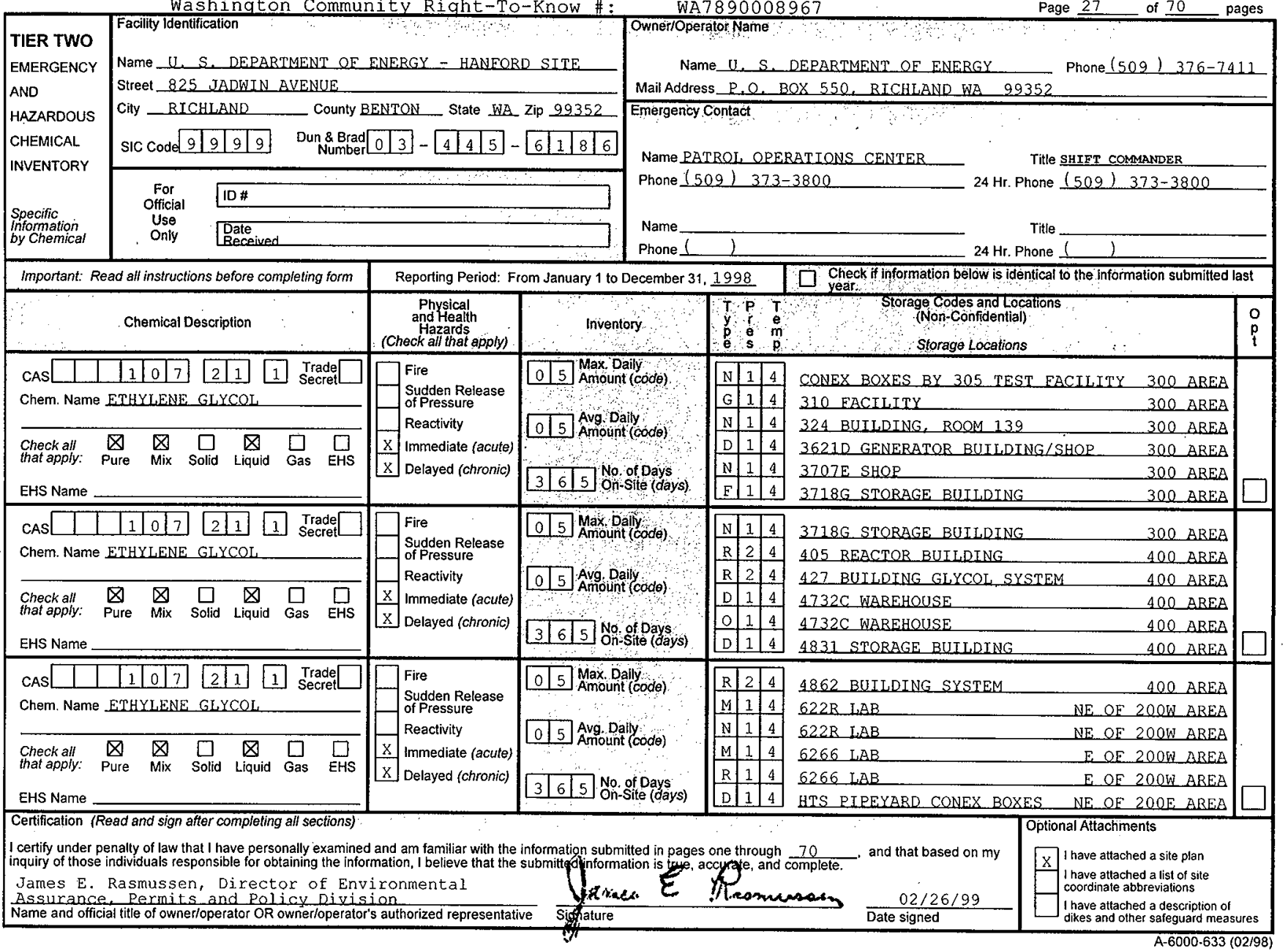


Washington Community Right-To-know \#:

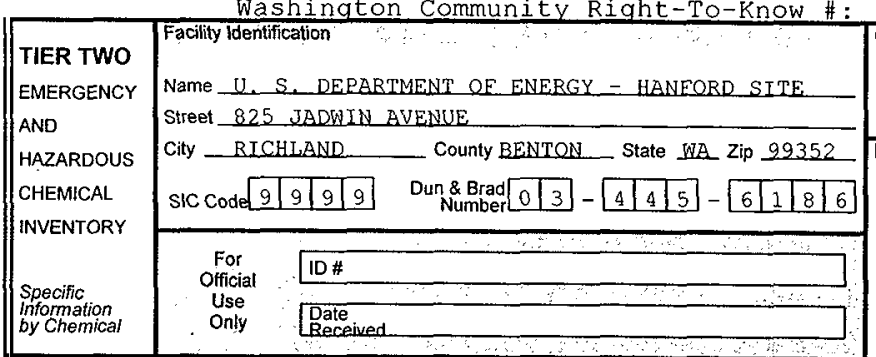

WA7 890008967 Owner/Operator Name

Name_U._S. DERARTMENT OF ENERGY Mail Address_P.O, BOX 550, RICHLAND WA 99352 Emergency Contact:

Name PATROL ORERATIONS CENTEB

Phone_(509) $373-3800$

Title SHIFT COMMANDER $24 \mathrm{Hr}$. Phone (509) 373-3800

Name Phone $(1$ Title $24 \mathrm{Hr}$. Phone 1

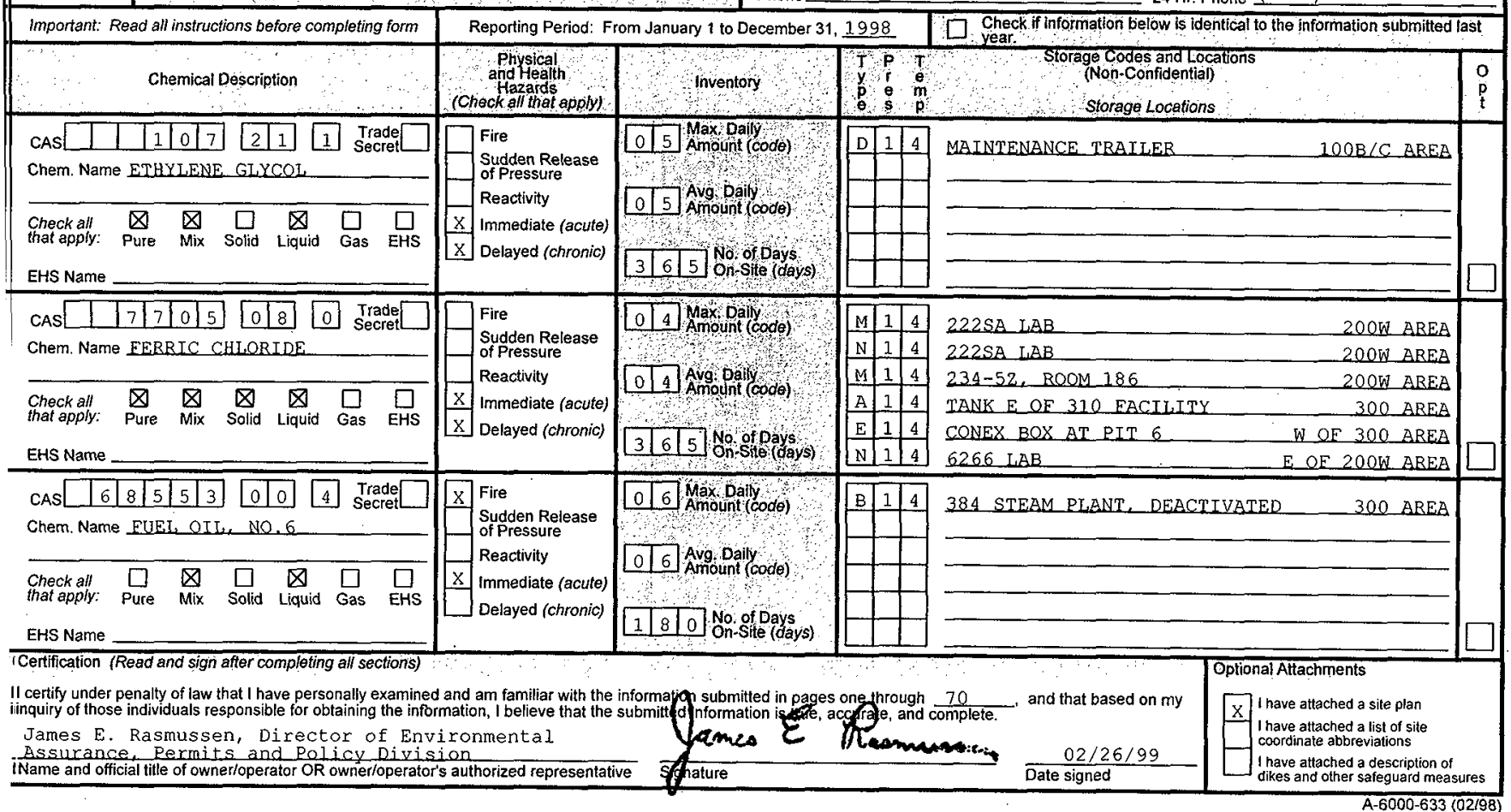




\section{TIER TWO}

EMERGENCY

AND

HAZARDOUS

CHEMICAL

INVENTORY

Specific
information
by Chemical

Name $U$. S. DEPARTMENT OF ENERGY - HANEORD SITE

Street 825 JADWIN AVENUE

City RICHIAND County BENTON_ State WA Zip 99352

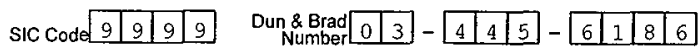

For
Official
Use
Only

\section{ID \#}

Date

\section{Owner/Operator Name}

Name_U. $S$. DEPARTMENT OE ENERGY Mail Address P.O. BOX 550, RICHLAND WA 99352

Phone (50.9) $376-7411$ Emergency Contact
Name PATROL OPERATIONS. CENTER

Phone (509) 373-3800

Name

Phone ( )
Title SHIFT COMMNDER $24 \mathrm{Hr}$. Phone $(509) \quad 373-3800$

Title $24 \mathrm{Hr}$. Phone
Important: Read all instructions before completing form Chemical Description

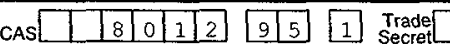
Chem. Name MINERAL OIL

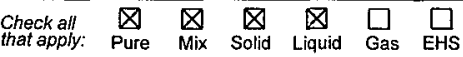

EHS Name

CAst \begin{tabular}{ll|l|l|l|l|l|l|l|}
\hline & 8 & 0 & 1 & 2 \\
\hline
\end{tabular} Chem. Name MINERAL QIL

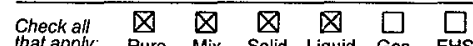
that apply: Pure Mix Solid Liquid Gas EHS EHS Name \begin{tabular}{l|l|l|l|l|l|l|l|l|}
\hline CAS & & 8 & 0 & 1 & 2 \\
\hline
\end{tabular}

Chem. Name MINERAI OLL.

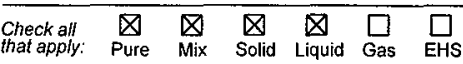

EHS Name

Certification (Read and sign after complating all sections)

Physical and Healt anarads (Chock all that apply)}

Reporting Period: From January 1 to December 31, 1998

\section{Fire}

Sudden Release of Pressure

Reactivity

$\mathrm{X}$ Immediate (acute)

$x$ Delayed (chronic)

\section{Fire}

Sudden Release of Pressure

Reactivity $x$ Immediate (acute) $x$ Delayed (chronic)

\section{Fire}

Fire
Sudden Release

of Pressure

Reactivity

$\mathrm{X}$ Immediate (acute)

$x$ Delayed (chronic)

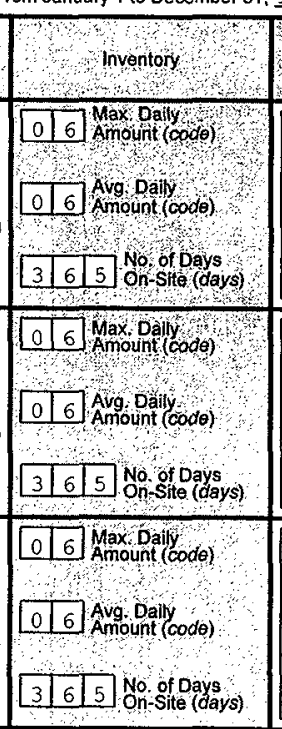

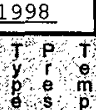

\begin{tabular}{|l|l|l|}
\hline 0 & 1 & 4 \\
\hline
\end{tabular}

\begin{tabular}{|l|l|l|}
\hline$D$ & 1 & 4 \\
\hline & 1 & 4 \\
\hline
\end{tabular} \begin{tabular}{|l|l|l|}
\hline $\mathrm{N}$ & 1 & 4 \\
\hline $\mathrm{D}$ & 1 & 4 \\
\hline
\end{tabular} \begin{tabular}{|l|l|l|}
\hline$D$ & 1 & 4 \\
\hline
\end{tabular}

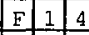
\begin{tabular}{|l|l|l|}
\hline $\mathrm{M}$ & 1 & 4 \\
\hline
\end{tabular}

\begin{tabular}{|l|l|l|}
\hline$E$ & 1 & 4 \\
\hline$E$ & 1 & 4 \\
\hline$M$ & 1 & 4 \\
\hline$M$ & 1 & 4 \\
\hline$R$ & 1 & 4 \\
\hline
\end{tabular}

\begin{tabular}{|l|l|l|}
\hline$D$ & 1 & 4 \\
\hline
\end{tabular}

\begin{tabular}{|c|c|c|}
\hline$M$ & 1 & 4 \\
\hline$G$ & 1 & 4 \\
\hline$M$ & 1 & 4 \\
\hline$M$ & 1 & 4 \\
\hline E & 1 & 4 \\
\hline$D$ & 1 & 4 \\
\hline
\end{tabular}

100-DR-1 OPERABLE UNIT $105 \mathrm{NB}$ SHOP 1171 SHOP, DEACTIVATED $1171 \mathrm{C}$ STORAGE SHED, DEACTIVATED 1100 AREA STORAGE UNIT SW OE $1724 \mathrm{~K}$ STORAGE UNIT SW OE $1724 \mathrm{~K}$ $100 K$ AREA 100K AREA

$183 \mathrm{KE}$ WATER PLANT LOOK_AREA $190 \mathrm{KE}$ WAREHOUSE 10OK AREA I $90 \mathrm{KE}$ WAREHOUSE STORAGE,UNIT BY 201W 100K AREA $200 W$ AREA YARD W OF 2101M 200E AREA STORAGE UNIT SW OE 211T 200W AREA $214 \mathrm{~T}$ STORAGE BUILDING 200W AREB $221 \mathrm{~T}$ CANYON 200W AREA $222 \mathrm{~S} \quad \mathrm{LAB}$ $234-52$, CORRIDOR 4 200W AREA 200W AREA $2706 \mathrm{~T}$ BUILDING $200 W$ AREA 2711 E GARAGE/SHOP $200 E$ ABEA

II certify under penalty of law that I have personally examined and am familiar with the information submitted in pages one through 70 inquiry of those individuals responsible for obtaining the information, I believe
James E. Rasmussen; Director of Envi ronmental Assurance, Permits and Policy Division Name and official title of owner/operator OR owner/operator's authorized representative

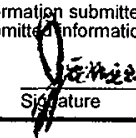
and that based on my Date signed

Optiona
attachments
I have attached a site plan I have attached a list of site coordinate abbreviations dikes and other safeguard measure thave attached a description of 
Washington Community Right-To-Know \#:

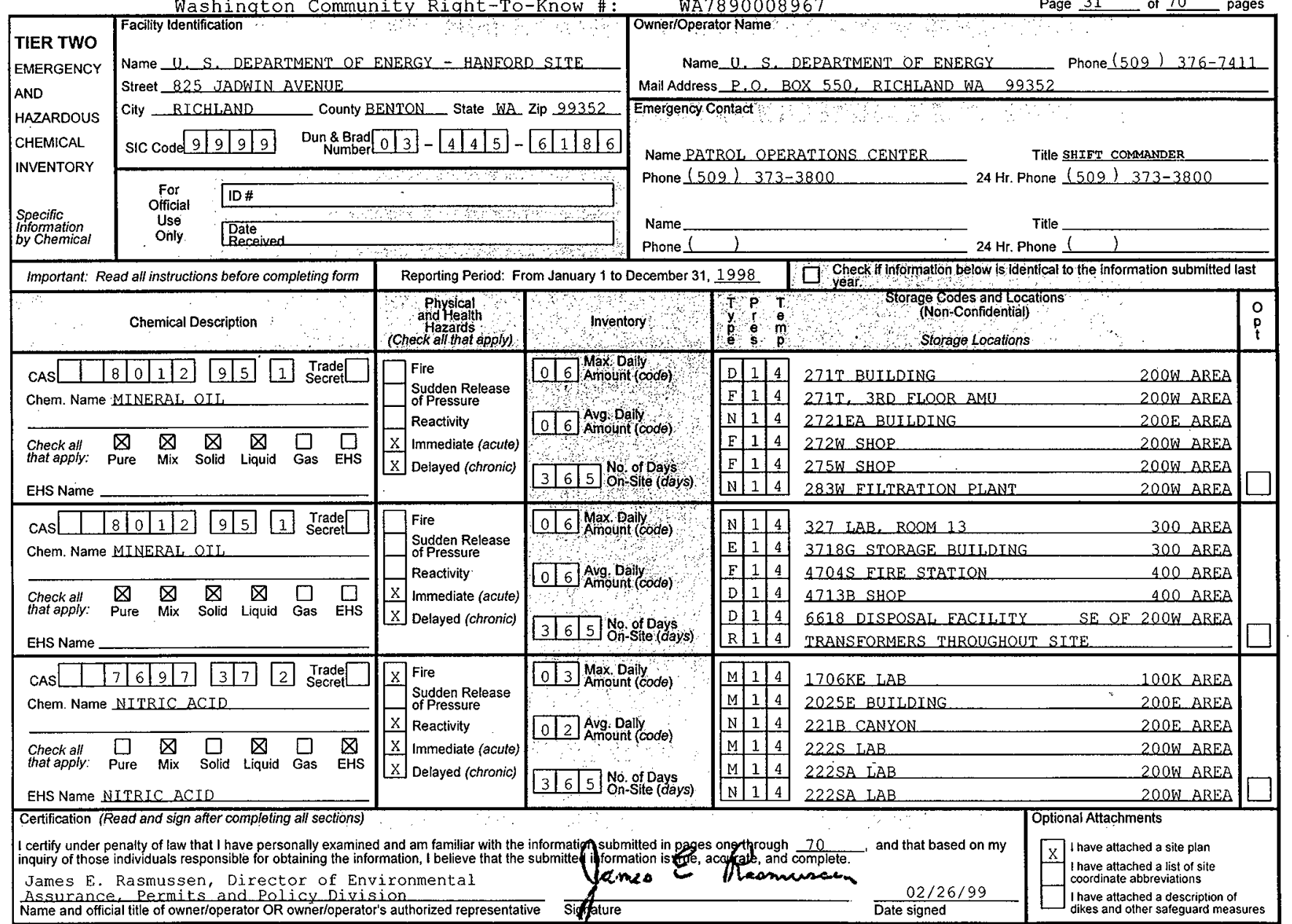


Washington Community Right-To-Know \#:

\section{TIER TWO}

EMERGENCY

AND

HAZARDOUS

CHEMICAL

Specific
information
by Chemical
INVENTORY
Name U. $S$ DEPARTMENT OF ENERGY - HANFORD SITE

Street 825 JADWIN_AVENUE

City BICHLAND _ County BENTON State WA Zip 29352

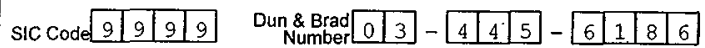

WA7 890008967

Owher/Operator Name

Name_U. S. DEPARTMENT OF ENERGY Mail Address _. . O . BOX 550, RICHLAND WA 99352 Emergency Contact

Name PATROL ORERATIONS CENTER

Phone (509) 373-3800

Title SHIET COMMANDER $24 \mathrm{Hr}$. Phone $(509) \quad 373-3800$

Name

Title

Phone 1 ( 24 Hr. Phone $(1$

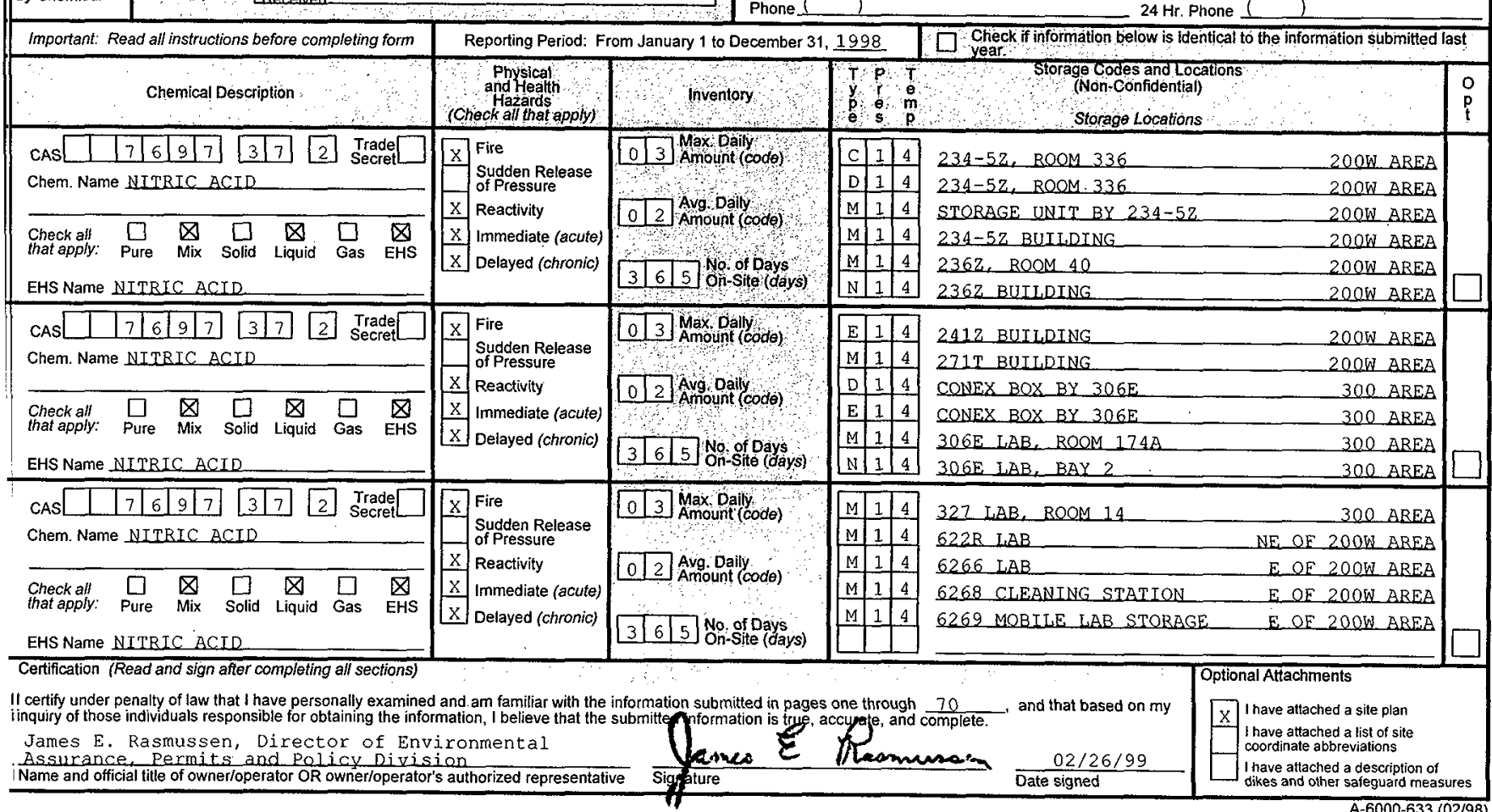




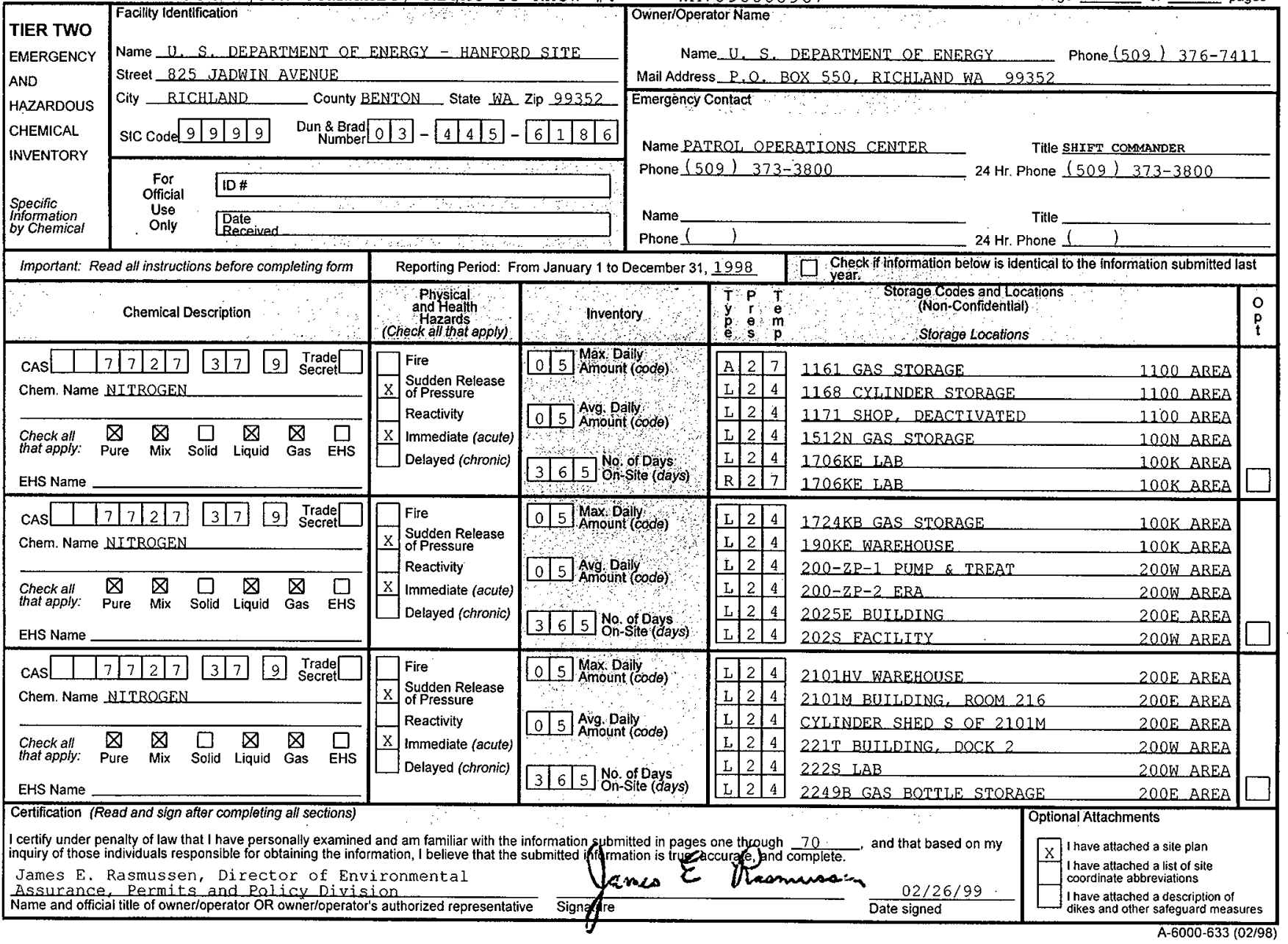


Washington Community Right-To-know \#:

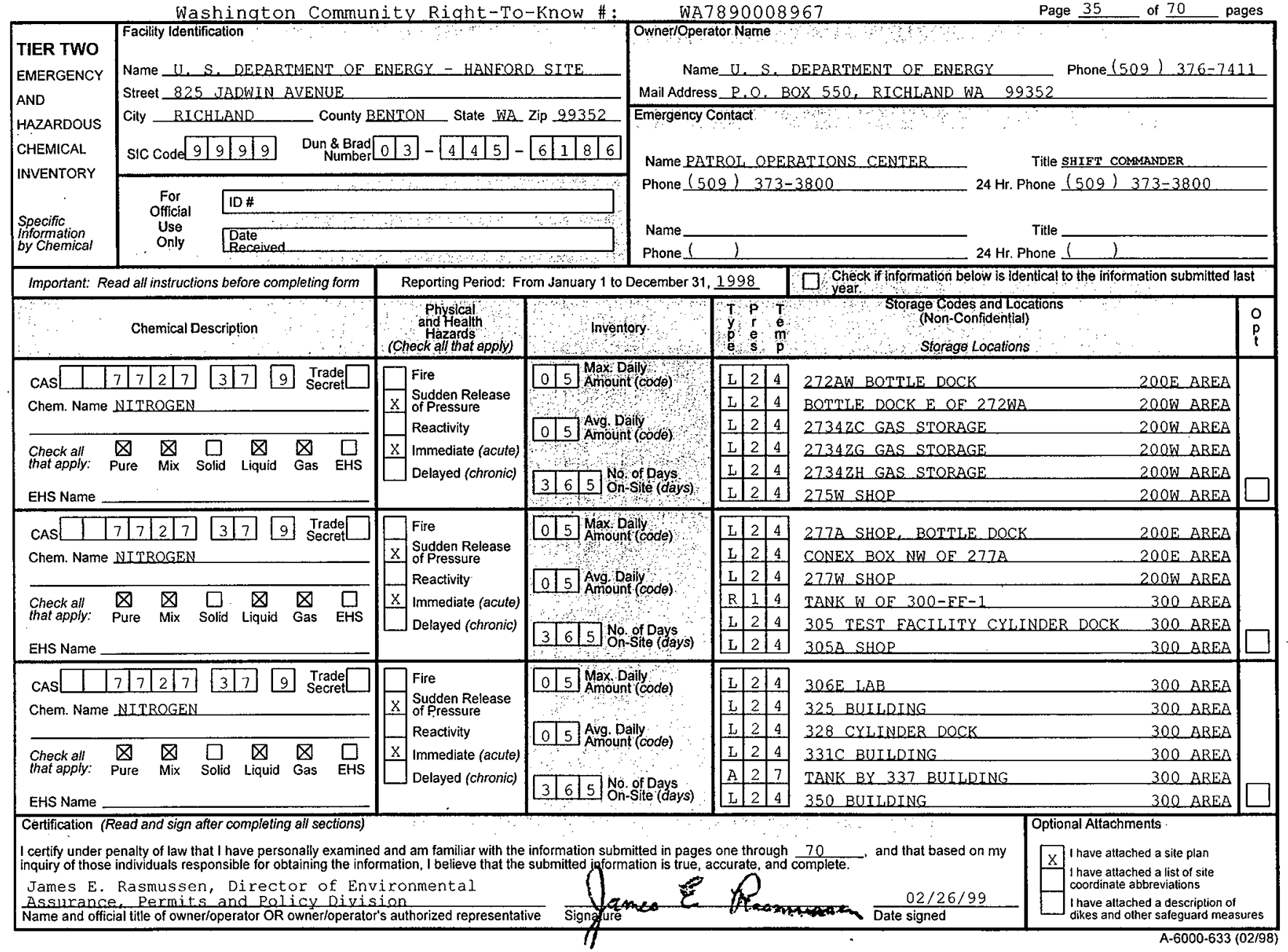




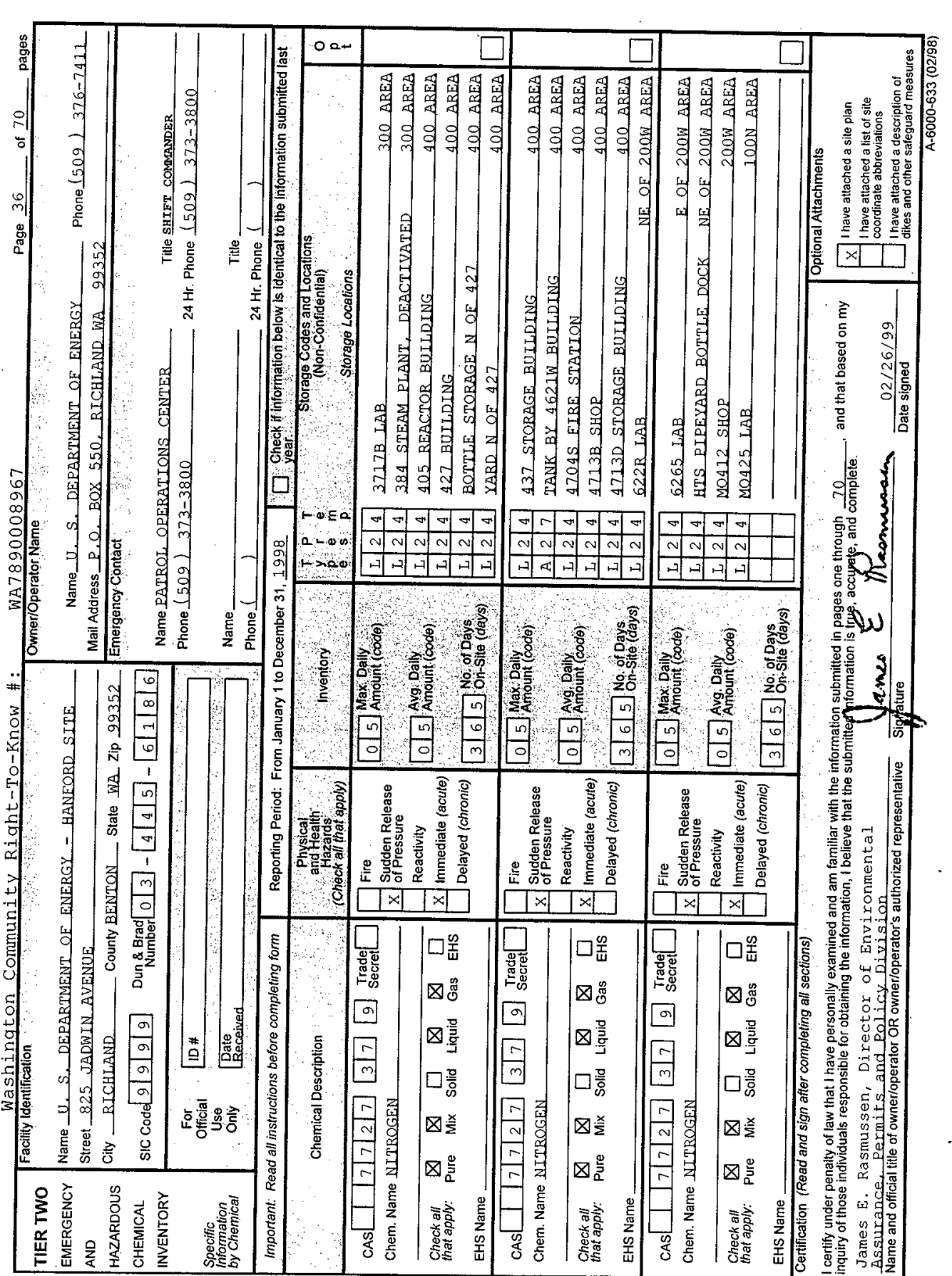


Washington Community Right-To-know \#:

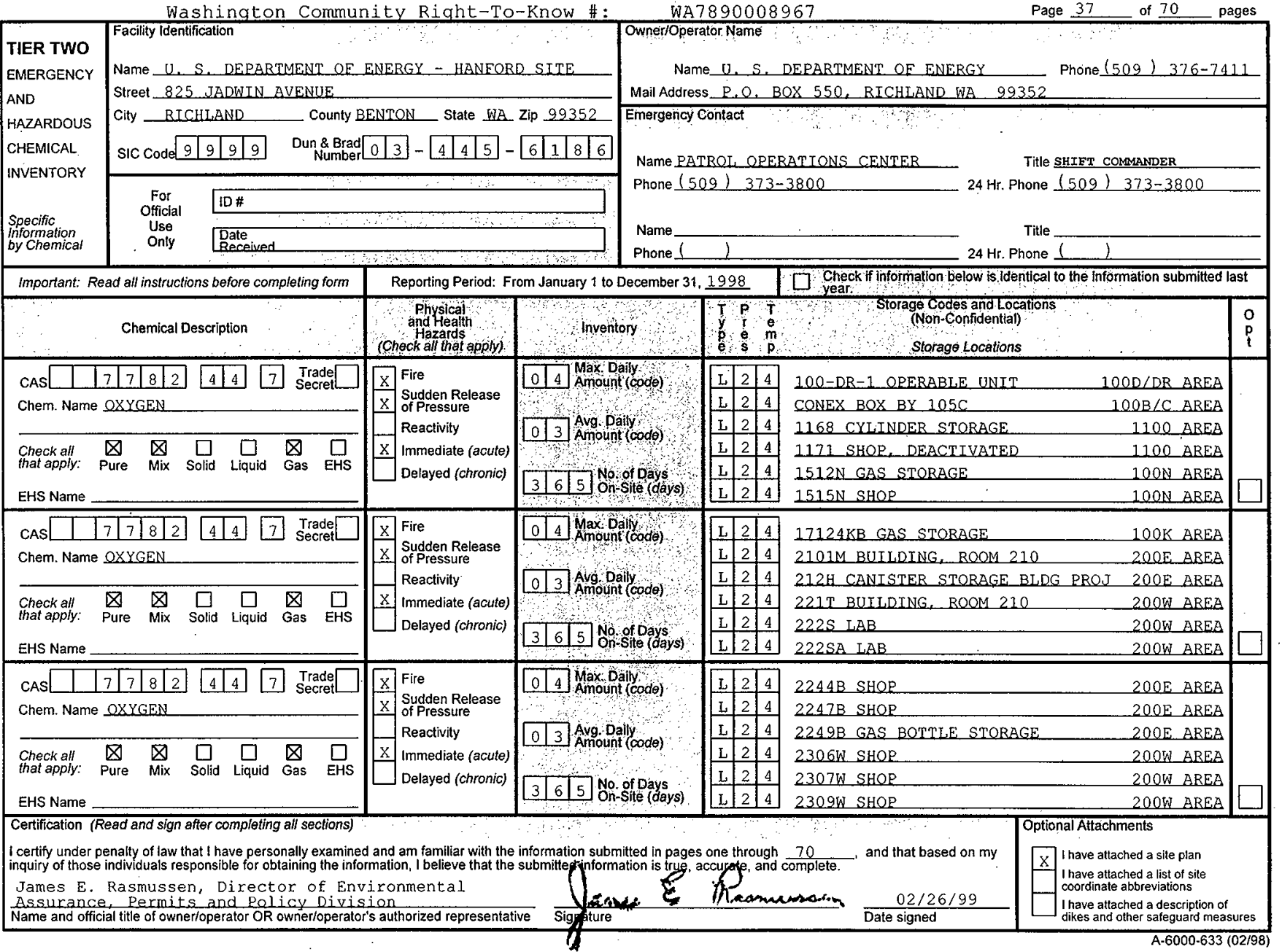




\section{TIER TWO}

EMERGENCY

AND

HAZARDOUS

CHEMICAL

INVENTORY

Specific

Information
Name U. S. DEPARTMENT OE ENERGY - HANFORD SITE Street 825 JADWIN AVENUE,

City RICHLAND ___ County BENTON State WA Zip 99352

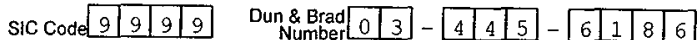

For
Official
Use
Only
Only

Owner/Operator Name

Name_U. S. DEEARTMENT OF ENERGY Phone (509) $376-7411$ Mail Address_P.O BOX 550, RICHLAND WA 99352 Emergency Contact

Name PATROL OPERATIONS CENTER Phone (509) 373-3800

Name Phone (
Title SHIFT COMMANDER $24 \mathrm{Hr}$. Phone $(509) 373-3800$

Titte $24 \mathrm{Hr}$. Phone

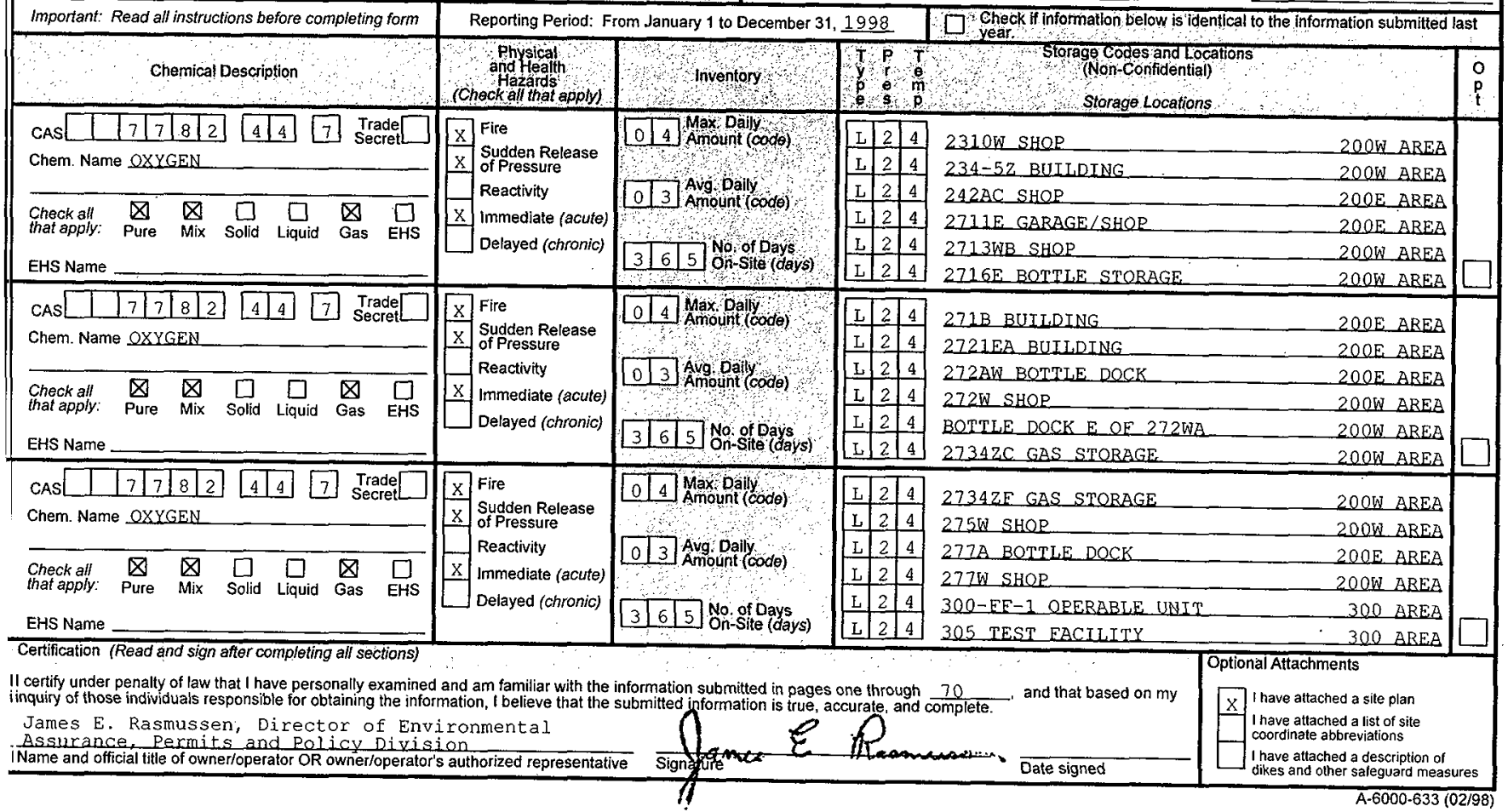




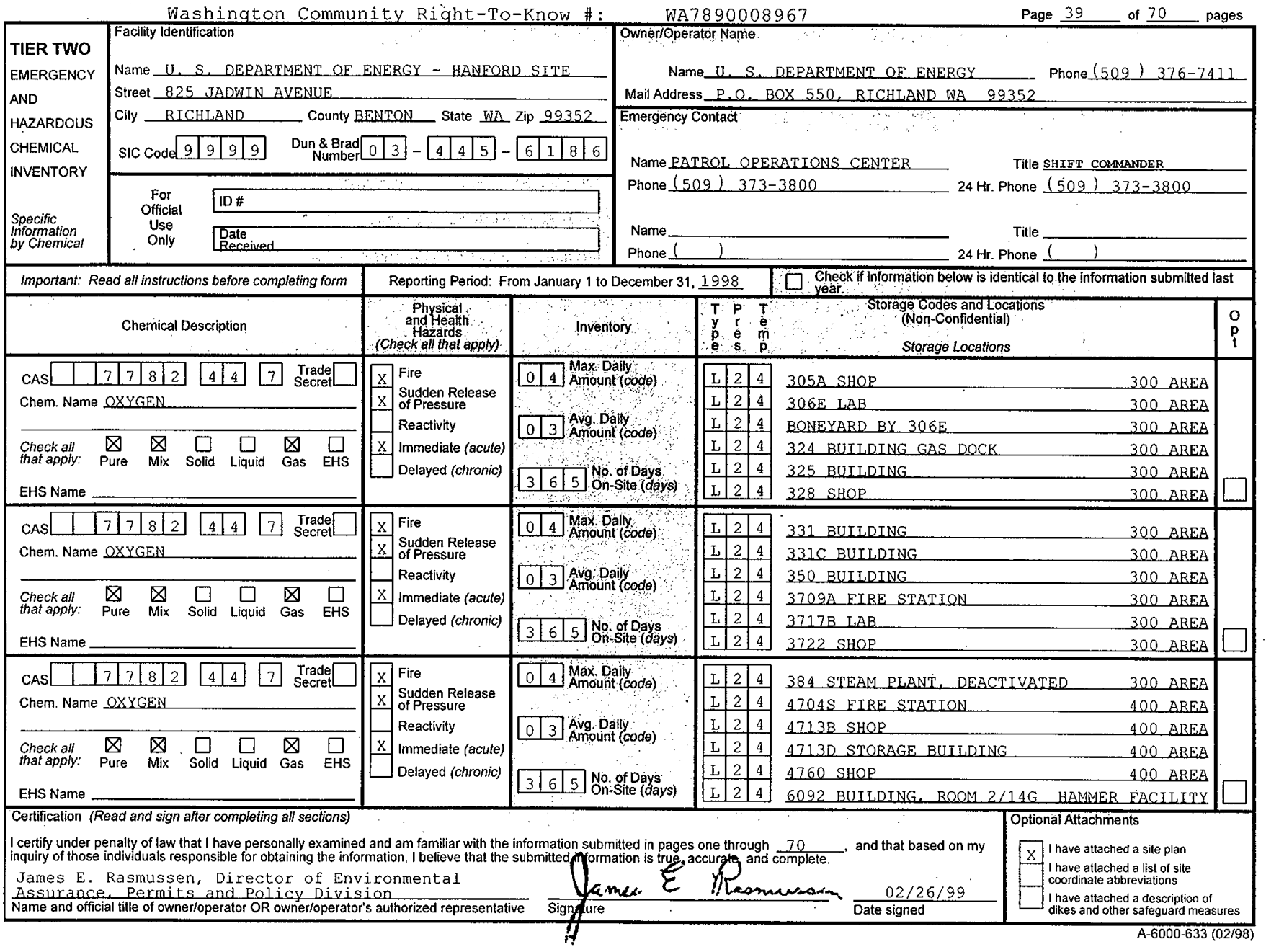




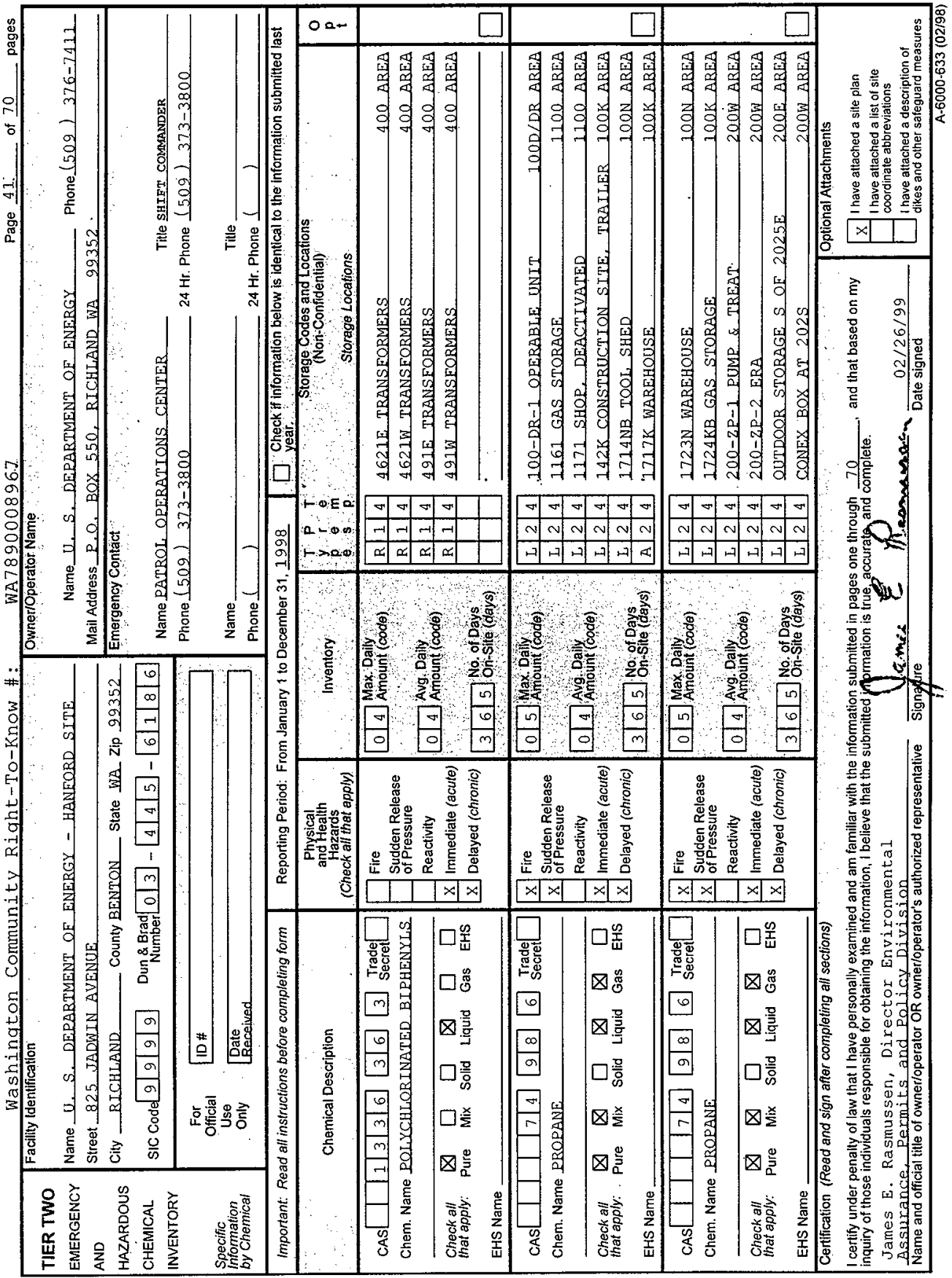




\section{TIER TWO}

EMERGENCY

AND

HAZARDOUS

CHEMICAL

Specific

Information

by Chemical
INVENTORY
Name U.S. DE. DERTMENT OF ENERGY - HANFORD SITE

Street 825 JADN IN AVENUE

City _RICHLAND _ C County BENTON State WA Zip 99352

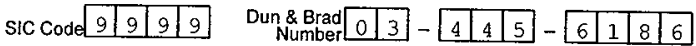

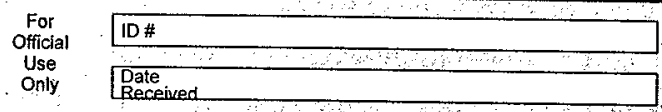

Name_U. S. DERARTMENT OF ENERGY Phone (509) $376-7411$ Mail Address P.O. BOX 550, RICHLAND WA 99352 Emergency Contact
Name PATROL ORERATIONS CENTER

Phone $(.509) \quad 373-3800$

Name

Phone
Title SHIFT COMMANDER $24 \mathrm{Hr}$. Phone (509) 373-3800 $24 \mathrm{Hr}$. Phone $(1)$
Important: Read all instructions before completing form

Chemical Description

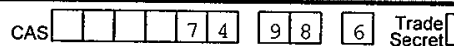
Chem. Name PROPANE

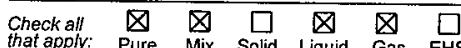
EHS Name CAS \begin{tabular}{l|l|l|l|l|l|l|l|l|l|l|}
\hline & & & & 7 & 4 & 9 & 8 \\
\hline
\end{tabular}

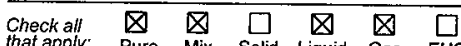
that apply: Pure Mix Solid Liquid Gas EHS

EHS Name

CAS \begin{tabular}{l|l|l|l|l|l|l|l|l|}
\hline & & & & 7 & 4 \\
\hline 9 & 8 & 6 \\
\hline
\end{tabular} $\begin{aligned} & \text { Trade } \\
& \text { Secret }\end{aligned}$ Chem. Name PROPANE

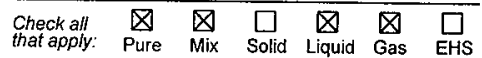

EHS Name (Certification (Read and sign after completing all sections). Chem. Name PROPANE
Reporting Period: From January 1 to December 31, 1998

Physical and Health (Check all that apply)

$x$ Fire

$x$ Sudden Release

$X$ of Pressure

Reactivity

$\mathrm{X}$ immediate (acute)

$\mathrm{X}$ Delayed (chronic)

\section{$\mathrm{x}$ Fire}

$X$ Sudden Release

$X$ of Pressure

Reactivity

$\mathrm{X}$ Immediate (acute)

$\mathrm{X}$ Delayed (chronic)

\begin{tabular}{|c|c|}
\hline 811 & Fire \\
\hline$x$ & $\begin{array}{l}\text { Sudden Release } \\
\text { of Pressure }\end{array}$ \\
\hline & Reactivity \\
\hline & ediate \\
\hline & \\
\hline
\end{tabular}

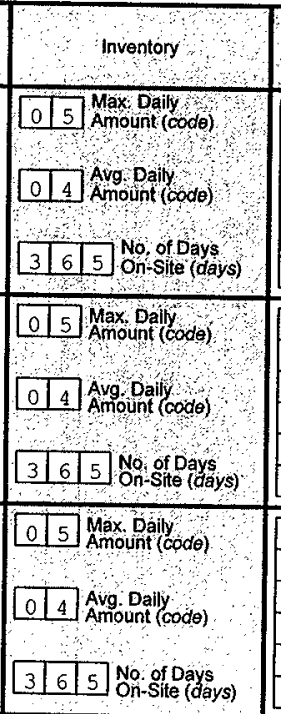

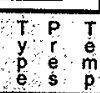

\begin{tabular}{|l|l|l|}
\hline $\mathrm{L}$ & 2 & 4 \\
\hline & 2 & 4 \\
\hline
\end{tabular}

\begin{tabular}{l|l|l|}
\hline$L$ & 2 & 4 \\
\hline & & 2 \\
\hline
\end{tabular}

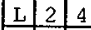

\begin{tabular}{l|l|l|} 
A & 2 & 4 \\
\hline & & 2 \\
\hline
\end{tabular}

\begin{tabular}{l|l|l|}
\hline $\mathrm{L}$ & 2 & 4 \\
\hline & & 2 \\
\hline
\end{tabular}

\begin{tabular}{|l|l|l|}
\hline $\mathrm{L}$ & 2 & 4 \\
\hline
\end{tabular}

\begin{tabular}{|l|l|l|}
\hline$L$ & 2 & 4 \\
\hline$L$ & 2 & 4 \\
\hline$L$ & 2 & 4 \\
\hline$L$ & 2 & 4 \\
\hline$L$ & 2 & 4 \\
\hline$A$ & 2 & 4 \\
\hline
\end{tabular}

\begin{tabular}{l|l|l|}
\hline$A$ & 2 & 4 \\
\hline
\end{tabular}

\begin{tabular}{|l|l|l|}
\hline $\mathrm{L}$ & 2 & 4 \\
\hline
\end{tabular} \begin{tabular}{llll}
$\mathrm{L}$ & 2 & 4 & $2306 \mathrm{~W}$ SHOP \\
\hline
\end{tabular}

\begin{tabular}{lll|}
\hline $\mathrm{L}$ & 2 & 4 \\
\hline
\end{tabular}

\begin{tabular}{lll|l}
\hline $\mathrm{L}$ & 2 & 4 \\
\hline
\end{tabular}

\begin{tabular}{l|l|l|}
\hline & 2 & 4 \\
\hline
\end{tabular}
Cerigh atier completing all sections)$$
1
$$

:

\section{$\square$ chear}

information below is identical to the information submitted last
Storage Codes and Locations
\begin{tabular}{l|l} 
(Non-Confidential) & 0 \\
Storage Locations & $t$ \\
\hline
\end{tabular}

$200 E$ AREA 200E AREA $200 E$ AREA $212 \mathrm{H}$ CANISTER STORAGE BLDG PROJ $200 \mathrm{E}$ AREA $212 \mathrm{H}$ CANISTER STORAGE BLDG PROJ 200E AREA 221T BUILDING, DOCK 2 200W AREA $\square$

222S: LAB, GAS DOCKS 200W AREA $200 E$ AREA 200 E AREA $200 E$ AREA $200 E$ AREA 200E AREA 20OW AREA 200W AREA 200W AREA 200W AREA 200W AREA 200W AREA
II certify under penalty of law that I have personally examined and am familiar with the information submitted in pages one through 70 James E. Rasmussen, Director Environmental
Assurance, Permits and Policy Division iName and official title of ownerfoperator OR owner/operator's authorized representative

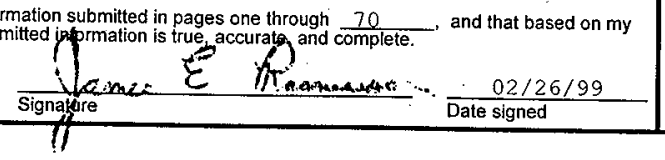


Washington Community Right-To-know \#:

\section{TIER TWO}

EMERGENCY

AND

HAZARDOUS

CHEMICAL

INVENTORY

Specific

information

by Chemical Faclity ldentification

Name $U$. $S$. DEPARTMENT OF ENERGY - HANFORD SITE

Street 825 JADWIN AVENUE

City RICHLAND.

County BENTON - State WA Zip 99352

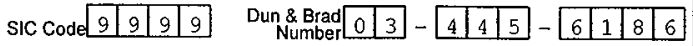

\begin{tabular}{lll} 
For & ID $\#$ \\
Official & \\
Use & \\
Oniy & Date & \\
\hline Received & \\
\end{tabular}

WA7890008967 Owher/operator Name

Name $U$. S. DEPARTMENT OF ENERGYY Page 43 of 70 pages Mail Address P.O. BOX 550, RICHLAND WA 99352

Phone (502) $376-7411$ Emergency Contact

Name RATROL OPERATIONS CENTER Phone (509) 373-3800

Name

Phone $(1)$
Title SHIFT COMMANDER $24 \mathrm{Hr}$. Phone $(509) \quad 373-3800$

Title $24 \mathrm{Hr}$. Phone (1)

Important: Read all instructions before completing form $\quad$ Reporting Period: From January 1 to December 31, 1998

\begin{tabular}{|cc|}
\hline & Chemical Description \\
\hline & \\
\hline
\end{tabular}

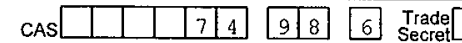

Chem. Name PROPANE

$\begin{array}{lllllll}\text { Check all } & \bigotimes & \bigotimes & \square & \bigotimes & \bigotimes & \square\end{array}$

that apply: Pure Mix Solid Liquid Gas EHS

EHS Name

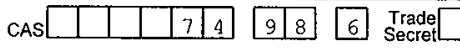
Chem. Name PROPANE

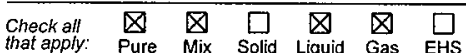

EHS Name

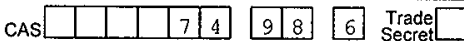

Chem. Name RBOPANE

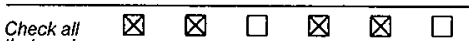

that apply: Pure Mix Solid Liquid Gas EHS

EHS Name

Certification (Read and sign after completing all sections)

Physical and Health (Check all that apply)

\section{$x$ Fire}

$\mathrm{X}$ Sudden Release

$x$ of Pressure

Reactivity

$\mathrm{x}$ Immediate (acute)

$x$ Delayed (chronic)

\begin{tabular}{|l|l|l|}
\hline $\mathrm{x}$ & $\begin{array}{l}\text { Fire } \\
\mathrm{x}\end{array}$ & $\begin{array}{l}\text { Sudden Release } \\
\text { of Pressure }\end{array}$ \\
$\mathrm{y}$ & $\begin{array}{l}\text { Reactivity } \\
\mathrm{X}\end{array}$ & Immediate (acute) \\
$\mathrm{x}$ & Delayed (chronic) \\
\hline
\end{tabular}

$x$ Fire

$x$ Sudden Releasie

$x$ of Pressure

Reactivity

$\mathrm{X}$ Immediate (acute)

$\mathrm{X}$ Delayed (chronic)

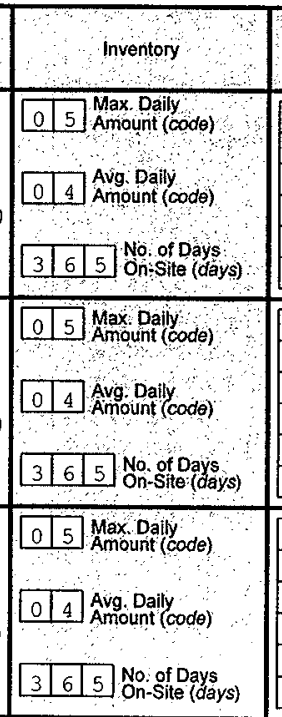

$T P$

$\begin{array}{lll}y & o \\ \text { p } & \mathrm{m} \\ \mathrm{p} & \mathrm{p}\end{array}$

\begin{tabular}{|l|l|l|}
\hline$L$ & 2 & 4 \\
\hline$L$ & 2 & 4 \\
\hline$L$ & 2 & 4 \\
\hline$I$ & 2 & 4 \\
\hline$A$ & 2 & 4 \\
\hline$L$ & 2 & 4 \\
\hline
\end{tabular}

\begin{tabular}{|l|l|l|}
\hline I & 2 & 4 \\
\hline
\end{tabular}

\begin{tabular}{|l|l|l|}
\hline A & 2 & 4 \\
\hline L & 2 & 4 \\
\hline
\end{tabular}

\begin{tabular}{|l|l|l}
$\mathrm{L}$ & 2 & \\
\hline $\mathrm{I}$ & 2 & \\
\hline
\end{tabular}

\begin{tabular}{|l|l|l|}
\hline $\mathrm{L}$ & 2 & 4 \\
\hline $\mathrm{L}$ & 2 & 4 \\
\hline
\end{tabular}

\begin{tabular}{|l|l|l|}
\hline $\mathrm{L}$ & 2 & 4 \\
\hline & 2 & 4 \\
\hline
\end{tabular}

\begin{tabular}{|l|l|l|}
\hline I. & 2 & 4 \\
\hline
\end{tabular}

\begin{tabular}{|l|l|l|}
\hline $\mathrm{L}$ & 2 & 4 \\
\hline $\mathrm{L}$ & 2 & 4 \\
\hline $\mathrm{L}$ & 2 & 4 \\
\hline
\end{tabular}

\begin{tabular}{lll}
\hline $\mathrm{L}$ & 2 \\
\hline
\end{tabular}

\begin{tabular}{|l|l|}
\hline $\mathrm{L}$ & 2 \\
\hline
\end{tabular}

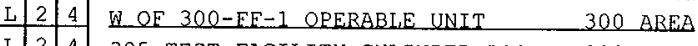
Check if information below is identical to the information submitted last

Storage codes and Loca
(Non-Confidential)
Storage Locations

\section{$=$}

CONEX BOX E OE 242A EVAPORATOR. $242 \mathrm{AC}$ SHOP

2620W SHOP

$27075 X$ SHOP

2711 E GARAGE/SHOP

$2211 \mathrm{E}$ GARAGE/SHOP

271B BUILDING

271B BUILDTNG

2721 EA BUILDING.

272AW BOTTLE DOCK

BOTTLE DOCK E OF $272 \mathrm{WA}$

27347K GAS STOBAGE

$200 E$ AREA $200 E$ ABEA 200W AREA 200W AREA 200E AREA 20OE AREA

\section{$275 E$ CYLINDER DOCK} $200 E$ AREA $200 E$ AREA $200 E$ AREA $200 E$ AREA $200 W$ AREA 200W AREA

\section{W SHOP} $200 E$ AREA $277 \mathrm{~W}$ CYLINDER DOCK 200W AREA 284W STEAM PLANT, DEACTIVATED 200W AREA 305 TEST EACYIITY CYLINDER DOCK 300 AREA I certify under penalty of law that I have personally examined and am familiar with the information submitted in pages one through 70 inquiry of those individuals responsible for obtaining the information, I believe that the submittedinformation is tree, accyrate, and complete.

James E. Rasmussen, Director Environmental Assurance, Permits and Policy Division Name and official title of owner/operator OR owner/operator's authorized representative

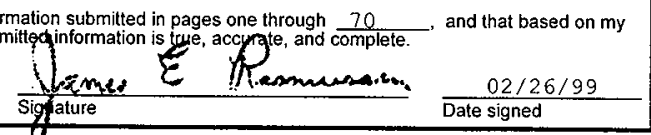
Date signed

\begin{tabular}{|l} 
Optional Attachments \\
$\mathrm{X}$ \\
\hline $\begin{array}{l}\text { I have attached a site plan } \\
\text { I have attached a list of site } \\
\text { coordinate abbreviations } \\
\text { I have attached a description of } \\
\text { dikes and other safeguard measures }\end{array}$ \\
A-6000-633 (02/98)
\end{tabular}


Washington Community Right-To-know \# :

\section{TIER TWO}

EMERGENCY

AND

HAZARDOUS

CHEMICAL

INVENTORY

Specific

Information:

Facility Identification

Name U. S. DERARTMENT OF ENERGY - HANEORD SITE

Street 825 JADWLN AVENUE

City RICHLAND _ County BENTON State WA Zip 99352

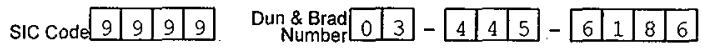

\begin{tabular}{|c|c|}
\hline \multirow{2}{*}{$\begin{array}{l}\text { For } \\
\text { Official } \\
\text { Usie } \\
\text { Oniy }\end{array}$} & $10 \#$ \\
\hline & Date \\
\hline
\end{tabular}

WA7890008967

Page 44 of 70 Ownei/Operator Name

Name $U . S$. DEPARTMENT OF ENERGY

Mail Address P.O. BOX 550, RICHLAND WA 99352

Phone (509) 376-7411 Emèrgency Contact

Name PATROL OPERATIONS CENTER

Title SHIFT COMMANDER Phone $(509) \quad 373-3800$ $24 \mathrm{Hr}$. Phone (509) 373-3800

Name

Title

Phone (1) 24. Hr. Phone
Important: Read all instructions before completing form Chemical Description

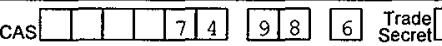
Chem. Name PRORANE

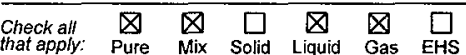
EHS Name

CAS \begin{tabular}{l|l|l|l|l|l|l|l|l|}
\hline & & & & 7 & 4 \\
\hline
\end{tabular}

Chem. Name PROPANE.

\begin{tabular}{lllllll}
\hline Checkall & $\bigotimes$ & $\bigotimes$ & $\square$ & $\bigotimes$ & $\square$ & $\square$
\end{tabular}
that apply: Pure Mix Solid Liquid Gas EHS

EHS Name

\begin{tabular}{|l|l|l|l|l|l|l|l|l|}
\hline CAS & & & & & 7 & 4 \\
\hline 9
\end{tabular}

Chem. Name PRQPANE

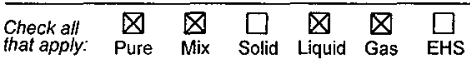

EHS Name

Certification (Read and sign after completing all sections).

I certify under penalty of law that I have personally examined and am familiar with the information submitted in pages one through 70

inquiry of those individuals responsible for obtaining the information, I believe that the submittoginformation is trye, accyrate, and complete.

James E. Rasmussen, Director EnvironmentaI

Assurance, Permits and Policy Division

Name and official title of owner/operator OR owner/operator's authorized representative
Reporting Period: From January 1 to December 31, 1998

\section{Physical}

(Check bll that apply)

\begin{tabular}{|c|c|}
\hline & Fire \\
\hline$x$ & $\begin{array}{l}\text { Sudden Release } \\
\text { of Pressure }\end{array}$ \\
\hline & Reactivity \\
\hline & Immediate (acute) \\
\hline & Delayed (chronic \\
\hline
\end{tabular}

$\mathrm{X}$ Fire

$\mathrm{X}$ Sudden Release

of Pressure

$\mathrm{X}$ Immediate (acute)

$\mathrm{X}$ Delayed (chronic)

\begin{tabular}{|l|l|}
\hline $\mathrm{X}$ & $\begin{array}{l}\text { Fire } \\
\mathrm{x}\end{array}$ \\
$\begin{array}{l}\text { Sudden Release } \\
\text { of Pressure } \\
\text { Reactivity }\end{array}$ \\
\hline
\end{tabular}

Reactivity

$X$ immediate (acute)

$\mathrm{x}$ Delayed (chronic)

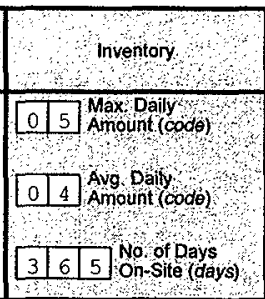

\begin{tabular}{|l|l|l|l|l}
\hline 0 & 5 & Max Dally
\end{tabular}

\begin{tabular}{|l|l|l|l|l|}
\hline 0 & 4 & Alvg Daily \\
\hline
\end{tabular}

\begin{tabular}{|l|l|l|l}
\hline 3 & 6 & 5 & $\begin{array}{c}\text { No of Days } \\
\text { On Site (days) }\end{array}$ \\
\hline
\end{tabular}

\begin{tabular}{|l|l|}
\hline 0 & 5 \\
\hline
\end{tabular}

\begin{tabular}{|l|l|l|l|l|l}
\hline 0 & 4 & Avg Daily \\
\hline
\end{tabular}

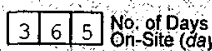

y $\mathrm{r}$

es $\mathrm{s}$

\begin{tabular}{|l|l|l|l|l|l|l|}
\hline $\mathrm{L}$ & 2 & 4 \\
\hline
\end{tabular}

\begin{tabular}{l|l|l}
$\mathrm{L}$ & 2 & 4 \\
\hline
\end{tabular}

\begin{tabular}{llll}
$L$ & 2 & 4 & 324 \\
\hline & & BUIIDING GAS DOCK
\end{tabular}

\begin{tabular}{llll}
$\mathrm{L}$ & 2 & 4 & 328 SHOP \\
\hline
\end{tabular}

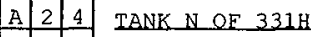

\begin{tabular}{l|l|l|}
\hline $\mathrm{L}$ & 2 & 4 \\
\hline
\end{tabular}

\begin{tabular}{|l|l|l|}
\hline L & 2 & 4 \\
\hline
\end{tabular}

\begin{tabular}{lllll}
\hline$L$ & 2 & 4 & 3722 & $3 H O P$ \\
\hline
\end{tabular}

\begin{tabular}{lll}
$L$ & 2 & 4 \\
\hline
\end{tabular}

$\begin{array}{llll}\mathrm{L} & 2 & 4 & \\ \mathrm{y} & 4704 \mathrm{~S} \text {. EIRE STATION }\end{array}$

\begin{tabular}{|lll|l}
\hline $\mathrm{L}$ & 2 & 4 & $4713 \mathrm{~B}$ SHOP \\
\hline & &
\end{tabular}

\begin{tabular}{|l|l|l|l}
\hline$L$ & 2 & 4 & $4713 D$ \\
\hline
\end{tabular}

\begin{tabular}{|l|l|l|l|l|l}
\hline $\mathrm{L}$ & 2 & 4 & $4722 \mathrm{C}$ & SHOP \\
\hline
\end{tabular}

\begin{tabular}{l|l|l|l|l}
\hline$A$ & 2 & 4 & \\
\hline
\end{tabular}

\begin{tabular}{|l|l|l|l}
\hline$L$ & 2 & 4 & TRAILER AT 6090 \\
\hline & & & 4
\end{tabular}

\begin{tabular}{llll}
$\mathrm{L}$ & 2 & 4 & 6092 LOADING DOCK \\
\hline & & 4
\end{tabular}

\begin{tabular}{l|l|l|}
$\mathrm{A}$ & 2 & 4 \\
\hline & & \\
\hline
\end{tabular}

6092 LOADING DOCK

IANK AT 6092N GAS STORAGE HAMMER EACIIITY $609 A$. EIRE STAT RT 3 BETWEEN 200E $/ \mathrm{W}$ AREAS
300 AREA 300 AREA 300 AREA

300 AREA 300 AREA 300 AREA $\square$ 300 AREA 300 AREA 300 AREA 400 AREA $400 \quad A R E A$ 400 AREA 400 AREA CORNER OF RT 1 \& $4 \mathrm{~N}$ HAMMER EACILITY HAMMER EACILITY Optional Attachments

and that based on my

$02 / 26 / 99$

$\mathrm{X}$ I have attached a site plan I have attached a list of site coordinate abbreviations

I have attached a description of dikes and other safeguard measures A-6000-633 (02/98) 
Washington Community Right-To-know \#:

\begin{tabular}{|c|c|}
\hline $\begin{array}{l}\text { TIER TWO } \\
\text { EMERGENCY } \\
\text { AND } \\
\text { HAZARDOUS }\end{array}$ & $\begin{array}{l}\text { Facility Identification } \\
\text { Name U. S. DERARTMENT OE ENERGY - HANEORD SITE } \\
\text { Street } 825 \text { JADWIN AVENUE } \\
\text { City RICHLAND COunty BENTON State WA Z Zip } 99352\end{array}$ \\
\hline CHEMICAL & 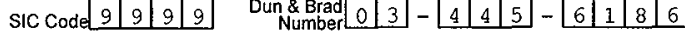 \\
\hline & $\begin{array}{l}\text { For } \\
\text { Official }\end{array}$ \\
\hline $\begin{array}{l}\text { Information } \\
\text { by Chemical }\end{array}$ & Only [Date \\
\hline
\end{tabular}

Page 45 of 70 Owner/Operator Name

Name_U, S, DERARTMENT OF ENERGY Phone (509) 376-7411 Mail Address P. O. BOX 550, RICHLAND WA 92352 Emergency Contact

Name PATROL OPERATIONS CENTER.

Title SHIFT COMMANDER

Phone (509) 373-3800 $24 \mathrm{Hr}$. Phone $(509) \quad 373-3800$

Name

Title

Phone $(1) 24 \mathrm{Hr}$. Phone $(1)$

Important: Read all instructions before completing form

Chemical Description

\section{Physical and Health (check all that apply)}

Reporting Period: From January 1 to December 31, 1998

\begin{tabular}{|l|l|l|l|l|l|l|l|l|l|l|l|l|}
\hline CAS & & & & & 7 & 4 & 8 \\
\hline
\end{tabular}

Chem. Name PRORANE

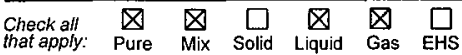

EHS Name

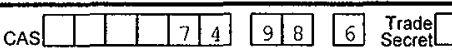

Chem. Name PROPANE

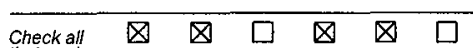

that apply: Pure Mix Solid Liquid Gas EHS

EHS Name

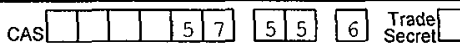

Chem. Name PROPYIENE GLYCOI,

Check all $\quad$ 邓 $\quad$ \ $\square \quad \square \quad \square \quad \square$

that apply: Pure Mix Solid Liquid Gas EHS

EHS Name

Certification (Read and sign after completing all sections)

I certify under penalty of law that I have personally examined and am familiar with the information submitted in pages one through 70 inquiry of those individuals responsible for obtaining the information, I believe that the submitted information is true, accurate, and complete.

James E. Rasmussen, Director Environmental Assurance, Permits and Policy Division Name and official title of owner/operator OR owner/operator's authorized representative

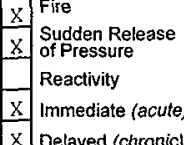

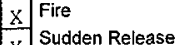

$\mathrm{X}$ of Pressure

Reactivity

$X$ immediate (acute)

$\mathrm{X}$ Delayed (chronic)

\begin{tabular}{|l|l|}
\hline & $\begin{array}{l}\text { Fire } \\
\text { Sudden Release } \\
\text { of Pressure } \\
\text { Reactivity }\end{array}$ \\
\hline $\mathrm{X}$ & $\begin{array}{l}\text { Immediate (acute) } \\
\text { Delayed (chronic) }\end{array}$ \\
\hline
\end{tabular}

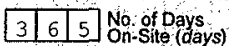
\begin{tabular}{|l|l|l|}
\hline 0 & 4 \\
\hline
\end{tabular} Max Daily \begin{tabular}{|l|l|l|l}
\hline 0 & 4 & Avg Daily
\end{tabular}

3765 No. of obays $\begin{array}{ll}0 & 4 \\ 0\end{array}$ Avg. Daily $(\operatorname{cod} \theta)$

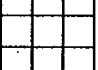

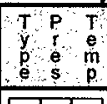

\section{\begin{tabular}{ll|l|l}
\hline$L$ & 2 & 4 \\
\hline
\end{tabular}} \begin{tabular}{|l|l|l|}
\hline $\mathrm{I}$ & 2 & 4 \\
\hline
\end{tabular}

\begin{tabular}{|l|l|l|}
\hline$I$ & 2 & 4 \\
\hline
\end{tabular}

\begin{tabular}{|l|l|l|}
\hline $\mathrm{I}$ & 2 & 4 \\
\hline
\end{tabular}

\begin{tabular}{|l|l|l|}
\hline I & 2 & 4 \\
\hline
\end{tabular}

\begin{tabular}{|l|l|l|}
\hline $\mathrm{I}$ & 2 & 4 \\
\hline
\end{tabular}

\begin{tabular}{|l|l|l|}
\hline $\mathrm{L}$ & 2 & 4 \\
\hline
\end{tabular}

\begin{tabular}{ll|l|l|}
\hline A & 2 & 4 \\
\hline
\end{tabular}

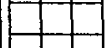

\begin{tabular}{|l|l|l|}
\hline$D$ & 1 & 4 \\
\hline$N$ & 1 & 4 \\
\hline
\end{tabular} \begin{tabular}{|l|l|l|}
\hline $\mathrm{N}$ & 1 & 4 \\
\hline & & \\
\hline
\end{tabular} \begin{tabular}{lll|l|}
\hline$E$ & 1 & 4 \\
\hline
\end{tabular} \begin{tabular}{l|l|l|}
\hline $\mathrm{D}$ & 1 & 4 \\
\hline & 1 & 4 \\
\hline
\end{tabular} \begin{tabular}{lll}
$\mathrm{N}$ & 1 & 4 \\
\hline $\mathrm{E}$ & 1 & 4 \\
\hline
\end{tabular} 2025E_BUILDING, ROOM 112

Storage Locations 6265 I.AB

\section{Storage Coces and Locations
(Non-Confidential)}

609A FIRE STAT RT 3 BETWEEN 200E/W AREAS N2R I,AB, ROOM F NE OF 200W AREA $E$ OF 200W AREA W OE 200E AREA HTS PIPEYARD BOTTIE RACK NE OE 200E ABEA 100N AREA 20OE ABEA $+2$

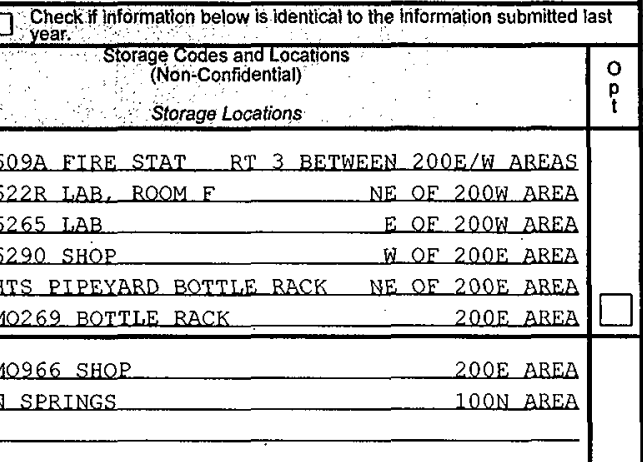
$1171 \mathrm{C}$ STORAGE SHED, DEACTIVATED 1100 ABEA 1900R WAREHOUSE 100D/DR AREA IOOK AREA $100 K$ AREA 200E AREA

o
$\mathbf{p}$
$\mathbf{t}$




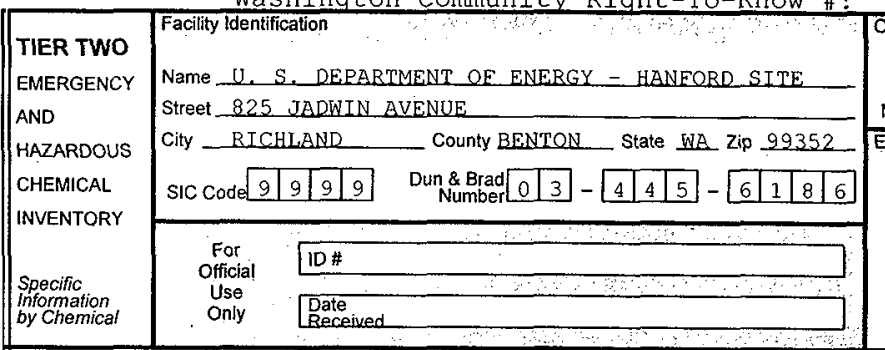

Reporting Period: Fr
Physical
and Health
Hazards
Check all (hat apply)
Fire

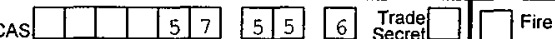
CAS 1. Chem. Name PROPYLENE GI,YCOI,

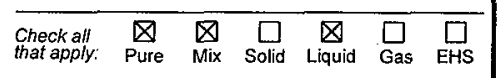
EHS Name \begin{tabular}{l|l|l|l|l|l|l|l|l|l|l|l|l|l|l|}
\hline CAS & & & & 5 & 7 & 5 & 6 \\
\hline
\end{tabular} Chem. Name PBOPYLENE GIYCOL

$\begin{array}{lllll}\text { Checkall } & \bigotimes & \bigotimes & \square & \square \\ \text { that apply: } & \square & \square & \square \\ \text { Pure } & \square\end{array}$
EHS Name

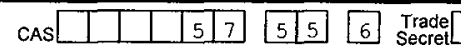
Chem. Name PROPYIENE GLYCOL

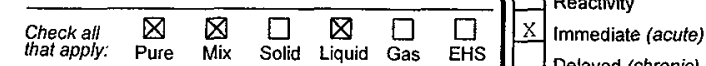
Lelayed (chronic)

\begin{tabular}{|l}
\hline $\mathrm{x}$ \\
$\mathrm{x}$ \\
$\mathrm{S}$ \\
of Prden Release \\
Reactivity \\
Immediate (acute) \\
Delayed (chronic)
\end{tabular}

\section{Fire}

Sudden Release -1 of Pressure

Reactivity $\mathrm{X}$ Immediate (acute) Delayed (chronic)

\begin{tabular}{|c|c|}
\hline & Fire \\
\hline & $\begin{array}{l}\text { Sudden Release } \\
\text { of Pressure }\end{array}$ \\
\hline & Reactivity \\
\hline$x$ & Immediate (acute) \\
\hline & Delayed (chronic) \\
\hline
\end{tabular}

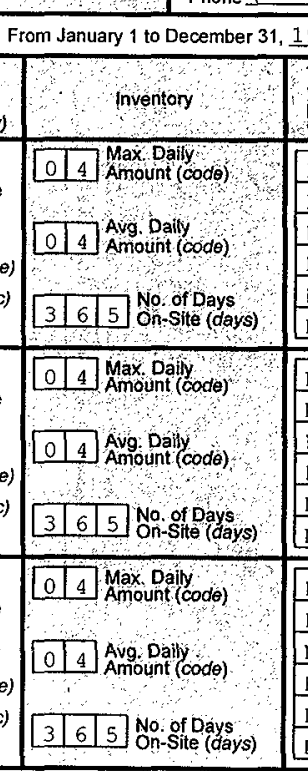

Name PATROY, OPERATIONS CENTEB Phone (509) 373-3800

Name

Name
Title SHIFT COMMANDER $24 \mathrm{Hr}$. Phone (509) 373-3800

Title 24 Hr. Phone ( )

\section{EHS Name}

Certification (Read and sign after completing all sections).

II certify under penalty of law that I have personally examined and am familiar with the information submitted in pages one through 70 inquiry of those individuals responsible for obtaining the information, I believe that the submitted information is true accurate and complete. James $\mathrm{E}$. Rasmussen, Dixector Environmental
incliry of those individuals responsible for obtaining the information, l bel Assurance, Permits and Policy Division iName and official title of owner/operator OR owner/operator's authorized representative

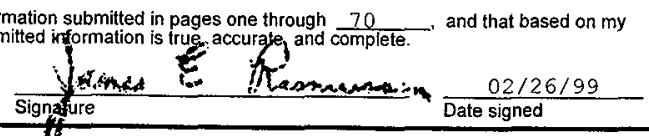
Check
year:

$T P$
$y$ T
pe
s

\section{Storage Codes and Locations
(Non-Confidential)} Storage Locations

\begin{tabular}{|c|c|c|c|c|}
\hline$\underline{F}$ & 1 & 4 & CONEX BOXES E OF $2025 \mathrm{E}$ & $200 E$ AREA \\
\hline $\mathrm{F}$ & 1 & 4 & LAYDOWN YARD BY $2241 \mathrm{~B}$ & $200 \mathrm{E}$ AREA \\
\hline D & 1 & 4 & $2336 \mathrm{~W}$ BUILDING & 200W AREA \\
\hline $\mathrm{R}$ & 1 & 4 & $2336 \mathrm{~N}$ BUILDING SYSTEM & 200W AREA \\
\hline $\mathrm{D}$ & 1 & 4 & STORAGE UNIT BY $234=52$ & $200 \mathrm{~W}$ AREA \\
\hline$\underline{E}$ & 1 & $\underline{4}$ & STOBAGE UNIT BY 234-52 & $200 \mathrm{~W}$ AREA \\
\hline $\mathrm{N}$ & 1 & 4 & $234-52$ BUILDING & $200 W$ AREA \\
\hline $\mathrm{N}$ & 1 & 4 & 2362,BUILDING, CORRIDOR IW & 200W AREA \\
\hline D & 1 & 4 & STORAGE UNIT BY 241A401 & $200 E$ AREA \\
\hline D & 1 & 4 & 24182 CONTROI, ROOM & $200 \mathrm{E}$ AREA \\
\hline $\mathrm{N}$ & 1 & 4 & $2703 E$ BUILDING & 200E AREA \\
\hline $\mathrm{N}$ & 1 & 4 & 2713 WC WASHWATER RECOVERY EAC & 200W AREA \\
\hline D & 1 & $\underline{4}$ & 2721EA BUTLDING & $200 E$ AREA \\
\hline$\underline{E}$ & 1 & 4 & STORAGE UNIT BY 272AW & 200E_AREA \\
\hline $\mathrm{N}$ & 1 & 4 & $272 W$ SHOP & 200W AREA \\
\hline D & 1 & 4 & STORAGE UNIT N OE 272WA & 200W AREA \\
\hline $\mathrm{D}$ & 1. & 4 & LAYDOWN AREA BY $277 \mathrm{~A}$ SHOP & $200 \mathrm{E}$ AREA \\
\hline$\underline{D}$ & 1 & 4 & 300-FE-1 ORERABLE UNIT & $300 \quad$ AREA \\
\hline
\end{tabular}
Optional Attachments coordinate abbrevialions I have attached a description of dikes and other safeguard measures 
Washington Community Right-To-know \#:

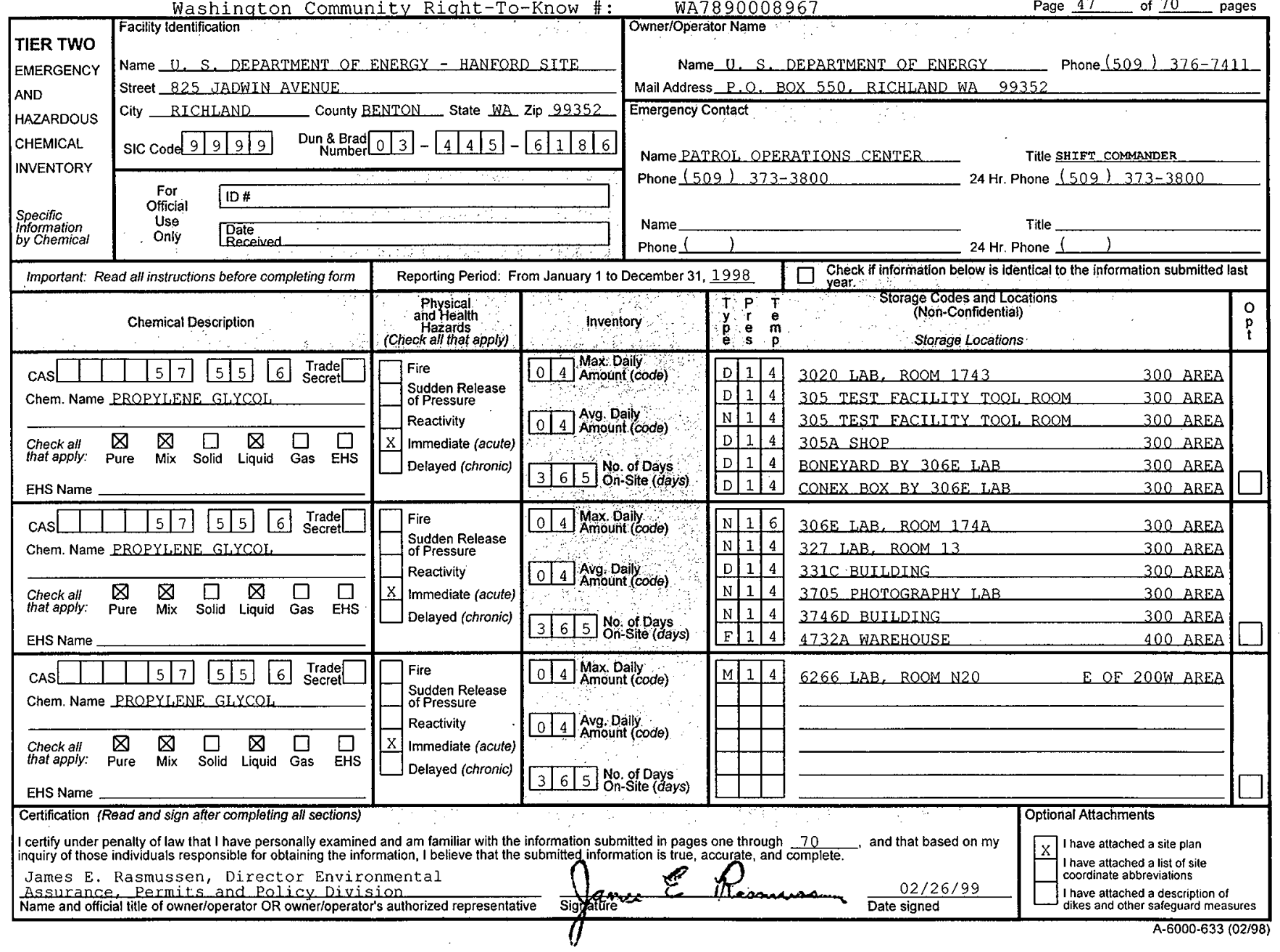


Washington Community Right-To-know \#:

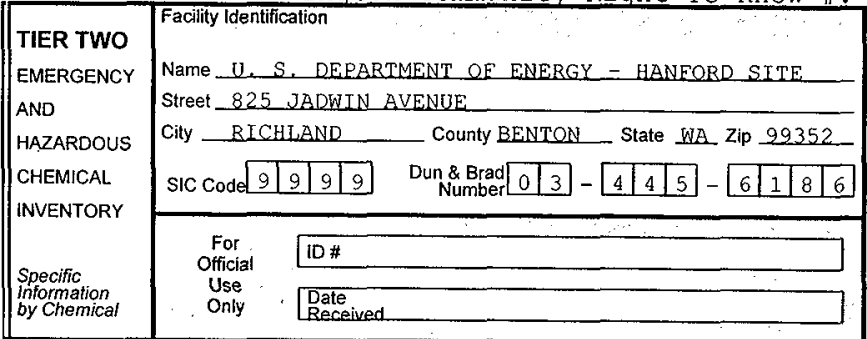

WA7 890008967 Owner/Operator Name

Name_U.S. DERARTMENT OF ENERGY

Mail Address P.Q. BOX 550, RICHLAND WA 9935 Emergency Contact

Name PATROL OPERATIONS CENTER

Phone $(509) \quad 373-3800$.

Title SHIFT COMMPNDER $24 \mathrm{Hr}$. Phone (509) 373-3800

Name

Phone $(1)$

Title 24 Hr. Phone (1)

Phone (509) $376-7411$

Reporting Period: From January 1 to December 31, 1998

\section{Physical}

and Health (Check all that apply)

Chem. Name SILICA, CRYSTALI,INECRISTOBALTTE $\begin{array}{lllll}\text { Check all } & \square & \square & \square & \square \\ \text { that apply: } & \square & \square & \square \\ \text { Mix } & \square & \square\end{array}$

EHS Name

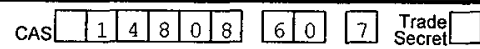
Chem. Name SIIICA, CRYSTALLINEQUARTZ

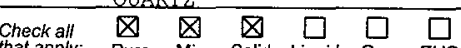

that apply: Pure Mix Solid Liquid Gas EHS

EHS Name

CAS \begin{tabular}{l|l|l|l|l|l|}
\hline & 1 & 4 & 8 & 0 & 8 \\
\hline 6 & 0 \\
\hline
\end{tabular}$\quad$\begin{tabular}{ll|l|l|}
\hline Trade \\
\hline
\end{tabular}

Chem. Name SILICA, CRYSTALIINEQUARTZ

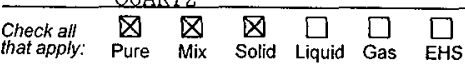

EHS Name

Certification (Read and sign after completing all sections) II certify under penalty of law that I have personally examined and am familiar with the information submitted in pages one through 70
iinquiry of those individuals responsible for obtaining the information, I believe that the submitteg formation is true, accurate, and comp

James E. Rasmussen, Director Environmental

Assurance, Permits and Policy Division

iName and official title of owner/operator OR owner/operator's authorized representative

\section{Fire}

of Pressure

Reactivity

Immediate (acute)

Delayed (chronic)

\section{$\longrightarrow$ Fire}

Sudden Release of Pressure

Immediate (acute)

$\mathrm{X}$ Delayed (chronic)

\begin{tabular}{l}
$\square$ \\
$\square$ \\
\hline Sudden Release \\
of Pressure \\
Reactivity \\
Immediate (acute) \\
Delayed (chronic)
\end{tabular}

Delayed (chronic)
CAS \begin{tabular}{|l|l|l|l|l|l|l|l|l|}
\hline & 1 & 4 & 6 & 6 & 4 \\
\hline
\end{tabular}

Reactivity

\begin{tabular}{|c|c|c|}
\hline & & Inventory \\
\hline 0 & 5 & $\begin{array}{l}\text { Max. Daily } \\
\text { Amount (codo) }\end{array}$ \\
\hline 0 & 5 & $\begin{array}{l}\text { Avg Daily. } \\
\text { Ámount }(\operatorname{cod} \theta)\end{array}$ \\
\hline 3 & 6 & 5 No. of Days \\
\hline 0 & 5 & $\begin{array}{l}\text { Max Dajly } \\
\text { Amount (code) }\end{array}$ \\
\hline 0 & 5 & $\begin{array}{l}\text { Avg. Daily } \\
\text { Amount }(\operatorname{cod} \theta)\end{array}$ \\
\hline 3 & 6 & 5 No of Days \\
\hline 0 & 5 & $\begin{array}{l}\text { Max Daily } \\
\text { Amount (code) }\end{array}$ \\
\hline 0 & 5 & Avg. Daily \\
\hline 3 & 6 & 5 No. of Days \\
\hline
\end{tabular}

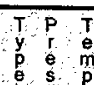

\begin{tabular}{l|l|l|l|}
\hline T & 1 & 4 \\
\hline
\end{tabular}

\begin{tabular}{|l|l|l|}
\hline $\mathrm{J}$ & 1 & 4 \\
\hline $\mathrm{J}$ & 1 & 4 \\
\hline
\end{tabular}

\begin{tabular}{l|l|l|l|}
\hline $\mathrm{J}$ & 1 & 4 \\
\hline
\end{tabular} $\square$. Check

\section{Storage Codes and Locations}

(Non-Confidential)

Storage Locations
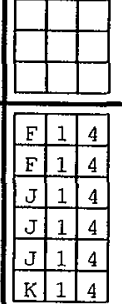

CONEX BOX NE OE 105KE

$100 \mathrm{~K} A \mathrm{ABE}$

CONEX BOX. NE OE 105KW 10OK AREA

19ODR WAREHOUSE

2101M WAREHOUSE

$221 T$ CANYON

$221 \mathrm{~T}$ CANYON

20OE AREA

RT 3 BETWEEN 200E/W AREAS

\begin{tabular}{|l|l|l|l|l|l|l|l|l|l}
\hline$N$ & 1 & $422 S$ LAB \\
\hline
\end{tabular}

$\mathrm{N} 1 \mathrm{~A}$ (202SA

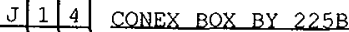

\begin{tabular}{l|l|l|l|l|l|l|l}
\hline$J$ & 1 & $433 S$ \\
\hline
\end{tabular} iName and official title of owner/operator OR owneroperator's authoized representative Si

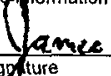
Sigg ture

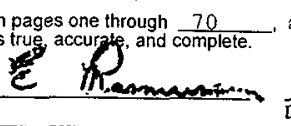
Date signed
IT BY 234-52 Optional Attachments
ON_AREA 200W AREA $200 E$ AREA $200 W$ AREA 200W AREA 200W AREA

OD/DR AREA $200 E$ ABEA 200W AREA $2004 \mathrm{~T}$

\begin{tabular}{l|l}
$A B A$ \\
$A B$ \\
$A B$
\end{tabular}

$\mathrm{x}$ I have attacned a site plan I have attached a list of site coordinale abbreviations I have attached a description of dikes and other safeguard measures A-6000-633 (02/98) 
Washington Community Right-To-know \#:

\begin{tabular}{|c|c|}
\hline $\begin{array}{l}\text { TIER TWO } \\
\text { EMERGENCY } \\
\text { AND } \\
\text { HAZARDOUS } \\
\text { CHEMICAL }\end{array}$ & 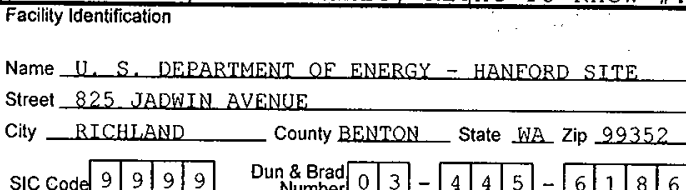 \\
\hline $\begin{array}{l}\text { ecific } \\
\text { formation } \\
\text { Chemical }\end{array}$ & $\begin{array}{ll}\begin{array}{c}\text { For } \\
\text { Official } \\
\text { Use }\end{array} & 10 \# \\
\text { Only } & \text { Date } \\
\text { Receiver }\end{array}$ \\
\hline
\end{tabular}

Mail Address__. O _ BOX 550, RICHLAND WA 99352 Emergency Contact

Name PATROL ORERATIONS CENTER

Title SHIET COMMANDER Phone (509) 373-3800 24 Hr. Phone (509) 373-3800

Name

Title

Phone (1) $24 \mathrm{Hr}$. Phone (1)

Important: Read all instructions before completing form

Reporting Period: From January 1 to December 31, 1998 Phiysical and Health Chemical Description (Check all that apply)

CAS \begin{tabular}{l|l|l|l|l|l|l|l|}
\hline & 1 & 4 & 8 & 0 & 8 \\
\hline
\end{tabular}$\quad$\begin{tabular}{ll}
\hline 7 & 0 \\
\hline
\end{tabular} $\begin{aligned} & \text { Trade } \\
& \text { Secret }\end{aligned}$ Chem. Name SILICA. CRYSTALLINEQUART2

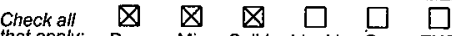
that apply: Pure Mix solid Liquid Gas EHS

EHS Name

\section{TFire}

Sudden Release of Pressure

Reactivity

immediate (acute)

$\mathrm{X}$ Delayed (chronic)

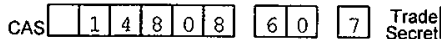
Chem. Name SILICA，CRYSTALIINEOUARTZ

\begin{tabular}{lllllll}
\hline Check all & $\bigotimes$ & $\bigotimes$ & $\bigotimes$ & $\square$ & $\square$ & $\square$
\end{tabular} that apply: Pure Mix Solid Liquid Gas EHS

EHS Name

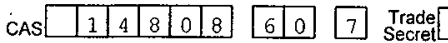
Chem. Name SIIJCA, CRYSTALLINEOUARTZ

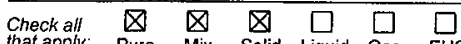
that apply: Pure Mix Solid Liquid Gas EHS

EHS Name Certification (Read and sign after completing all sections)

\section{Fire}

Sudden Release of Pressure

Reactivity

Immediate (acute)

$\mathrm{x}$ Delayed (chronic)

\section{$\square$ Fire}

Sudden Release Sudden Releas
of Pressure

Reactivity

Immediate (acute) $\mathrm{X}$ Delayed (chronic)

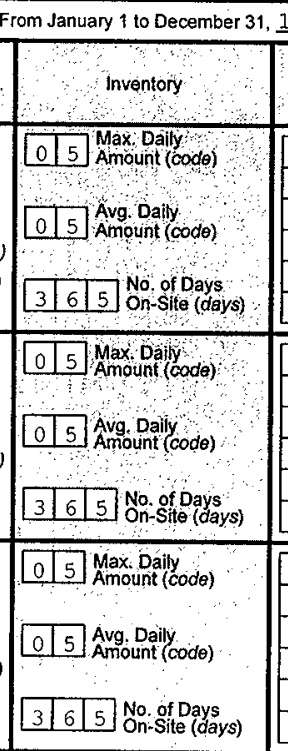

1998

$\mathrm{T}$
$\mathrm{T}$
$\mathrm{p}$
$\mathrm{e}$
$\mathrm{e}$
7 Check

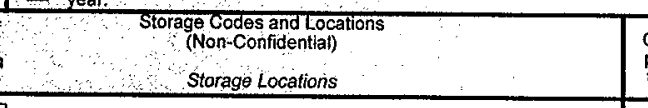

\begin{tabular}{|l|l|l|}
\hline $\mathbf{E}$ & 1 & 4 \\
\hline
\end{tabular}

234-52 BUILDING, ROOM 202 200W AREA I 14 234-52, BUIIDING, ROOM 186 200W ABEA

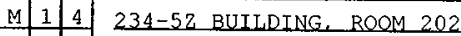
$200 W$ AREA $M \quad 1 \quad 4$ STORAGE UNIT BY 234-52 $\mathrm{N}) 1 \mathrm{4}$ 234-52 BUILDING STORAGE UNIT BY $234-52$ $200 W$ AREA \begin{tabular}{|l|l|l|}
\hline $\mathrm{N}$ & 1 & 4 \\
\hline
\end{tabular}

\begin{tabular}{|l|l|l|}
\hline$J$ & 1 & 4 \\
\hline$E$ & 1 & 4 \\
\hline$D$ & 1 & 4 \\
\hline
\end{tabular}

2715AW STOREROOM $200 W$ AREA 200W AREA 200E AREA CONEX BOXES BY 306E I,AB 300 AREA \begin{tabular}{lllll}
$\mathrm{E}$ & 1 & 4 & $3707 \mathrm{E}$ SHOP \\
\hline
\end{tabular} 300 AREA \begin{tabular}{l|l|l|}
\hline$R$ & 1 & 4 \\
\hline
\end{tabular} 300 AREA \begin{tabular}{|l|l|l|l}
\hline $\mathrm{D}$ & 1 & 4 \\
\hline $\mathrm{D}$ & & $4732 \mathrm{~A}$ WAREHOUSE & 400 AREA \\
\hline
\end{tabular} D 1146 607 BATCH PLANT BT 3. BETWEEN 200E/W AREAS

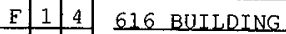
BT 3. BETWEEN 200E/W AREAS

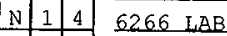
E OF 20OW AREA

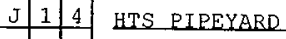
NE OE 200E AREA CONEX BOX N OF MO743 2006 AREA

I certify under penalty of law that I have personally examined and am familiar with the information submitted in pages one through 70 inquiry of those individuals responsible for obtaining the information, I believe that the submitted submitted in pages one through 70

James E. Rasmussen, Director Environmental Assurance, Permits and Policy Division Name and official title of owner/operator OR owner/operator's authorized representative

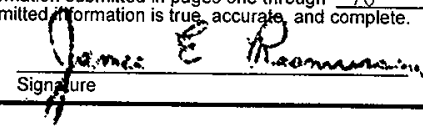
and that based on my $02 / 26 / 99$ Date signed

\section{Optional Attachments}

$x$ I have attached a site plan I have attached a list of site coordinate abbreviations I have attached a description of dikes and other safeguard measures 


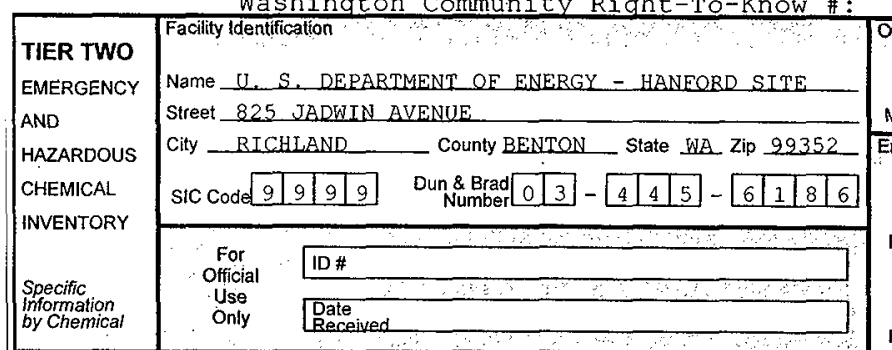

Name PATROL OPERATIONS CENTER Phone $(509) \quad 373-3800$

Title SHIFT COMMANDER

Name Phone 1 hone $(509) \quad 373-3800$

Title $24 \mathrm{Hr}$. Phone $(1)$
Important: Read all instructions before completing form

Chemical Description

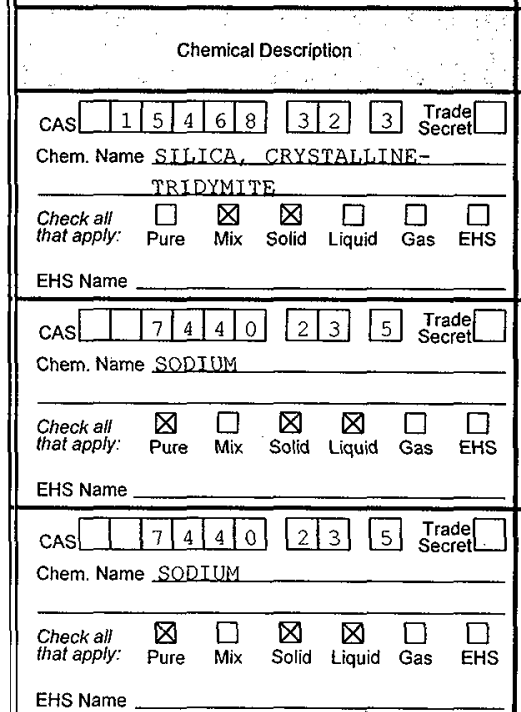

Reporting Period: From January 1 to December 31, 1998

Physical
and Health
Hazards
(Check all that apply)

\begin{tabular}{|c|c|}
\hline \multicolumn{2}{|r|}{ Fire } \\
\hline & $\begin{array}{l}\text { Sudden Refease } \\
\text { of Pressure }\end{array}$ \\
\hline & Reactivity \\
\hline & sute) \\
\hline$x$ & Del \\
\hline
\end{tabular}

\section{$\mathrm{x}$ Fire}

Sudden Release -1 of Pressure

$x$ Reactivity

$\mathrm{X}$ Immediate (acufe) Delayed (chronic)

\section{$x$ Fire Sudden Release of Pressure Reactivity $\mathrm{X}$ Immediate (acute) Delayed (chronic)}

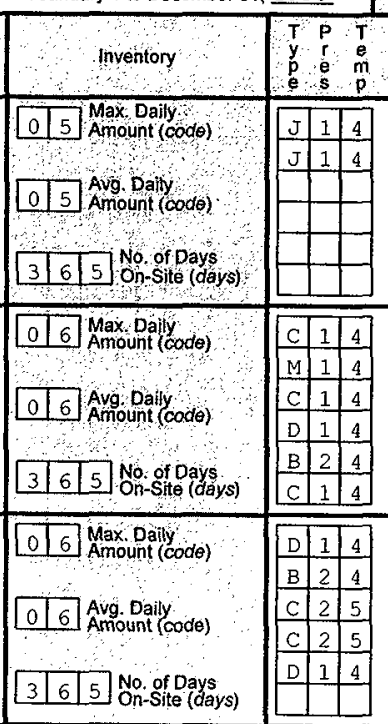

Certification (Read and sign after completing all sections)

I certify under penalty of law that I have personally examined and am familiar with the information submitted in pages one through 70 inquiry of those individuals responsible for obtaining the information, I believe that the submittedinformation is true, accurate, and complete. James E. Rasmussen, Director Environmental Assurance, Permits and Policy Division Name and official tille of owner/operator OR owner/operator's authorized representative

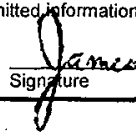

\section{Check}

-

Storage Codes and Locations
(Non-Confidential)
Storage Locations

2101M WAREHOUSE 616 BUILDING 200E ABEA RT 3.BETWEEN 200E/W AREAS

$221 T$ HEAD END $200 W$ AREA 234-52 BUILDING. BOOM 186 2727W STORAGE FACILITY 200W ABEA 335 SODIUM TEST FACILITY 300 AREA $337 \mathrm{~B}$ HIGH $-\mathrm{BAY}$ $337 \mathrm{~B}$ HIGH-BAY 300 AREA 300 AREA $\square$ $337 \mathrm{~B}$ HIGH $-\mathrm{BAY} \quad 300 \mathrm{AREA}$ UNDERGROUND TANK BY 3718M 300 AREA 403 FUELS STORAGE FACILITY 405_REACTOR BUILDING. 400 ARE:A ALKALI METAL STORAGE MODULES 200 


\section{TIER TWO}

EMERGENCY

AND

HAZARDOUS

CHEMICAL

INVENTORY

Specific

Infomation
Name U. S. DERABUMENT OF ENERGY - HANFOBD SITE

Street 825 JADWIN_AVENUE

City BICHLAND County BENTON

State WA Zip 29352

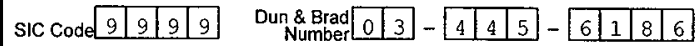

$\begin{array}{ll}\text { For } \\ \text { Official } \\ \text { Use } \\ \text { Only } & \text { ID \# } \\ & \text { Date } \\ \text { Received } & \end{array}$

Owner/Operator Name

Name_U. $S$, DEPARTMENT OF ENERGY

Phone (509) 376-7411

Mail Address_. P.O. BOX 550, RICHLAND WA 99352

Emergency Contact

Name PATROI, OPERATIONS CENTER

Phone (509) 373-3800

Title SHIFT CONANDER

Name

Phone 1 $24 \mathrm{Hr}$. Phone $24 \mathrm{Hr}$. Phone $(509) 373-3800$

Important: Read all instructions before completing form

Chemical Description

Reporting Period: From January 1 to December 31, 1998

Physical
and Health (Check all that appily)

CAS \begin{tabular}{l|l|l|l|l|l|l|l|l|}
\hline & & 7 & 6 & 4 & 7 & 1 & 4 \\
\hline
\end{tabular}

Chem. Name SODIUM CHLORTDE.

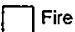

Sudden Release of Pressure

Reactivity

$\begin{array}{llllll}\begin{array}{c}\text { Check all } \\ \text { that apply: }\end{array} \underset{\text { Pure }}{\bigotimes} & \underset{\text { Mix }}{\bigotimes} & \underset{\text { Solid }}{\bigotimes} & \underset{\text { Liquid }}{\bigotimes} & \square & \square \\ \text { Gas } & \square\end{array}$ EHS Name

CAS \begin{tabular}{|l|l|l|l|l|l|l|}
\hline & & 7 & 6 & 4 & 7 \\
\hline
\end{tabular} Chem. Name SODIUM CHLORIDE

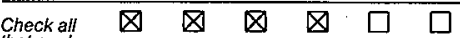
that apply: Pure Mix Solid Liquid Gas EHS

EHS Name

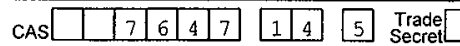

Chem. Name SODIUM CHIORIDE:

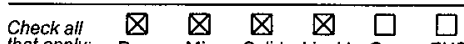

that apply: Pure Mix Solid Liquid Gas EHS

EHS Name

Certification (Read and sign after complefing all sections)

Delayed (chronic)

\begin{tabular}{|l|l}
$\square$ & $\begin{array}{l}\text { Fire } \\
\text { Sudden Release } \\
\text { of Pressure } \\
\text { Reactivity }\end{array}$ \\
$\mathrm{X}$ & $\begin{array}{l}\text { immediate (acute) } \\
\text { Delayed (chronic) }\end{array}$
\end{tabular}

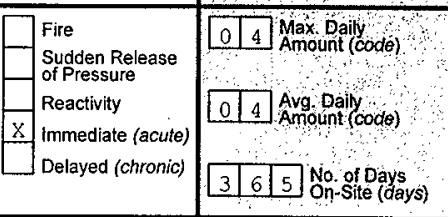

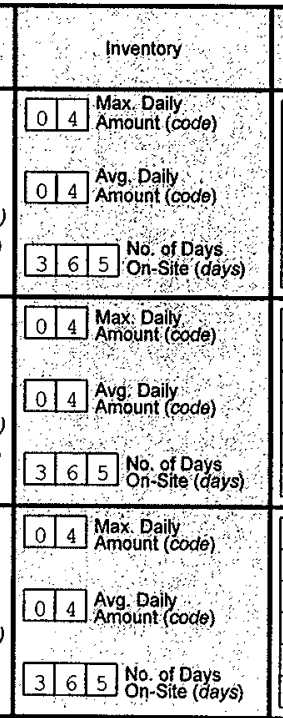

\begin{tabular}{|l|l|l|}
\hline & & \\
\hline & & \\
\hline$A$ & 1 & \\
\hline$M$ & & S \\
\hline$N$ & 1 & 4 \\
\hline$M$ & 1 & 4 \\
\hline$N$ & 1 & 4 \\
\hline$E$ & 1 & 4 \\
\hline$F$ & 1 & 4 \\
\hline$E$ & 1 & 4 \\
\hline$E$ & 1 & 4 \\
\hline$E$ & 1 & 4 \\
\hline$B$ & 1 & 4 \\
\hline$B$ & 1 & 4 \\
\hline$E$ & 1 & 4 \\
\hline$N$ & 1 & 4 \\
\hline$M$ & 1 & 4 \\
\hline$M$ & 1 & 4 \\
\hline$N$ & 1 & 4 \\
\hline$I$ & 1 & 4 \\
\hline
\end{tabular}
Check
year.

\begin{tabular}{|c|c|c|}
\hline $\begin{array}{c}\text { Storage Codes and Locations } \\
\text { (Non-Confidential) } \\
\text { Storage Locations }\end{array}$ & & $\begin{array}{l}0 \\
p \\
1\end{array}$ \\
\hline 100-DR-1_OPERABIE UNIT & $100 D / D R$ AREA & \\
\hline $105 \mathrm{NB}$ SHOP. & $100 N$ AREA & \\
\hline $105 \mathrm{NB}$ SHOP & $100 N$ AREA & \\
\hline $1143 \mathrm{~N}$ SHOP & $100 \mathrm{~N} \_\mathrm{AREA}$ & \\
\hline $165 \mathrm{KE}$ POWER CONTROL BUILDING & $100 K$ AREA & \\
\hline 1714N STORAGE BUIJDING & $100 \mathrm{~N}$ AREA & \\
\hline STORAGE UNIT SW OF $1724 \mathrm{~K}$ & $100 K$ AREA & \\
\hline $183 \mathrm{~N}$ EILTER ELANT & $100 N$ AREA & \\
\hline 183N EILTER PLANT & 100N AREA & \\
\hline $190 \mathrm{KE}$ WAREHOUSE & 10OK AREA & \\
\hline 200-2P-1 PUMP \& TREAT & 200W AREA & \\
\hline $200-2 P-2$ ERA. & 200W ABEA & \\
\hline $2025 \mathrm{E}$ BUILDING, ROOM 112 & $200 E$ AREA & \\
\hline 2025E_BUILDING, BOOM 112 & $200 E$ AREA & \\
\hline $222 \mathrm{~S} \quad \mathrm{I} A \mathrm{AB}$ & 200W AREA & \\
\hline $222 \mathrm{SA} \perp \mathrm{AB}$ & 200W AREA & \\
\hline $222 S A \_\mathrm{LAB}$ & 200W AREA & \\
\hline $234-57$ BUILDING, BOOM 202 & 200W AREA & \\
\hline
\end{tabular}

I certify under penalty of law that I have personally examined and am familiar with the information submitted in pages one through 70

inquiry of those individuals responsible for obtaining the information, I believe
James E. . Rasmussen, Di rector of Environmental

Assurance, Permits and Policy Division

Name and official title of owner/operator OR owner/operator's authorized representative and that based on my

$02 / 26 / 99$ Date signed
Optional Attachments
$\mathrm{X}$
$\begin{aligned} & \text { I have attached a site plan } \\ & \text { I have attached a list of site } \\ & \text { coordinate abbreviations } \\ & \text { i have attached a description of } \\ & \text { dikes and other safeguard measures }\end{aligned}$

A-6000-633 (02/98) 


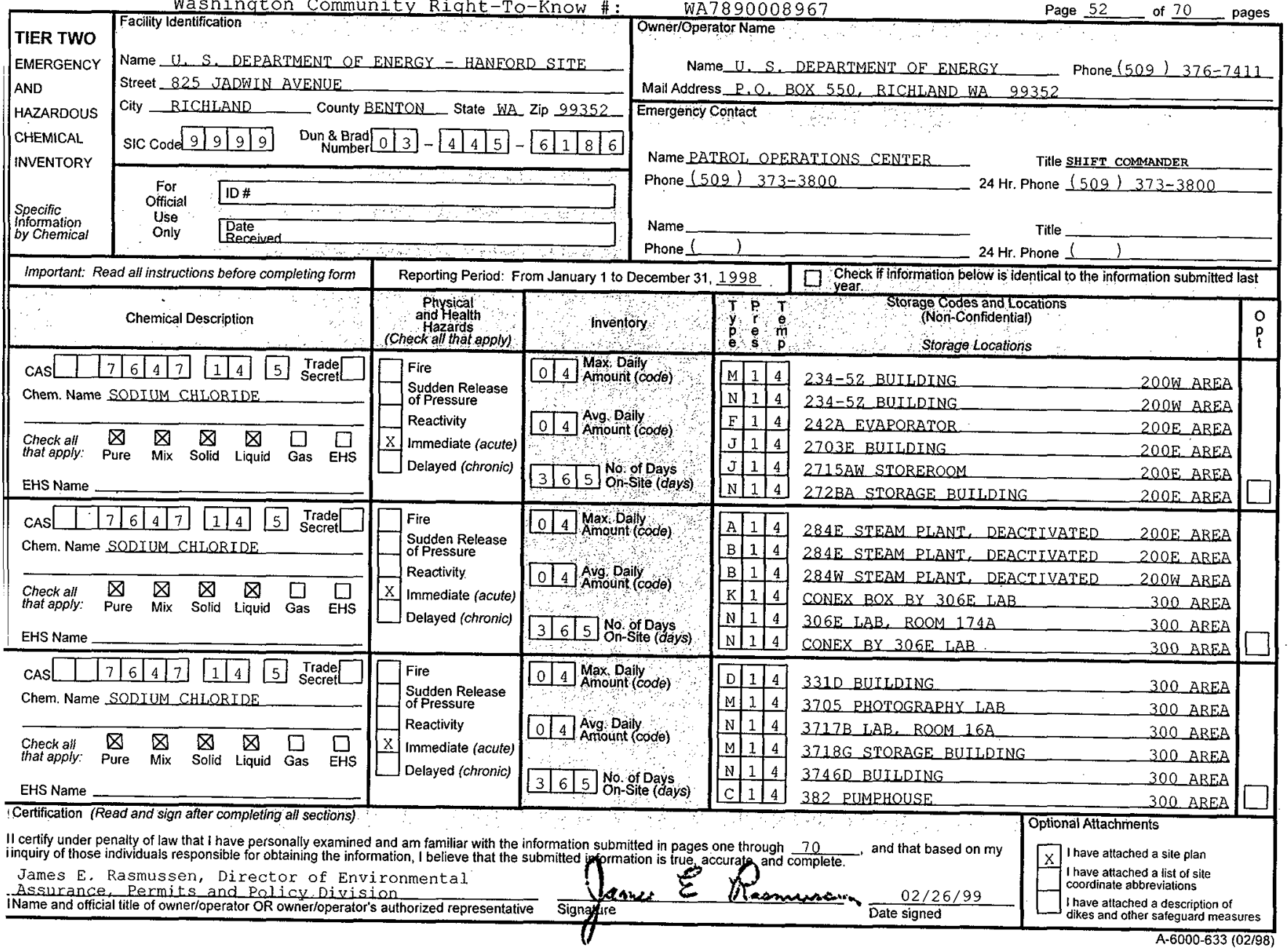


Washington Community Right-To-know \#: Facility Identification

Name U. S. DEPARTMENT OF ENERGY - HANFORD SITE Street 825 JADWIN AVENUE

EMERGENCY AND MAZARDOUS CHEMICAL INVENTORY City - RICHLAND County BENTON State WA. Zip 99352

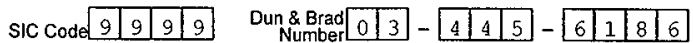
Por

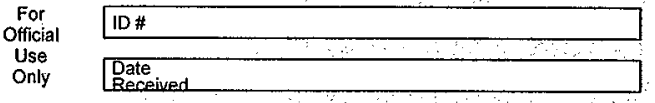

WA7890008967 Owiner/Operator Name

Name U. S. DEPARTMENT OF. ENERGY Page 53 of 70 - pages Mail Address_P.O. BOX 550, RICHLAND WA 99352

Phone $(509) \quad 376-7411$ Emergency Contact

Name PATROL OPERATIONS CENTER Phone (509) 373-3800

Name

Phone
Title SHIFT COMANDER $24 \mathrm{Hr}$. Phone $(509) \quad 373-3800$

Title $24 \mathrm{Hr}$. Phone ( )
Reporting Period: From January 1 to December 31, 1998

(C)

Physical
and. Health and. Health
Hazards (Check all that apply)
Chemical Description

\begin{tabular}{l|l|l|l|l|l|l|l|l|}
\hline CAS & & 7 & 6 & 4 & 7 \\
\hline 1 & 4 \\
\hline
\end{tabular} Chem. Name SODIUM CHLORIDE

\section{Fire}

Sudden Release Sudden Releas
of Pressure

Reactivity

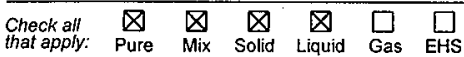
EHS Name

CASL \begin{tabular}{l|l|l|l|l|l|l|}
\hline & & 1 & 3 & 1 & 0 \\
\hline 7 & 3 \\
\hline
\end{tabular} \begin{tabular}{|l|l|}
\hline Trade \\
Secret
\end{tabular} Chem. Name SODIUM HYDROXIDE

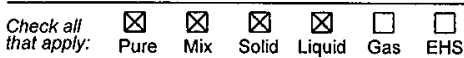
EHS Name \begin{tabular}{|l|l|l|l|l|l|l|l|l|}
\hline CAS & & 1 & 3 & 1 & 0 \\
\hline 7 & 3 \\
\hline
\end{tabular}$\quad \begin{aligned} & \text { Trade } \\
& \text { Secret }\end{aligned}$ Chem. Name SODIUM HYDROXIDE

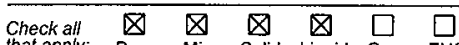
that apply: Pure Mix Solid Liquid Gas EHS EHS Name Certification (Read and sign after completing all sections) I certify under penalty of law that I have personally examined and am familiar with the information submitted in pages one through 70
inquiry of those individuals responsible for obtaining the information, / believe that the submitted information is true, accurate, and complete.

James E. Rasmussen, Director of Environmental Assurance, Permits and Policy Division. Fire of Pressure $\mathrm{X}$ Reactivity $\mathrm{X}$ Immediate (acute) Delayed (chronic)

\begin{tabular}{|c|c|c|c|c|c|}
\hline$\because$ & & truentory & $\begin{array}{l}T \\
\mathbf{p} \\
\mathrm{e}\end{array}$ & $\begin{array}{l}P \\
f \\
s\end{array}$ & $\begin{array}{l}\mathrm{T} \\
\mathrm{p} \\
\mathrm{p}\end{array}$ \\
\hline 0 & 4 & $\begin{array}{l}\text { Max, Dailly } \\
\text { Amount }(c o d \theta)\end{array}$ & B & 1 & 4 \\
\hline & & & $\mathbb{N}$ & 1 & 4 \\
\hline 0 & 4 & Avg Daily & D & 1 & 4 \\
\hline & & & $\mathrm{M}$ & 1 & 4 \\
\hline 3 & 6 & ${ }^{2}$ & $\mathrm{~N}$ & 1 & 4 \\
\hline & & & & 1 & \\
\hline
\end{tabular}
$\square$ Check Check if information below is identical to the information submitted last Storage Codes and Locations (Non-Cónficential) Storage Locations

384 STEAM PLANT, DEACTIVATED $4732 B$ WAREHOUSE 6092I PUMRHOUSE

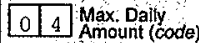
Sudden Release of Pressure $\mathrm{X}$ Reactivity $\mathrm{X}$ Immediate (acute) Delayed (chronic)

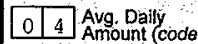
\begin{tabular}{|l|l|l|l|}
\hline 3 & 6 & 5 & No of Days \\
On-Site (days
\end{tabular} \begin{tabular}{|l|l|l|}
\hline 0 & 4 & Maxi Daily \\
\hline Amount & code)
\end{tabular} \begin{tabular}{l|l}
0 & 4 \\
\hline
\end{tabular} \begin{tabular}{|l|l|l|}
\hline 3 & 6 & 5 \\
No. of Days \\
On-Site (days)
\end{tabular} $622 \mathrm{CAB}$ $622 \mathrm{R} \perp \mathrm{AB}$ 6266 LAB

\begin{tabular}{|l|l|l|}
\hline$C$ & 1 & 4 \\
\hline$D$ & 1 & 4 \\
\hline$I$ & 1 & 4 \\
\hline$M$ & 1 & 4 \\
\hline$N$ & 1 & 4 \\
\hline & 1 & 4 \\
\hline
\end{tabular}

1171 SHOP, DEACTIVATED 1171 SHOR, DEACTIVATED 1171 SHOP, DEACTIVATED $1706 \mathrm{KE} \mathrm{IAB}$ $1706 \mathrm{KE}, \mathrm{AB}$ 1714N STORAGE BUILDING

1828 PUMPHOUSE, SECOND ELOOR 100R/C AREA $183 N$ FILTER PLANT 183 N FILTER PLANT RROCESS TANKS OUTSIDE $2025 \mathrm{E}$ 2025E RROCESS TANKS CONEX BOXES E OF $2025 \mathrm{E}$ HAMMER EACILITY NE OF 200W AREA NE OF 200W AREA E OF 200W AREA $1100 \mathrm{ABEA}$ $1100 \quad A R E A$ $100 K$ AREA $100 K$ AREA $100 \mathrm{~N}$ AREA and that based on my coordinate abbreviations

\begin{tabular}{|c|c|c|c|c|}
\hline $\mathrm{N}$ & 1 & 4 & 1828 PUMPHOUSE, SECOND ELOOR & $100 \mathrm{~B} / \mathrm{C}$ AREA \\
\hline E & 1 & 4 & 183N FILTER PLANT & 100N_AREA \\
\hline F & 1 & 4 & 183N EILTER PLANT & 100N AREA \\
\hline A & 1 & 4 & RROCESS TANKS OUTSIDE $2025 \mathrm{E}$ & 200E AREA \\
\hline $\mathrm{C}$ & 1 & 4 & 2025E PROCESS TANKS & 200E AREA \\
\hline E & 1 & 4 & CONEX BOXES E OF $2025 \mathrm{E}$ & $200 E$ AREA \\
\hline
\end{tabular}
$1100 \quad A B E A$ 


\section{TIER TWO}

EMERGENCY AND

HAZARDOUS

CHEMICAL

INVENTORY

$\mid \begin{aligned} & \text { Specific } \\ & \text { information } \\ & \text { by Chemical }\end{aligned}$

Important: Read all instructions before completing form

Chemical Description
WA7 890008967

Owner/Operator Name
Page 54 of 70 pages

Name_U. S. DERARTMENT OF ENERGY Mail Address_P.O. BOX 550, RICHLAND WA 99352

Phone (509) 376-7411.

For
Official
Use
Only

Street 825 JADWIN AVENUE

City _ RICHLAND _ C County BENTON_ State WA Zip 99352

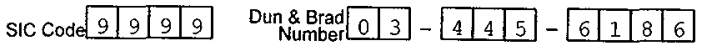

Name PATROL, OPERATIONS CENTER Phone $\{509\} 373-3800$

Name

Phone \begin{tabular}{l|l|l|l|l|l} 
Reporting Period: From January 1 to December 31, 1998 \\
\hline
\end{tabular}
CAS \begin{tabular}{|l|l|l|l|l|l|l|l|}
\hline & 1 & 3 & 1 & 0 \\
\hline 7 & 3 & 2 \\
\hline
\end{tabular}

Chem. Name SODIUM HYDROXIDE

\section{.}

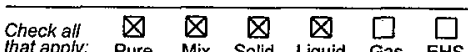

EHS Name

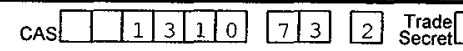

Chem. Name SODIUM HYDROXIDF

\begin{tabular}{|c|c|c|c|c|c|}
\hline $\begin{array}{l}\text { heck all } \\
\text { lat apply: }\end{array}$ & $\underset{\text { Pure }}{\bigotimes}$ & $\underset{\text { Mix }}{\bigotimes}$ & $\underset{\text { Solid }}{\bigotimes}$ & $\underset{\text { Liquid }}{\otimes}$ & $\square$ \\
\hline
\end{tabular}

EHS Name

\begin{tabular}{l|l|l|l|l|l|l|l|l|l|l|l|l|l|}
\hline CAS & & 1 & 3 & 1 & 0 \\
\hline
\end{tabular}

Chem. Name SODIUM HYDBOXIDE

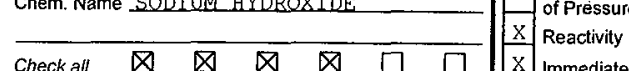

that apply: Pure Mix Solid Liquid Gas EHS

EHS Name

Cẹtification (Read and sign after completing all sections)

\section{Physical and Heards} (Check all that apply)

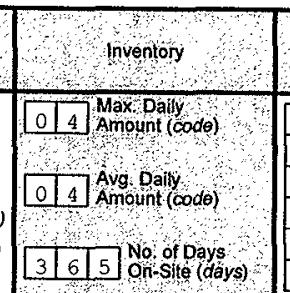

\begin{tabular}{|l|l|l|}
\hline 0 & 4 & Max Daly \\
\hline
\end{tabular}

Sudden Release

$\Rightarrow$ of Pressure

$\mathrm{x}$ Reactivity

$\mathrm{X}$ Immediate (acute)

Delayed (chronic)

\begin{tabular}{|l|l|l|}
\hline 3 & 6 & 5 \\
\hline
\end{tabular}

\begin{tabular}{|l|l|l}
\hline 0 & 4 & Max Daily \\
Amcunt (code)
\end{tabular}

Fire

Sudden Release

$\mathrm{X}$ Immediate (acute)

Delayed (chronic) \begin{tabular}{|l|l|l}
\hline 0 & 4 & Avg Daily \\
\hline
\end{tabular} \begin{tabular}{|lll}
\hline 0 & 4 & Ayg Daily \\
\hline
\end{tabular}

\begin{tabular}{|c|c|c|}
\hline $\begin{array}{l}\mathrm{T} \\
\mathrm{P}\end{array}$ & $\begin{array}{l}\rho \\
\beta \\
\$\end{array}$ & $\begin{array}{l}\mathrm{T} \\
\mathrm{m} \\
\mathrm{p}\end{array}$ \\
\hline 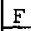 & 1 & 4 \\
\hline$I$ & 1 & 4 \\
\hline$I$ & 1 & 4 \\
\hline$M$ & 1 & 4 \\
\hline$\underline{N}$ & 1 & 4 \\
\hline 0 & 1 & 4 \\
\hline C & 1 & 4 \\
\hline $\mathrm{J}$ & 1 & 4 \\
\hline D & 1 & 4 \\
\hline $\mathrm{E}$ & 1 & 4 \\
\hline A & 1 & 4 \\
\hline I & 1 & 4 \\
\hline I & 1 & 4 \\
\hline $\mathrm{J}$ & 1 & 4 \\
\hline $\mathrm{N}$ & 1 & 6 \\
\hline $\mathrm{N}$ & 1 & 4 \\
\hline$M$ & 1 & 4 \\
\hline $\mathrm{N}$ & 1 & 4 \\
\hline
\end{tabular}

$T$
p
p

\begin{tabular}{|l|l|l|l}
\hline E & 1 & 4 & \\
$2025 E$ & BUILDING, ROOM 112
\end{tabular}

CONEX BOXES E OF $2025 \mathrm{E}$

$2025 \mathrm{E}$ BUILDING ROOM 112

$2025 \mathrm{E}$ BUTLDING, ROOM 134

2025E BUILDING, ROOM $112^{\circ}$

2025E BUILDING, ROOM 134

$204 A R$ BUILDTNG

CONEX BOX BY 204AR

$2101 \mathrm{M}$ BUILDING

2101M WAREHOUSE

TANK BY 211BA

$221 T$ CANYON

STORAGE UNIT SW OF $211 \mathrm{~T}$

$221 T$ CANYON

$222 S$ IAB $4 B$

$222 S$ IAB

$222 \mathrm{SA} \perp A B$

222 SA LAB
Title SHIET COMMANDER

Title

(509) 373-3800

1)

II certify under penalty of law that I have personally examined and am familiar with the information submitted in pages one through .70 iinquiry of those individuals responsible for obtaining the information, I believe that the submitteg iformation is true, accurate, and complete. James E. Rasmussen, Director of Environmental iName and official title of ownerioperator OR owner/operator's authorized representative

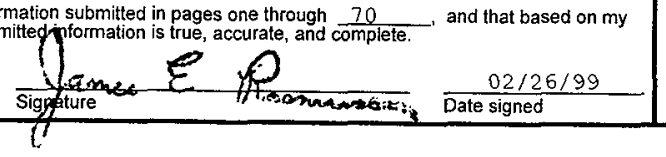
and that based on my

200E AREA $200 E$ AREA $200 E$ AREA 200E AREA 200E AREA $200 \mathrm{E}$ AREA $\square$ 200E AREA 200E AREA 200E AREA $200 E \triangle A B E A$ 2QOE AREA 200W AREA 200W AREA 200W ABEA $200 W$ ABEA 200W AREA 200W AREA 200W AREA ptional Attachments

$x$ I have attached a site plan I have attached a list of site coordinate abbreviations I have attached a description of dikes and other safeguard measures A-6000-633 (02198) 
Washington Community Right-To-Know \# :

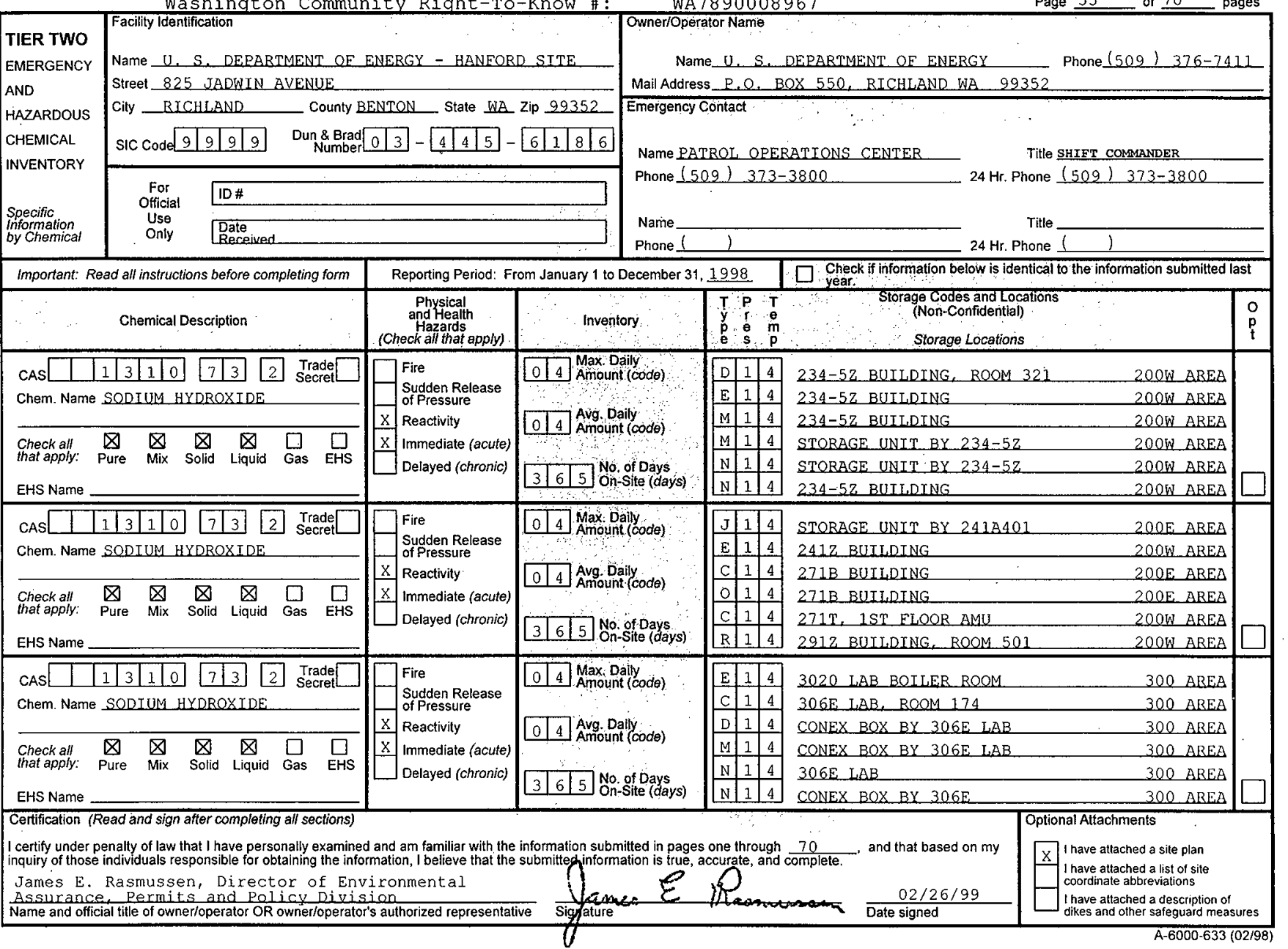


Washington Community Right-To-know \#:

\begin{tabular}{|c|c|}
\hline $\begin{array}{l}\text { TIER TWO } \\
\text { EMERGENCY } \\
\text { AND } \\
\text { HAZARDOUS }\end{array}$ & $\begin{array}{l}\text { Facility Identification } \\
\text { Name U. S. DERARTMENT OF ENERGY - HANEORD SITE } \\
\text { Street } 825 \text { JADWIN AVENUE } \\
\text { City_RICHLAND County EENTON State WA Zip } 99352\end{array}$ \\
\hline CHEMICAL & 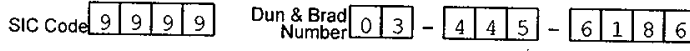 \\
\hline $\begin{array}{l}\text { Specific } \\
\text { Information } \\
\text { by Chemical }\end{array}$ & $\begin{array}{l}\text { ID \# } \\
\text { Oate } \\
\text { Received }\end{array}$ \\
\hline
\end{tabular}

Name U. S. DEPARTMENT OF ENERGY Phone (509) $376-7911$ Mail Address D. O. BOX 550 RICHLAND WA 99352 Emergency Contact

Name PATROL OPERATIONS CENTER Phone (509) 373-3800 Name

Phone (1) Reporting Period: From January 1 to December 31, 1998 Physical and Health (Check allthat oppiy) Chemical Description

\section{Fire}

Trade

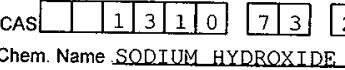

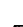

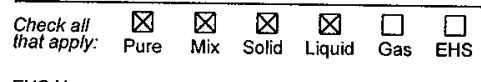
EHS Name CAS \begin{tabular}{|l|l|l|l|l|l|l|l|}
\hline & & 1 & 3 & 1 & 0 \\
\hline
\end{tabular} Chem. Name SODYUM HYDROXIDE

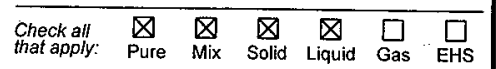
EHS Name

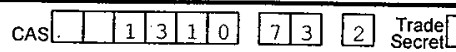
Chem. Name SODIUM BYDROXIDE

\begin{tabular}{|c|c|c|c|c|c|c|}
\hline $\begin{array}{l}\text { Check all } \\
\text { that apply: }\end{array}$ & $\underset{\text { Pure }}{\bigotimes}$ & $\underset{\text { Mix }}{\bigotimes}$ & $\underset{\text { Solid }}{\bigotimes}$ & $\underset{\text { Liquid }}{\otimes}$ & $\square$ & $\square$ \\
\hline
\end{tabular}

EHS Name Centification (Read and sign after completing all sections) Sudden Release of Pressure $X$ Reactivity $x$ immediate (acule) Delayed (chronic) Fire \begin{tabular}{|l|l|}
\hline & $\begin{array}{l}\text { Sudden Release } \\
\text { of Pressure } \\
\mathrm{X}\end{array}$ \\
$\mathrm{X}$ & $\begin{array}{l}\text { Reactivity } \\
\text { Immediate (acute) } \\
\text { Delayed (chronic) }\end{array}$ \\
\hline
\end{tabular}

\begin{tabular}{|l} 
Fire \\
$\begin{array}{l}\text { Sudden Release } \\
\text { of Pressure }\end{array}$
\end{tabular}

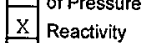
$\mathrm{X}$ immediate (acute) Delayed (chronic)

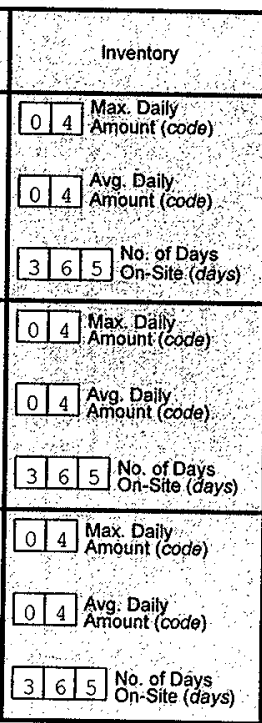

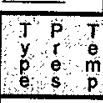

\begin{tabular}{|l|l|l|}
\hline$A$ & 1 & 4 \\
\hline$N$ & 1 & 4 \\
\hline$N$ & 1 & 4 \\
\hline$D$ & 1 & 4 \\
\hline$F$ & 1 & 4 \\
\hline$N$ & 1 & 4 \\
\hline
\end{tabular}

Storage Codes and Location
(Non-Confidential)
storage Locations

Title SHIET COMMANDER $24 \mathrm{Hr}$. Phone (509) 373-3800

Title $24 \mathrm{Hr}$. Phone 1 Check if information betow is identical to the information submilted last year.

\begin{tabular}{|c|c|}
\hline DANK E OF 310 FACILITY & 300 AREA \\
\hline $327 \mathrm{LAB}$ & $300 \quad A B E A$ \\
\hline 331 BUILDING & $300 \_A R E A$ \\
\hline 331D BUILDING & 300 AREA \\
\hline 3310 BUILDING & 300 AREA \\
\hline $331 D$ BUILDING & 300 AREA \\
\hline 3705 PHOTOGRAPHY LAB. & 300 AREA \\
\hline 3705 PHOTOGRAPHY LAB & 300 AREA \\
\hline 382 PUMP HOUSE & 300 AREA \\
\hline 4732B WAREHOUSE & 400 AREA \\
\hline 6092I PUMRHOUSE & HAMMER FACILITY \\
\hline $622 \mathrm{R} \perp \mathrm{LAB}$ & NE OE $200 W$ AREA \\
\hline $6266 \mathrm{LAB}$ & $E$ OF $200 \mathrm{~W}$ AREA \\
\hline $6266 \mathrm{LAB}$ & $E$ OF $200 W$ AREA \\
\hline 6268 CLEANING STATION & $E$ OE $200 W$ AREA \\
\hline 6269 MOBILE LAB STORAGE & E OE 200W AREA \\
\hline
\end{tabular}

\begin{tabular}{|l|l|l|}
\hline$M$ & 1 & 4 \\
\hline & 1 & \\
\hline
\end{tabular}
\begin{tabular}{|l|l|l|}
\hline $\mathrm{N}$ & $\mathrm{I}$ & 4 \\
\hline
\end{tabular}

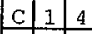
\begin{tabular}{|l|l|l|}
\hline$N$ & 1 & 4 \\
\hline$D$ & 1 & 4 \\
\hline
\end{tabular} \begin{tabular}{l|l|l|}
\hline$D$ & 1 & 4 \\
\hline & & 1 \\
\hline
\end{tabular} \begin{tabular}{|l|l|l|}
\hline $\mathrm{N}$ & 1 & 4 \\
\hline
\end{tabular}

\begin{tabular}{|l|l|l|}
\hline$M$ & 1 & 4 \\
\hline$N$ & 1 & 4 \\
\hline$N$ & 1 & 4 \\
\hline$N$ & 1 & 4 \\
\hline & & \\
\hline & & \\
\hline
\end{tabular}

$02 / 26 / 99$ Date signed

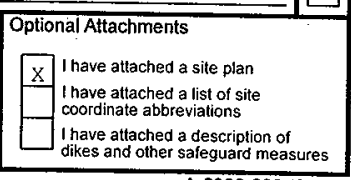

A-6000-633(02/98) 


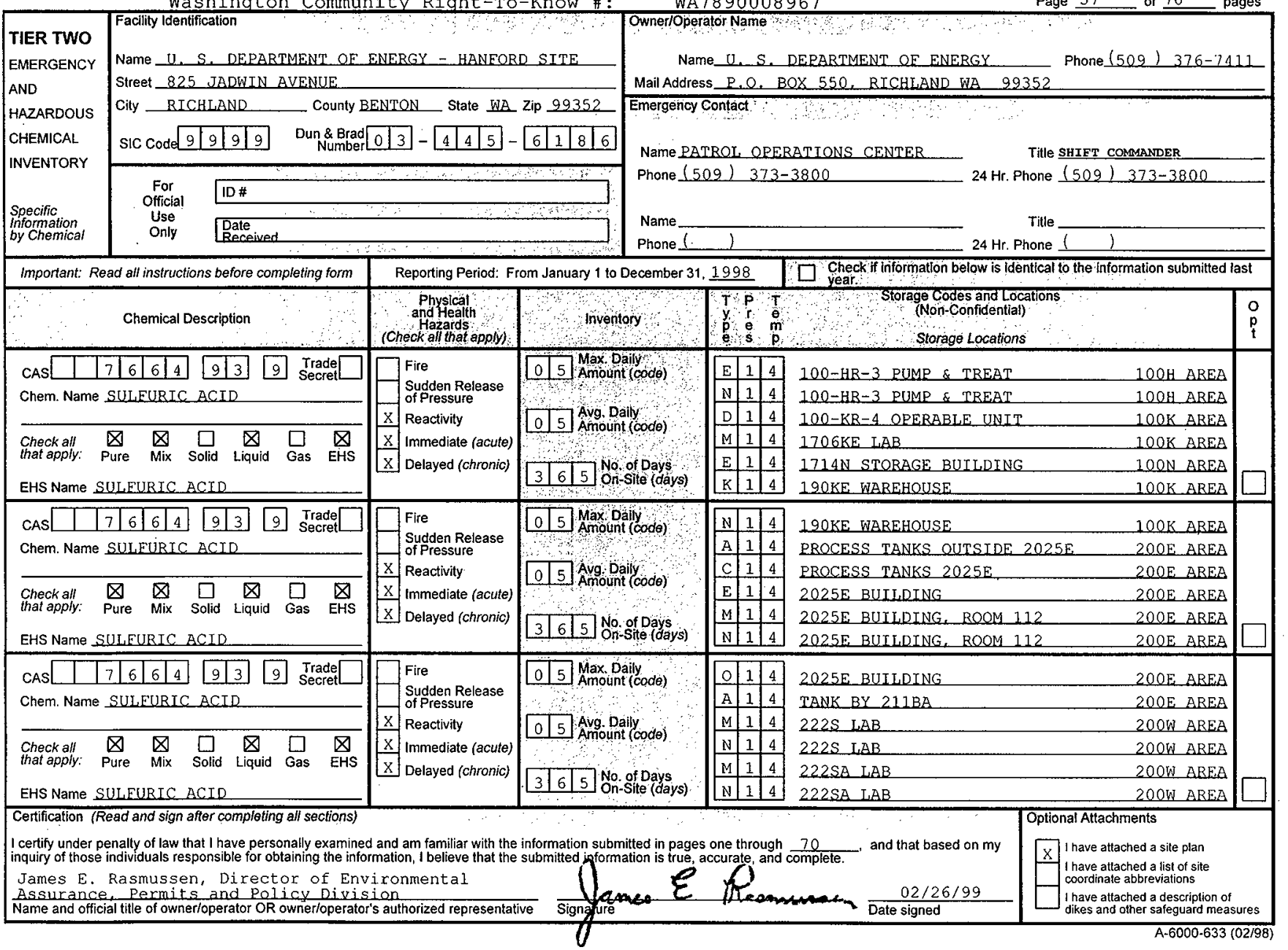




\section{TIER TWO}

EMERGENCY

AND

HAZARDOUS

CHEMICAL

INVENTORY

Specific

Intormation
by Chemical

Name U. S. DEPARTMENT OF ENERGY - HANFORD SITF

Street 825 JADWIN AVENUE:

City _ BICHLAND _ County BENTON_ State WA Zip 99352

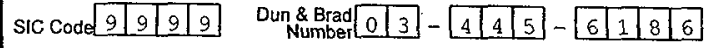

For $10 \#$
Official
Use
Only Daty
Beceived
Owner/Operatôn Name

Name_U. S. DEPARTMENT OF ENERGY Mail Address P. O. BOX 550, RICHLAND WA 99352

Phone (509) 376-7411 Emergency Contact

Name PATROL OPERATIONS CENTER

Title SHIFT COMAADER Phone (509) 373-3800 24 Hir. Phone $(509) \quad 373-3800$

Name _____________te

Phone 1 _ $24 \mathrm{Hr}$. Phone $(1$

Important: Read all insfructions before completing form

Chemical Description

Reporting Period: From January 1 to December 31, 1998

Physical
and Health Hazards (Check all that apply)

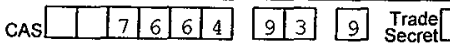

Fire
Sudden Release

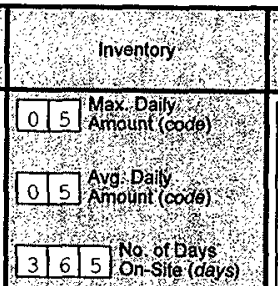

\begin{tabular}{|c|c|c|}
\hline$\frac{f}{y}$ & $\mathrm{P}$ & $\begin{array}{l}\mathrm{T} \\
\mathrm{m}\end{array}$ \\
\hline$M$ & 1 & 4 \\
\hline $\mathrm{N}$ & 1 & 4 \\
\hline $\mathrm{N}$ & 1 & 4 \\
\hline $\mathrm{N}$ & 1 & 4 \\
\hline$M$ & 1 & 4 \\
\hline $\mathrm{N}$ & 1 & \\
\hline
\end{tabular}
Ctieck
Chem. Name SULFURIC ACID

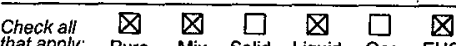

Sudden Rele
of Pressure

$\mathrm{X}$ Reactivity

$\mathrm{X}$ Immediate (acute)

that apply: Pure Mix Solid Liquid Gas EHS

$x$ Delayed (chronic)

EHS Name SULFURIC ACID

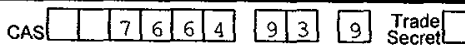
Chem. Name SULFURIC ACID

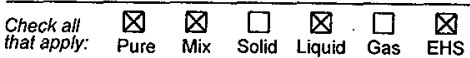
EHS Name SULFURIC ACID

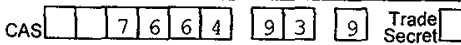
Chem. Name SULFURIC ACID

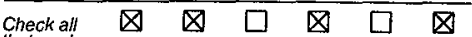
that apply: Pure Mix Solid Liquid Gas EHS

EHS Name SULFURIC ACID

\begin{tabular}{|c|c|}
\hline & Fire \\
\hline & $\begin{array}{l}\text { Sudden Release } \\
\text { of Pressure }\end{array}$ \\
\hline$\underline{x}$ & Reactivity \\
\hline $\mathrm{x}$ & Immediate (acute) \\
\hline$x$ & Delayed (chronic) \\
\hline
\end{tabular}

(1)

DTWax Dally ow

\begin{tabular}{lll}
0 & 5 & Max Dally and \\
\hline
\end{tabular}

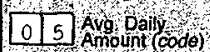

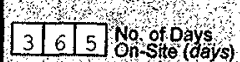

\section{Fire}

Sudden Release of Pressure

$X$ Reactivity $\mathrm{X}$ Immediate (acute) $\mathrm{X}$ Delayed (chronic)

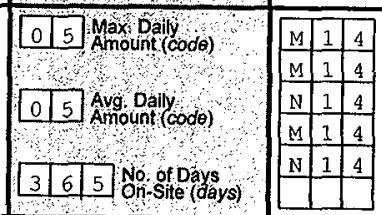

\begin{tabular}{|l|l|l|l|l|l}
\hline$A$ & 1 & 4 \\
\hline
\end{tabular}

$\begin{array}{lll}N & 1 & 4 \\ M & 315 \text { EIITER PLANT }\end{array}$

$\begin{array}{llll}M & 1 & 4 & 3705 \text { PHOTOGRAPHY LAB }\end{array}$

\begin{tabular}{l|l|l|l|l|l}
\hline $\mathrm{N}$ & 1 & 4 \\
\hline
\end{tabular}

CONEX BOX AT PIT 6

6092I PUMPHOUSE

W OE 300 AREA HAMMER FACIIITY

$622 \mathrm{R} \perp \mathrm{LAB}$

$6266 \mathrm{LAB}$

$\mathrm{JE}$ OE 200W AREA

$6266 \mathrm{IAB}$ $E$ OF 200W AREA

6269 MOBIIE LAB STORAGE $E$ OF 200W AREA 6269 MOBILE I.AB STORAGE

AREA 200W AREA 200W AREA 300 AREA 300 AREA $\square$ 300 AREA 300 AREA 300 AREA Certification (Read and sign after completing all sections

II certify under penalty of law that $t$ have personally examined and am familiar with the information submitted in pages one through 70 inquiry of those individuals responsible for obtaining the information, I believe that the submitted in rmation is tru

James E. Rasmussen, Director of Environmental Assurance. Permits and Policy Division Name and official title of owner/operator OR owner/operator's authorized representative
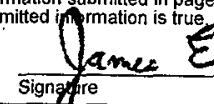

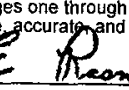

-

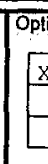

Optional Attachments

$\mathrm{X}$ I have attached a site plan I have attached a list of site coordinate abbreviations dikes and other safeguard measures I have attached a description of 
Washington Community Right-To-know \#:

\section{TIER TWO}

EMERGENCY

AND

HAZARDOUS

CHEMICAL

INVENTORY

Specific

Information

by Chemical
Name $U, S$, DEPARTMENT OF ENERGY - HANEORD SITE

Street 825 JADWIN AVENUE

City _RICHLBND

County BENTON_ State WA Zip 99352

For
Official
Use
Only

SIC Codet

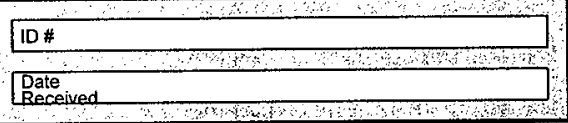

Name_U. S. DERARTMENT OF ENERGY Phone $(509) \quad 376-7411$
Chemical Description
Important: Read all instructions before completing form

Reporting Period: From January 1 to December 31, 1998 and Health Hazaids (Check all that opply)

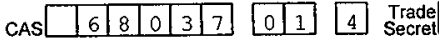

Chem. Name SYNTHETIC PARAEEINIC HYDROCARBON

$\begin{array}{lllll}\text { Check all } & \square & \square & \square & \square \\ \text { that apply: } & \square & \square & \square \\ \text { Pure }\end{array}$ EHS Name

CAS \begin{tabular}{|l|l|l|l|l|l|l|l|l|}
\hline 6 & & & & 7 & 5 & 9 \\
\hline
\end{tabular} Chem. Name IRICHIOROFLUOROMETHANE

$$
\text { (EREON 11) }
$$

$\begin{array}{llllll}\text { Check all } & \bigotimes & \square & \square & \square & \square\end{array}$ that apply: Pure Mix Solid Liquid Gas EHS EHS Name \begin{tabular}{|l|l|l|l|l|l|l|l|l|}
\hline CAS & & & & 7 & 5 & 6 & 9 \\
\hline
\end{tabular} Chem. Name TRICHLOROFLUOROMETHANE. (FREON 11 ).

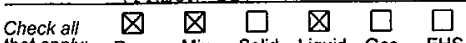
that apply: Pure Mix Solid Liquid Gas EHS

EHS Name

\begin{tabular}{|l|l}
\hline & $\begin{array}{l}\text { Fire } \\
\text { Sudden Release } \\
\text { of Pressure } \\
\text { Reactivity } \\
\mathrm{X}\end{array}$ \\
$\mathrm{y}$ & $\begin{array}{l}\text { Immediate (acute) } \\
\text { Delayed (chronic) }\end{array}$ \\
\end{tabular}

\section{Fire}

$\mathrm{x}$ Sudden Release $\mathrm{X}$ of Pressure

Reactivity

$\mathrm{x}$ Immediate (acute) $\mathrm{X}$ Delayed (chronic)

\begin{tabular}{|c|c|}
\hline & Fire \\
\hline$X$ & $\begin{array}{l}\text { Sudden Release } \\
\text { of Pressure }\end{array}$ \\
\hline & Reactivity \\
\hline $\mathrm{X}$ & Immediate (acute) \\
\hline $\mathrm{x}$ & Delayed (chronic) \\
\hline
\end{tabular}

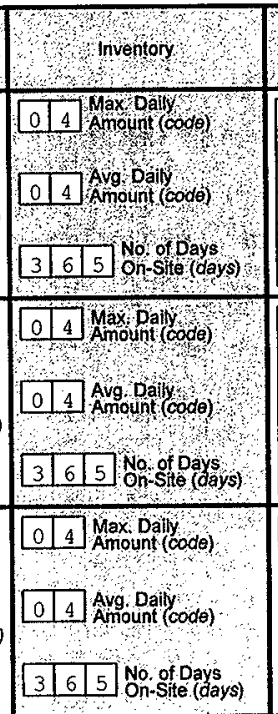

Title SHIFT COMMANDER

Name PATROI, OPERATIONS CENTER Phone $(509) \quad 373-3800$

Name

Phone (1_ )
(509) 373-3800 $24 \mathrm{Hr}$. Phone

\section{Certification (Read and sign after completing all sections)}

I certify under penalty of law that I have personally examined and am familiar with the information inquiry of those individuals responsible for obtaining the information, I believe that the submitted

James E. Rasmussen, Director of Environmental

Assurance, Permits and Policy Division

Name and official title of owner/operator OR owner/operator's authorized representative

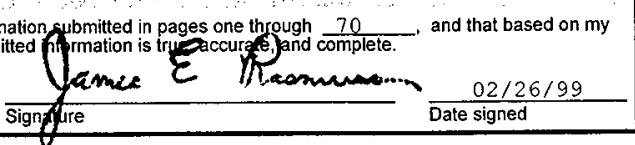
Date signed Creck ff information below is identical to the information submitted last

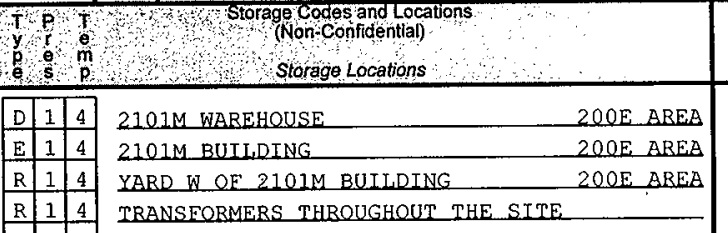

\begin{tabular}{|l|l|l|}
\hline F & 1 & 4 \\
\hline$L$ & 2 & 4 \\
\hline$L$ & 2 & 4 \\
\hline F & 1 & 4 \\
\hline$D$ & 1 & 4 \\
\hline$R$ & 2 & 4 \\
\hline
\end{tabular}

$105 \mathrm{NB}$ SHOP

$100 N$ AREA

1330N WASTE STORAGE $100 N$ AREA

STORAGE BUILDING W OF $272 \mathrm{~S}$ $200 W$ AREA 328 BUILDING, CYIINDER DOCK _. 300 AREA

427 BUII,DING, ROOM 234 400 AREA 427 BUILDING, ROOM 352 400 ABEA

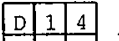

400 AREA

4831 STORAGE BUILDING 607 BATCH PLANT RT 3 BETWEEN 200E/W AREAS

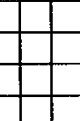




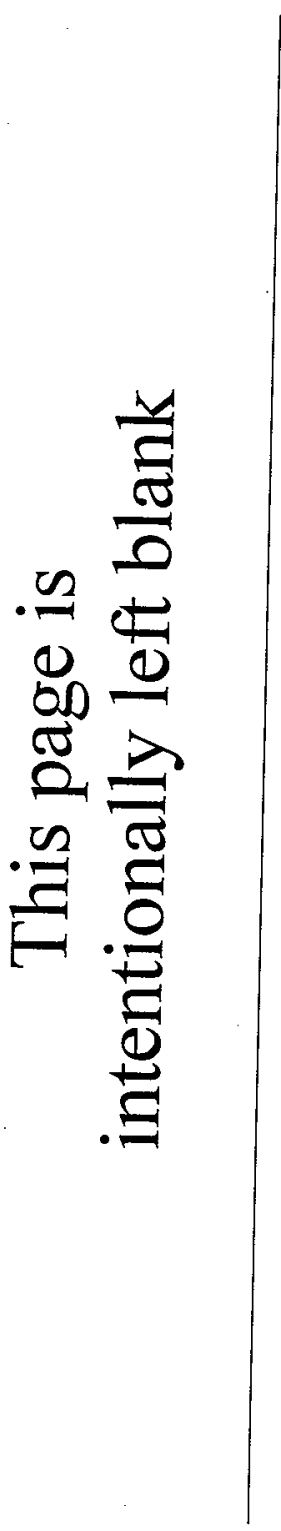




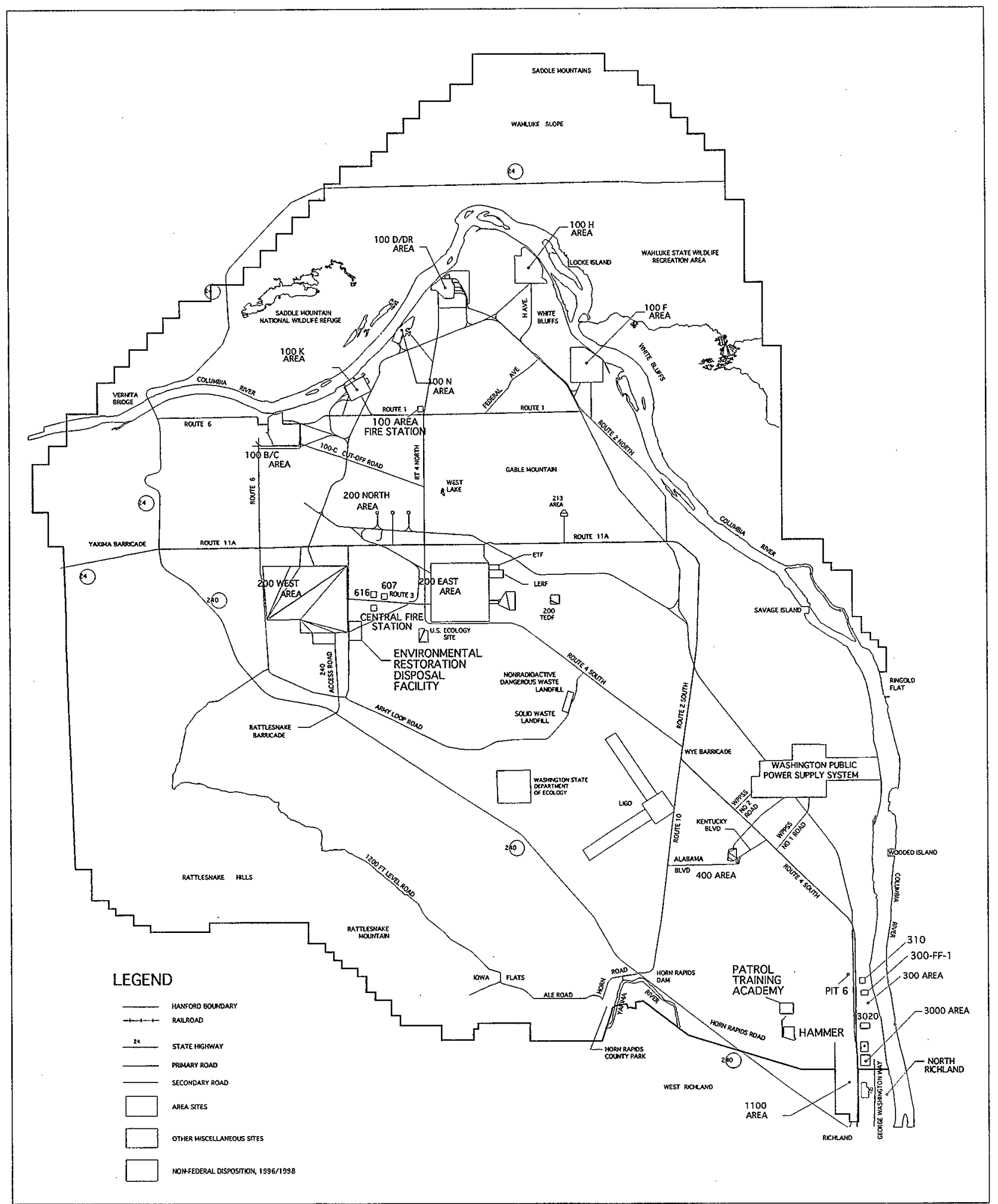

HANFORD SITE

99020104.14

Page 60 of 70 pages 


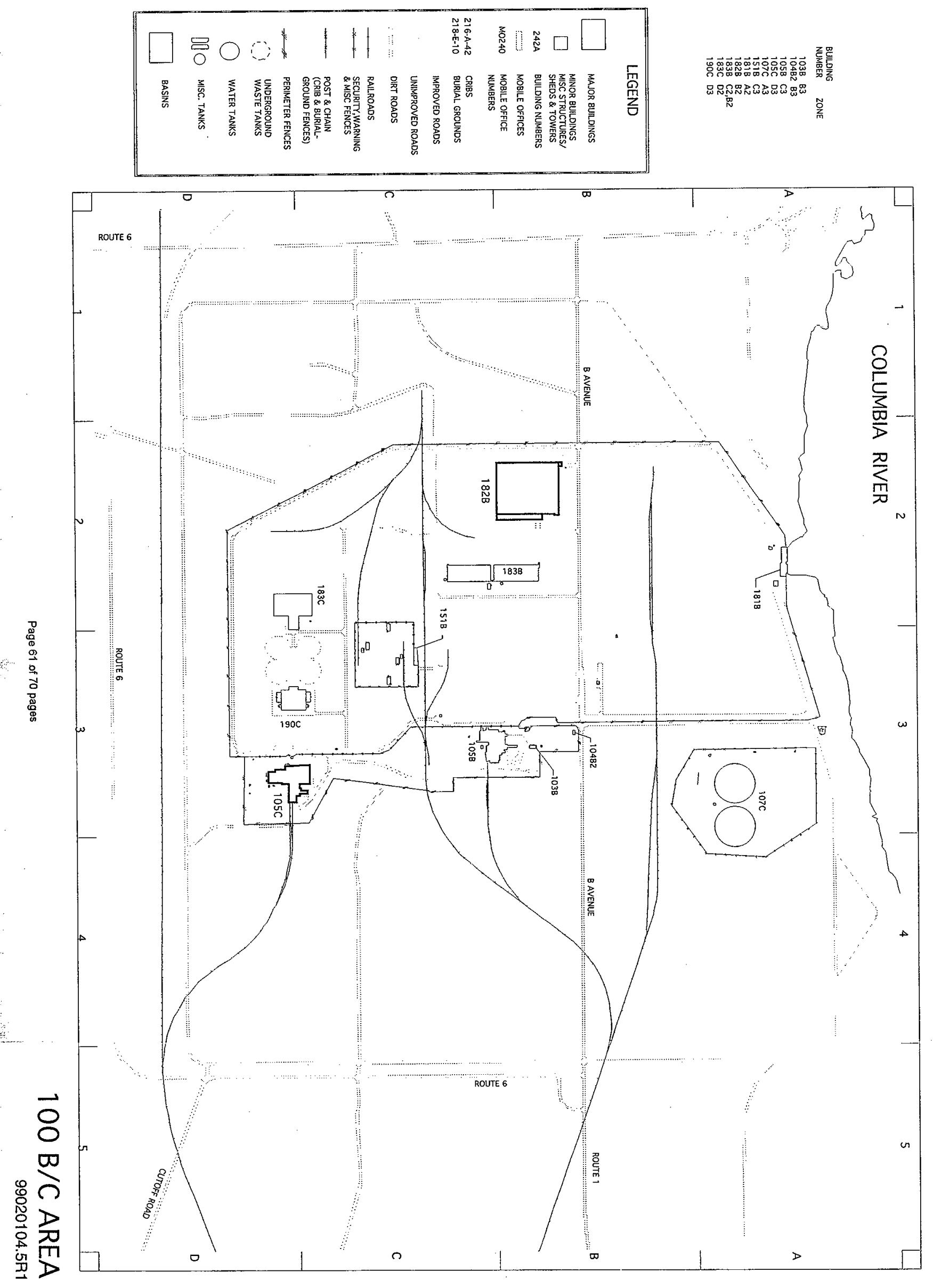




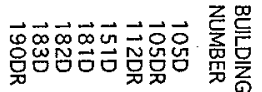

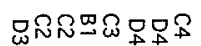

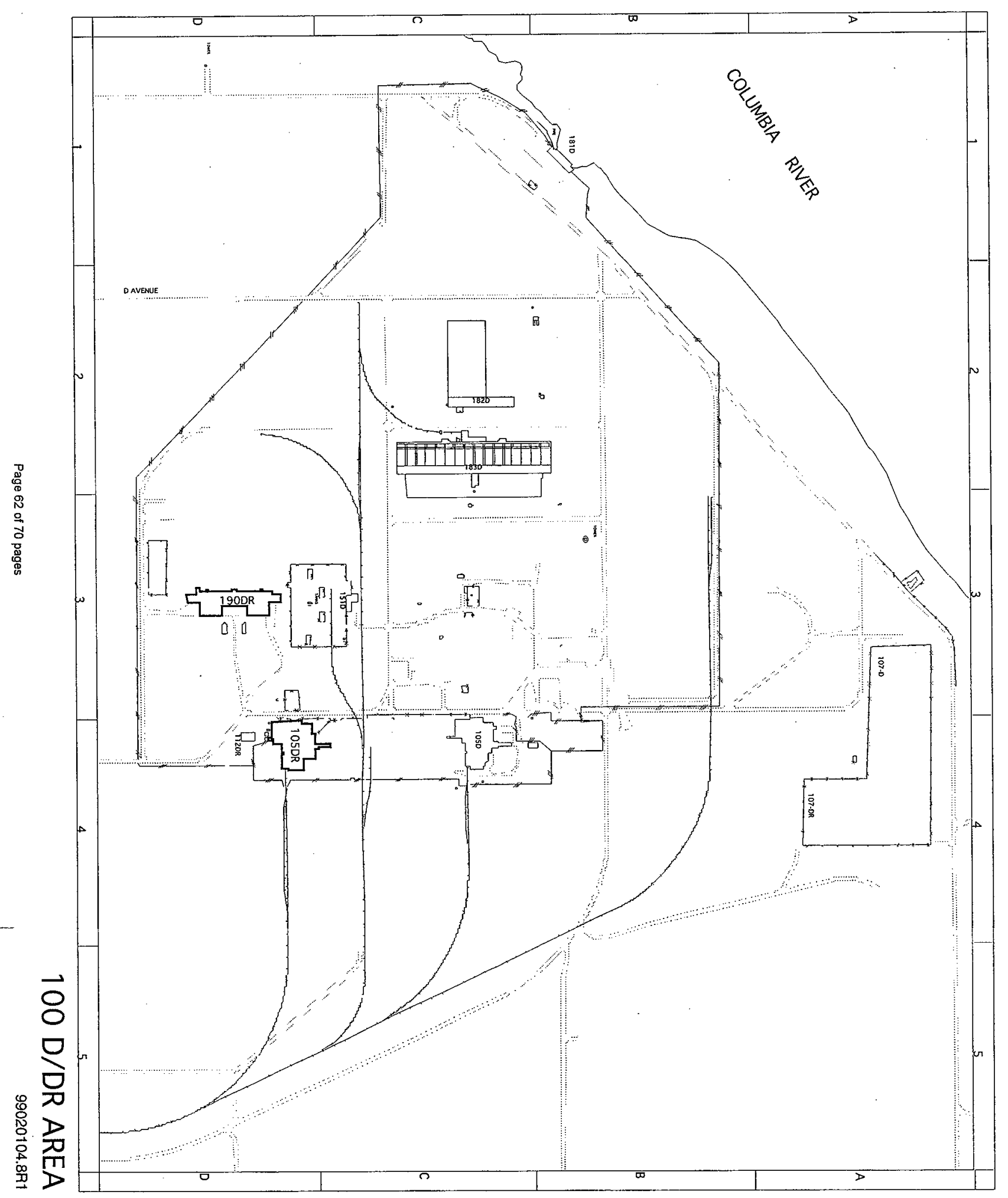




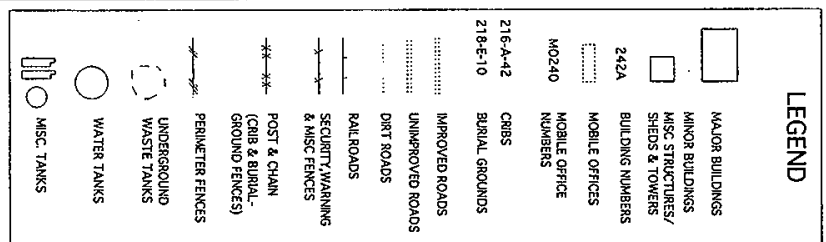

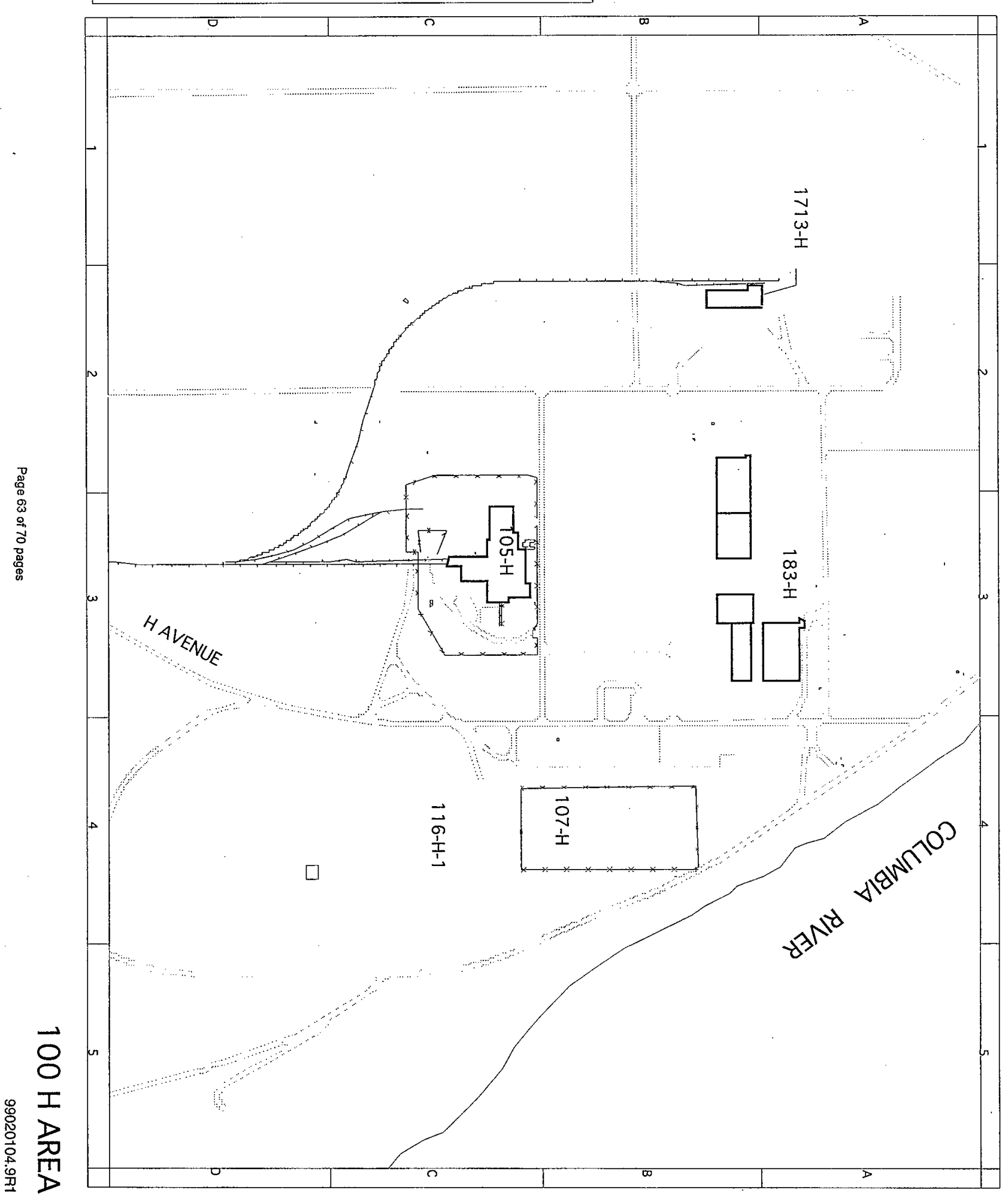



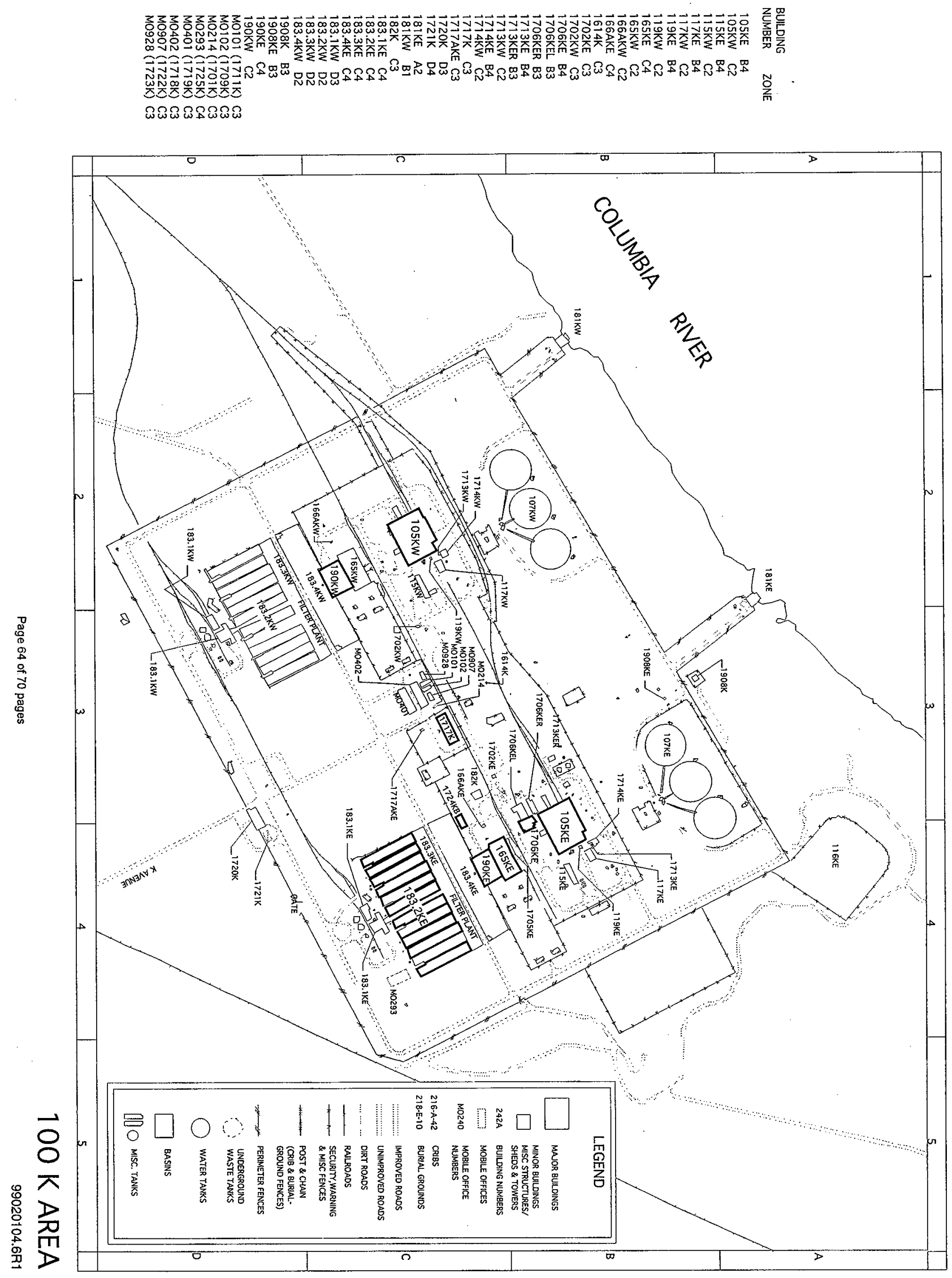


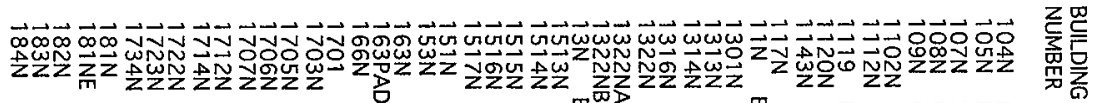

నกన

实

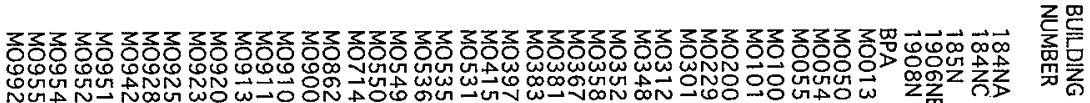

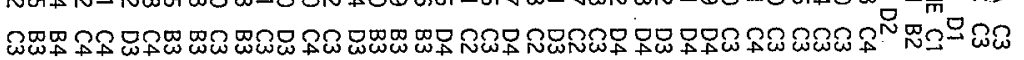

鲕

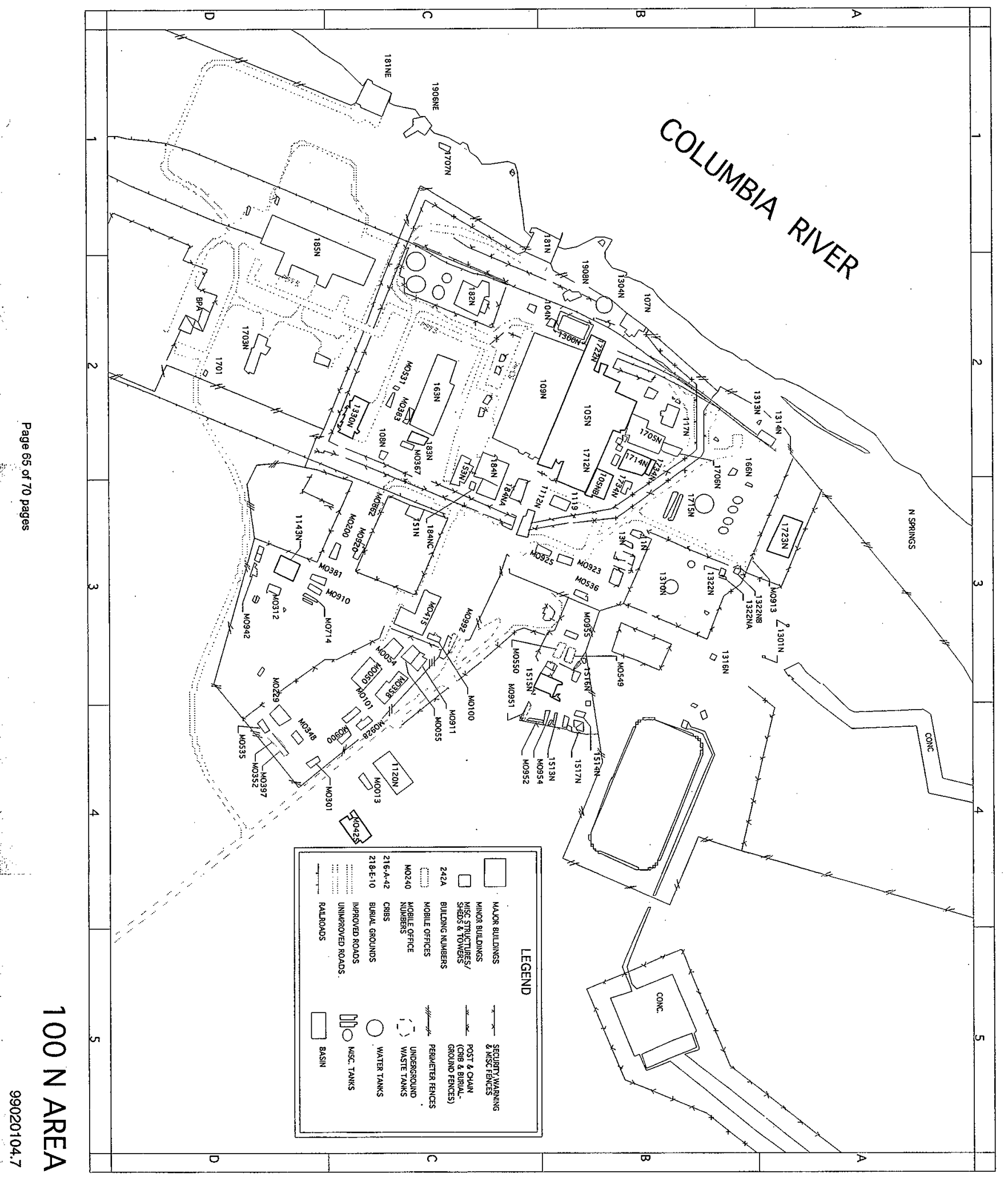




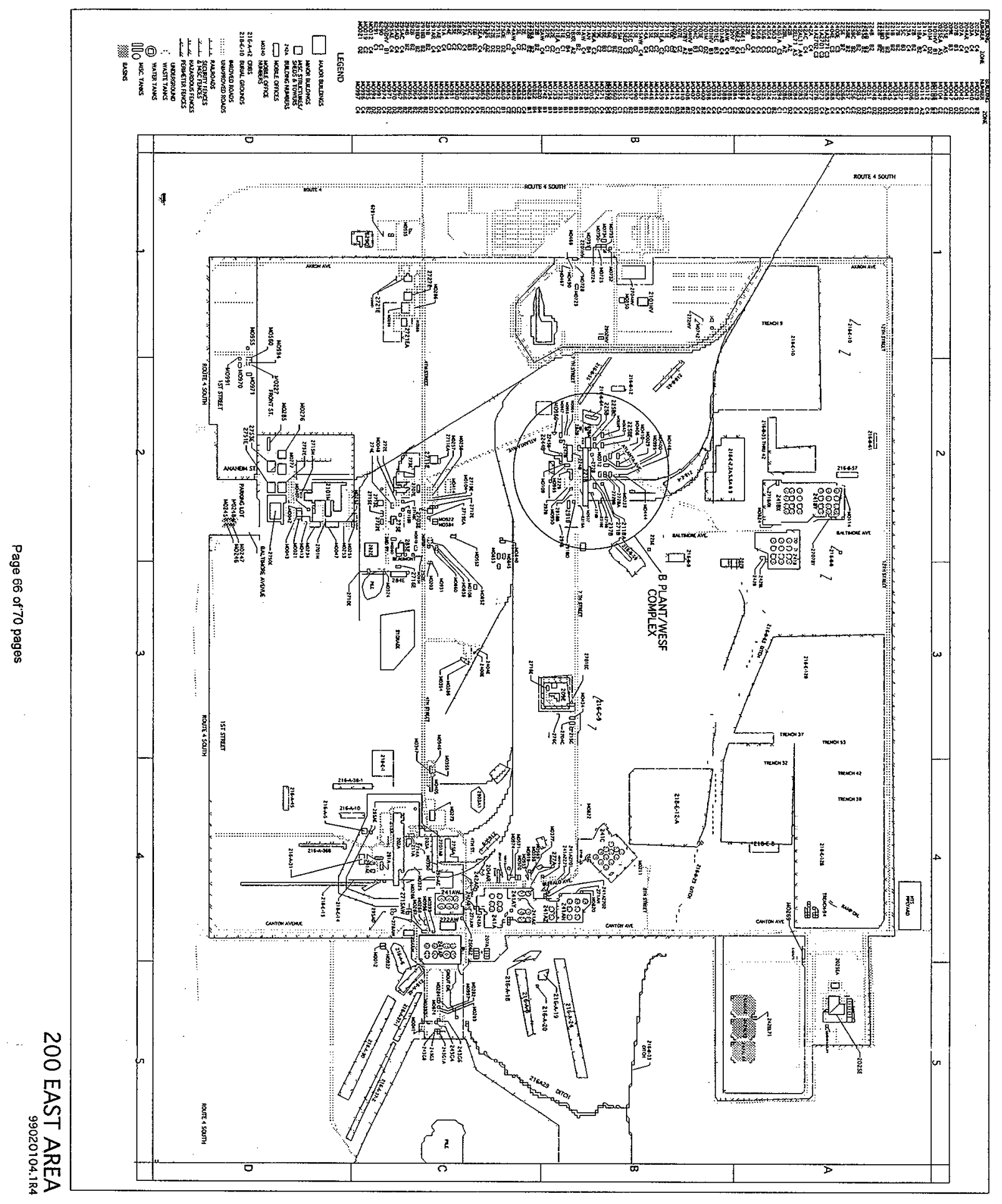




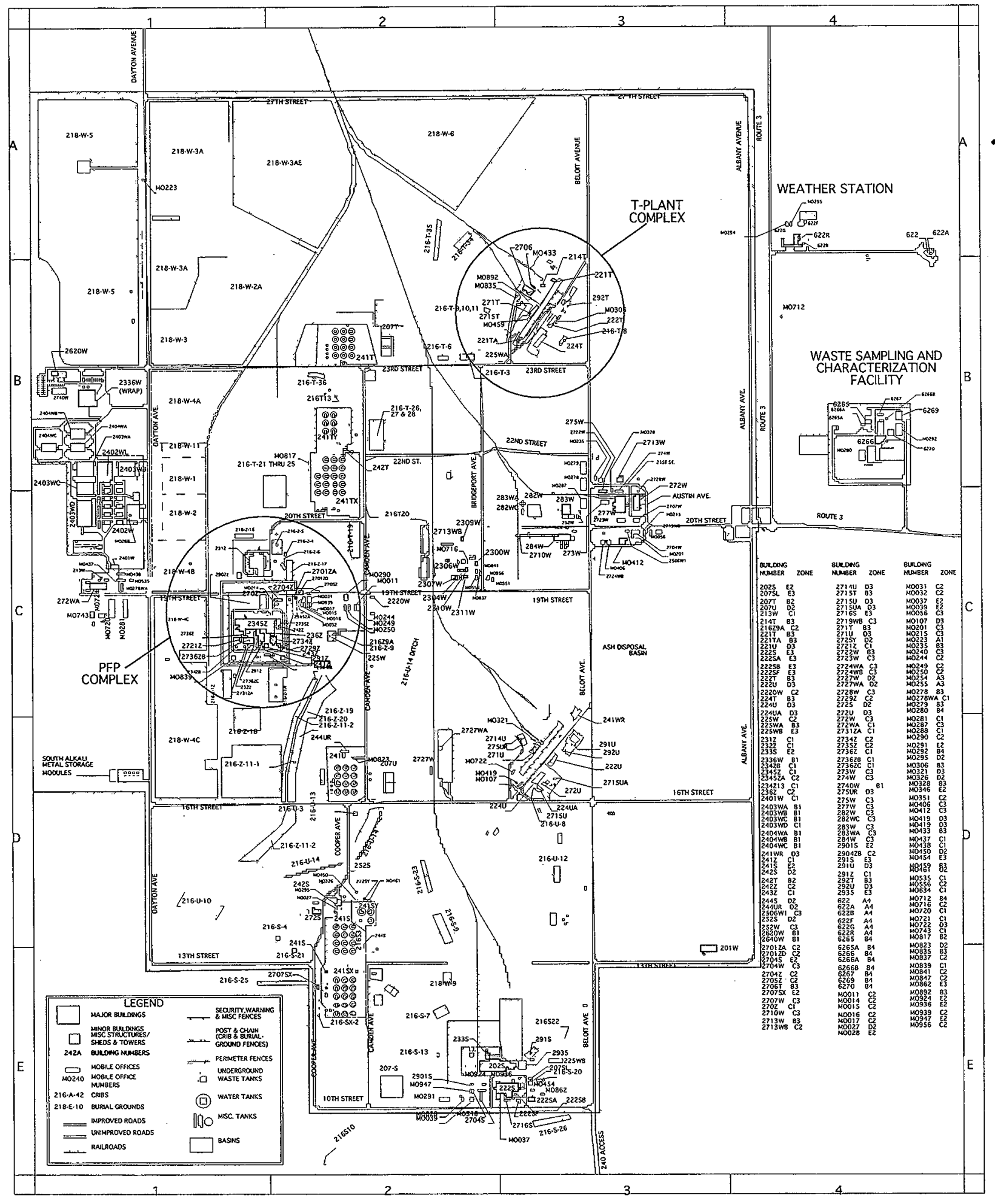

99020104.2 


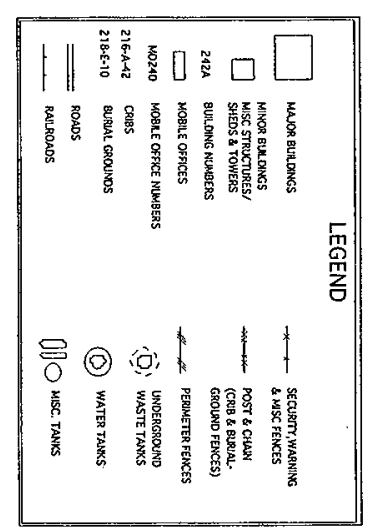

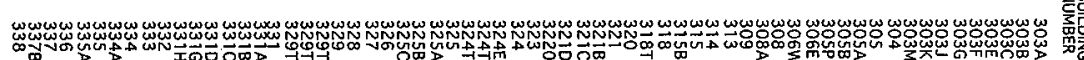

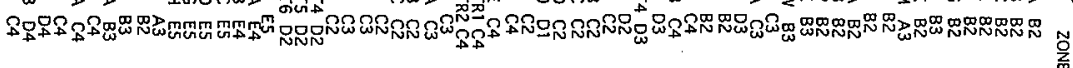

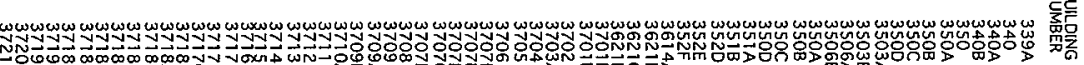

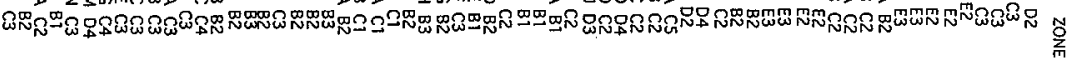

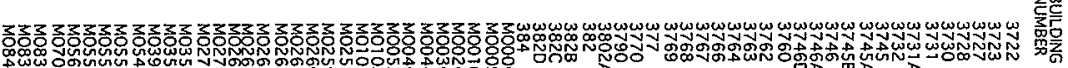

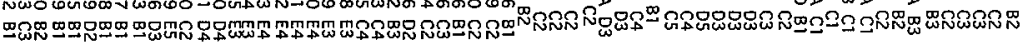

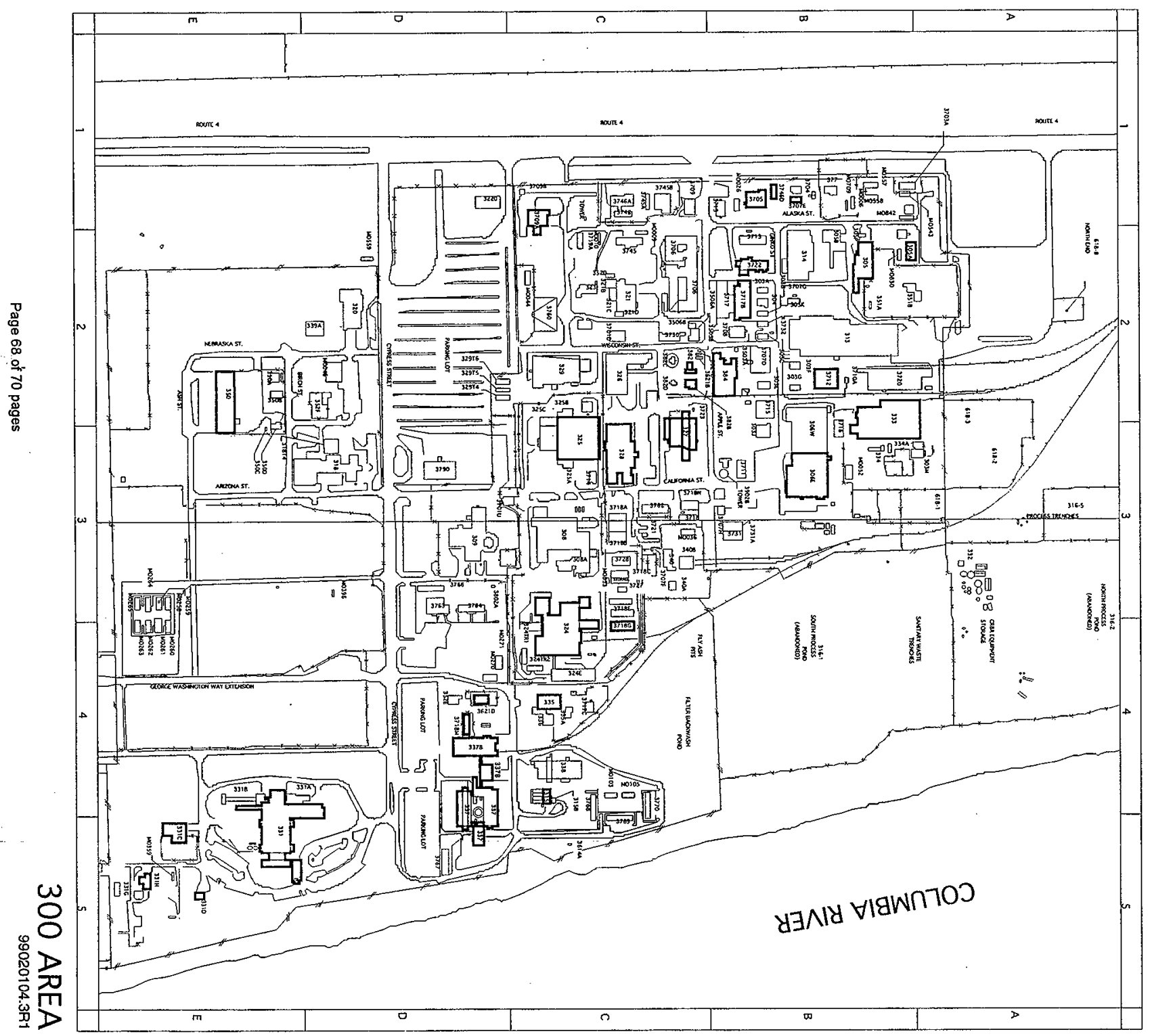




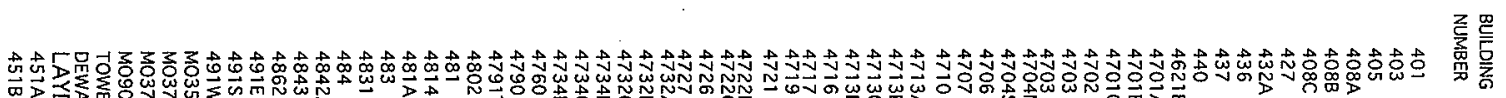

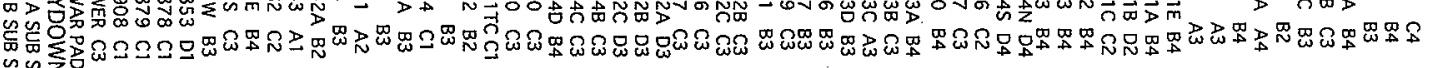

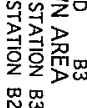

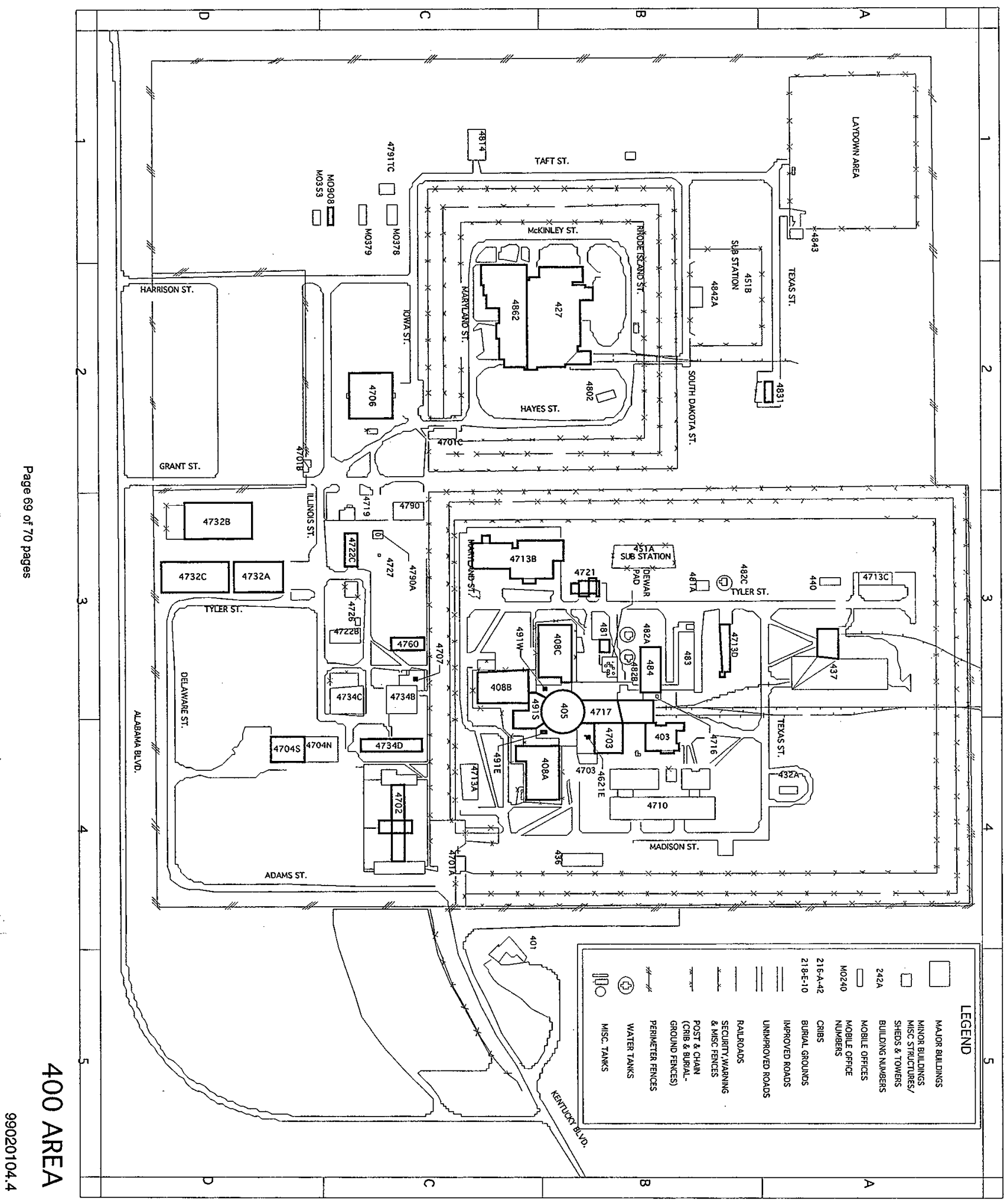




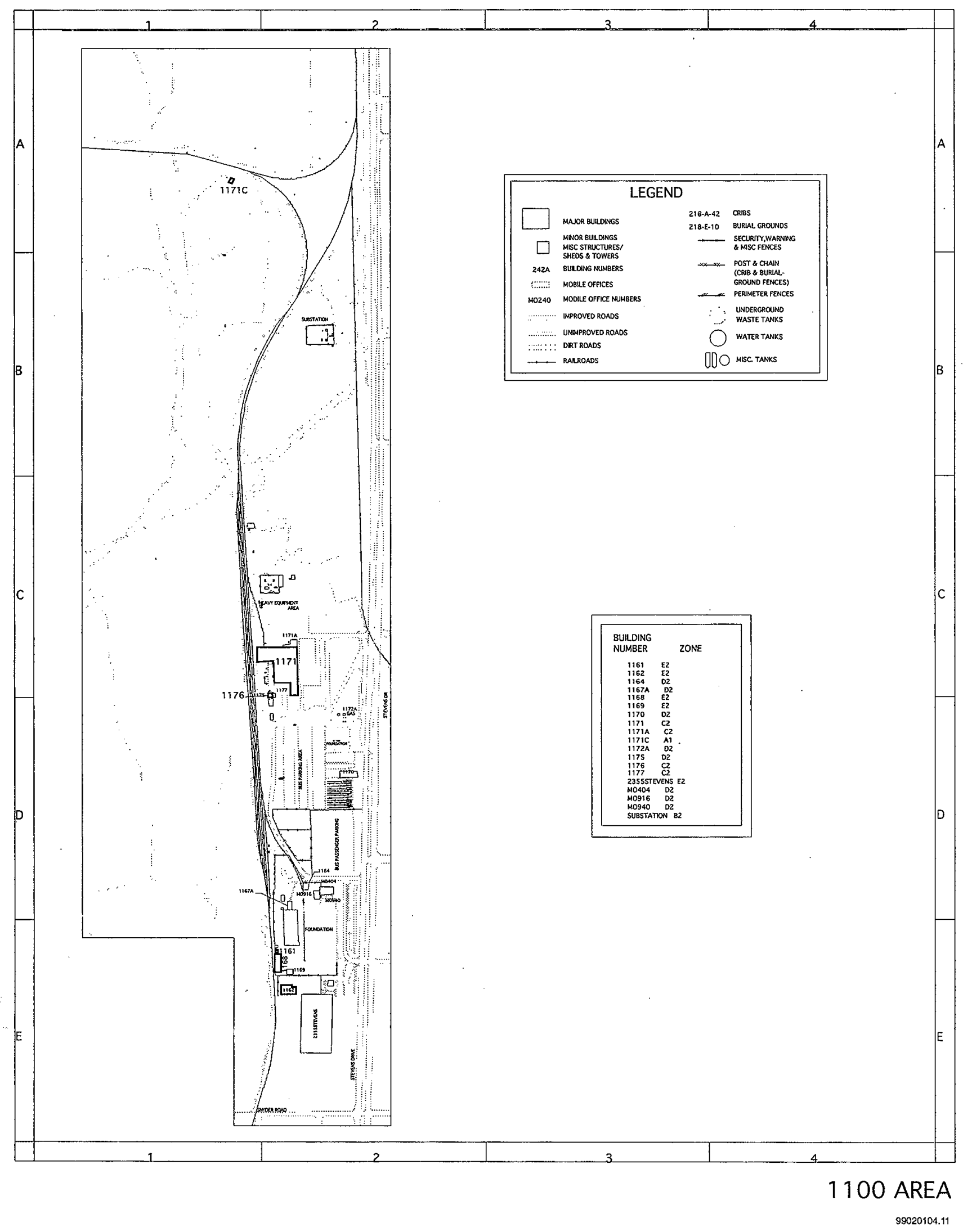

Page 70 of 70 pages 


\section{TIER TWO INSTRUCTIONS}

\section{General Information}

Submission of this Tier Two form (when requested) is required by Title III of the Superfund Amendments and Reauthorization Act of 1986, Section 312, Public Law 99-499, codified at 42 U.S.C. Section 11022. The purpose of the Tier Two form is to provide State and local officials and the public with specific information on hazardous chemicals present at your facility during the past year.

\section{Certification}

The owner or operator or the officially designated representative of the owner or operator must certify that all information included in the Tier Two submission is true, accurate, and complete. On the first page of the Tier Two report, enter your full name and official title. Sign your name and enter the current date. Also, enter the total number of pages included in the Confidential and Non-Confidential Information Sheets as well as all attachments. An original signature is required on at least the first page of the submission. Submissions to the SERC, LEPC, and local fire departments must each contain an original signature on at least the first page. Subsequent pages must contain either an original signature, a photocopy of the original signature, or a signature stamp. Each page must contain the date on which the original signature was affixed to the first page of the submission and the total number of pages in the submission.

\section{Who Must Submit This Form}

Section 312 of Title III requires that the owner or operator of a facility submit this Tier Two form if so requested by a State emergency response commission, a local emergency planning committee, or the local fire departments with jurisdiction over the facility.

This request may apply to the owner or operator of any facility that is required, under regulations implementing the Occupational Safety and Health Act of 1970 , to prepare or have available a Material Safety Data Sheet (MSDS) for a hazardous chemical present at the facility. MSDS requirements are specified in the Occupational Safety and Health Administration (OSHA) Hazard Communication Standard, found in Title 29 of the Code of Federal Regulations at $\$ 1910.1200$. This form does not have to be submitted if all of the chemicals located at your facility are excluded under Section 311(e) of Title III.

\section{What Chemicals Are Included}

If you are submitting Tier Two forms in lieu of Tier One, you must report the required information on this Tier Two form for each hazardous chemical present at your facility in quantities equal to or greater than established threshold amounts (discussed below), unless the chemicals are excluded under Section 3 II (e) of Title III. Hazardous chemicals are any substance for which your facility must maintain an MSDS under OSHA's Hazard Communication Standard. 


\section{Hanford Site Tier Two \\ Emergency and Hazardous Chemical Inventory}

\section{What Chemicals Are Excluded}

Section $311(\mathrm{e})$ of Title III excludes the following substances:

(i) Any food, food additive, color additive, dnug, or cosmetic regulated by the Food and Drug Administration;

(ii) Any substance present as a solid in any manufactured item to the extent exposure to the substance does not occur under normal conditions of use;

(iii) Any substance to the extent it is used for personal, family, or household purposes, or is present in the same form and concentration as a product packaged for distribution and use by the general public;

(iv) Any substance to the extent it is used in a research laboratory or a hospital or other medical facility under the direct supervision of a technically qualified individual;

(v) Any substance to the extent it is used in routine agricultural operations or is a fertilizer held for sale by a retailer to the ultimate customer.

OSHA Hazard Communication regulations, 29 CFR $\$ 1910.1200(b)$, stipulate exemptions from the requirement to prepare or have available an MSDS.

\section{Reporting Thresholds}

Minimum thresholds have been established for Tier One/Tier Two reporting under Title III, Section 312. These thresholds are as follows:

- For Extremely Hazardous Substances (EHSs) designated under section 302 of Title III, the reporting threshold is 500 pounds or the threshold planning quantity (TPQ), whichever is lower.

- For all other hazardous chemicals for which facilities are required to have or prepare an MSDS, the minimum reporting threshold is 10,000 pounds.

You need to report hazardous chemicals that were present at your facility at any time during the previous calendar year at levels that equal or exceed these thresholds. For instructions on threshold determinations for components of mixtures, see the Chemical Information section on page 9 of these instructions.

\section{When to Submit This Form}

Owners or operators of facilities that have hazardous chemicals on hand in quantities equal to or greater than set threshold levels must submit Tier Two forms by March 1 . 


\section{Hanford Site Tier Two \\ Emergency and Hazardous Chemical Inventory}

\section{Where to Submit This Form}

Send completed Tier Two form to each of the following organizations:

1. Your State Emergency Response Commission.

2. Your Local Emergency Planning Committee.

3. The fire department with jurisdiction over your facility.

\section{Penalties}

Any owner or operator who violates any Tier Two reporting requirements shall be liable to the United States for a civil penalty of up to $\$ 25,000$ for each such violation. Each day a violation continues shall constitute a separate violation.

\section{Completing the Forms}

\section{Reporting Period:}

Enter the appropriate calendar year, beginning January 1 and ending December 31.

\section{Facility Identification:}

Enter the full name of your facility (and company identifier where appropriate). Enter the full street address or state road. If a street address is not available, enter other appropriate identifiers that describe the physical location of your facility (e.g., longitude and latitude). Include city, county, state, and zip code.

Enter the primary Standard Industrial Classification (SIC) code and the Dun \& Bradstreet number for your facility. The financial officer of your facility should be able to provide the Dun \& Bradstreet number. If your firm does not have this information, contact the State or regional office of Dun \& Bradstreet to obtain your facility number or have one assigned.

Owner/Operator:

Enter the owner's or operator's full name, mailing address, and phone number.

\section{Emergency Contact:}

Enter the name, title, and work phone number of at least one local person or office who can act as a referral if emergency responders need assistance in responding to a chemical accident at the facility. Provide an emergency phone number where such emergency information will be available 24 hours a day, every day. The requirement is mandatory. The facility must make some arrangement to ensure a 24 hour contact is available.

\section{Identical Information:}

Check the box indicating identical information, located below the emergency contacts on the Tier Two form, if the current chemical information being reported is identical to that submitted last year. Chemical descriptions, hazards, amounts, and locations must be provided in this year's form, even if the information is identical to that submitted last year. 


\section{Hanford Site Tier Two \\ Emergency and Hazardous Chemical Inventory}

\section{Chemical Information: Description, Hazards, Amounts, and Locations:}

The main section of the Tier Two form requires specific information on amounts and locations of hazardous chemicals, as defined in the OSHA Hazard Communication Standard.

If you choose to indicate that all of the information on a specific hazardous chemical is identical to that submitted last year, check the appropriate optional box provided at the right side of the storage codes and locations on the Tier Two form. Chemical descriptions, hazards, amounts, and locations must be provided even if the information is identical to that submitted last year.

Calculate all amounts as weight in pounds. To convert gas or liquid volume to weight in pounds, multiply by an appropriate density factor. If a chemical is part of a mixture, you have the option of reporting either the weight of the entire mixture or only the portion of the mixture that is a particular hazardous chemical. The option used for each mixture must be consistent with the option used in your Section 311 reporting.

\section{Chemical Description:}

Enter the Chemical Abstract Service registry number (CAS). For mixtures, enter the CAS number of the mixture as a whole if it has been assigned a number distinct from its constituents. For a mixture that has no CAS number, leave this item blank or report the CAS numbers' of as many constituent chemicals as possible. Enter the chemical name or common name of each hazardous chemical. Check box for ALL applicable descriptors: pure or mixture; and solid, liquid, or gas; and whether the chemical is or contains an EHS. If the chemical is a mixture containing an EHS, enter the chemical name of each EHS in the mixture.

If you are withholding the name of a chemical in accordance with criteria specified in Title III, Section 322 , enter the generic class or category that is structurally descriptive of the chemical (e.g., list toluene diisocyanate as organic isocyanate) and check the box marked Trade Secret. Trade secret information should be submitted to EPA and must include a substantiation. Please refer to EPA's final regulation on trade secrecy (53 FR 28772, July 29, 1988) for detailed information on how to submit trade secrecy claims.

\section{Physical and Health Hazards:}

For each chemical you have listed, check all the physical and health hazard boxes that apply. These hazard categories are defined in 40 CFR 370.2. The two health hazard categories and three physical hazard categories are a consolidation of the 23 hazard categories defined in the OSHA Hazard Communication Standard, 29 CFR 1910.1200. 


\section{HAZARD CATEGORY COMPARISON FOR REPORTING UNDER SECTIONS 311 AND 312}

\begin{tabular}{|l|l|}
\hline EPA's hazard categories & \multicolumn{1}{|c|}{ OSHA's hazard categories } \\
\hline Fire Hazard & $\begin{array}{l}\text { Flammable } \\
\text { Combustible Liquid } \\
\text { Pyrophoric } \\
\text { Oxidizer }\end{array}$ \\
\hline $\begin{array}{l}\text { Sudden Release of } \\
\text { Pressure }\end{array}$ & $\begin{array}{l}\text { Explosive } \\
\text { Compressed Gas }\end{array}$ \\
\hline Reactive & $\begin{array}{l}\text { Unstable Reactive } \\
\text { Organic Peroxide } \\
\text { Water Reactive }\end{array}$ \\
\hline $\begin{array}{l}\text { Immediate (Acute) Health } \\
\text { Hazard }\end{array}$ & $\begin{array}{l}\text { Highly Toxic } \\
\text { Toxic } \\
\text { Irritant } \\
\text { Sensitizer } \\
\text { Corrosive } \\
\text { Other hazardous chemicals with an adverse effect with } \\
\text { short term exposure }\end{array}$ \\
\hline $\begin{array}{l}\text { Delayed (Chronic) Health } \\
\text { Hazard }\end{array}$ & $\begin{array}{l}\text { Carcinogen } \\
\text { Other hazardous chemicals with an adverse effect with } \\
\text { long term exposure }\end{array}$ \\
\hline
\end{tabular}

\section{Maximum Amount:}

For each hazardous chemical, estimate the greatest amount present at your facility on any single day during the reporting period. Find the appropriate range value code on Table I. Enter this code as the Maximum Daily Amount.

\section{Average Daily Amount:}

For each hazardous chemical, estimate the average weight in pounds that was present at your facility during the year. To do this, total all daily weights and divide by the number of days the chemical was present on the site. Find the appropriate range value code in Table I. Enter this code as the Average Daily Amount. 
TABLE I - REPORTING RANGES

\begin{tabular}{|c|c|c|}
\hline \multirow{2}{*}{$\begin{array}{l}\text { Range Value } \\
\text { Code }\end{array}$} & \multicolumn{2}{|c|}{ Weight range in pounds } \\
\hline & From & To \\
\hline 01 & 0 &. .99 \\
\hline 02 & 100 & ……………… \\
\hline 03 & 1,000 & 9,999 \\
\hline 04 & 10,000 & 99,999 \\
\hline 05 & 100,000 & 999,999 \\
\hline 06 & $1,000,000$ & 9,999,999 \\
\hline 07 & $10,000,000$ & 49,000,000 \\
\hline 08 & $50,000,000$ & ….............. 99,999,999 \\
\hline 08 & $100,000,000$ & …............. 499,999,999 \\
\hline 10 & $500,000,000$ & … \\
\hline 11 & 1 billion & ......... higher than 1 billion \\
\hline
\end{tabular}

Number of Days On-Site:

Enter the number of days that the hazardous chemical was found on-site.

\section{Storage Codes and Storage Locations:}

List all non-confidential chemical locations in this column, along with storage types/conditions associated with each location. Please note that a particular chemical may be located in several places around the facility. Each row of boxes followed by a line represents a unique location for the same chemical.

For each location, find the appropriate codes for defining the storage types (from Table II) and pressure and temperature conditions (see Table III). Enter the applicable code for the storage type in the first box, the pressure code in the second box, and the temperature code in the third box. 
TABLE II -- STORAGE TYPES

\begin{tabular}{|c|l|c|l|}
\hline Codes & Types of Storage & Codes & Types of storage \\
\hline A & Above ground tank & J & Bag \\
\hline B & Below ground tank & K & Box \\
\hline C & Tank inside building & L & Cylinder \\
\hline D & Steel drum & M & Glass bottles or jugs \\
\hline E & Plastic or non-metallic drum & N & Plastic bottles or jugs \\
\hline F & Can & O & Tote bin \\
\hline G & Carboy & P & Tank wagon \\
\hline H & Silo & Q & Rail car \\
\hline I & Fiber drum & R & Other \\
\hline
\end{tabular}

TABLE III--TEMPERATURE AND PRESSURE CONDITIONS

\begin{tabular}{|c|l|c|l|}
\hline Codes & Pressure conditions & Codes & Temperature conditions \\
\hline 1 & Ambient pressure & 4 & Ambient temperature \\
\hline 2 & Greater than ambient pressure & 5 & Greater than ambient temperature \\
\hline 3 & Less than ambient pressure & 6 & $\begin{array}{l}\text { Less than ambient temperature, } \\
\text { but not cryogenic }\end{array}$ \\
\hline & & 7 & Cryogenic conditions \\
\hline
\end{tabular}

\section{Storage Locations:}

Provide a brief description of the precise location of the chemical, so that emergency responders can locate the area easily. You may find it advantageous to provide the optional site plan or site coordinates as explained below. For each chemical, indicate at a minimum the building or lot. Additionally, where practical, the room or area may be indicated. You may respond in narrative form with appropriate site coordinates or abbreviations. If the chemical is present in more than one building, lot, or area location, continue your responses down the page as needed. If the chemical exists everywhere at the plant site simultaneously, you may report that the chemical is ubiquitous at the site. 


\section{Banford Site Tier Two \\ Emergency and Hazardous Chemical Inventory}

\section{Optional attachments:}

If you choose to attach one of the following, check the appropriate Attachments box at the bottom of the Tier Two form.

a. A site plan with site coordinates indicated for buildings, lots, areas, etc. throughout your facility.

b. A list of site coordinate abbreviations that correspond to buildings, lots, areas, etc. throughout your facility.

c. A description of dikes and other safeguard measures for storage locations throughout your facility.

\section{Confidential Information:}

Under Title III, Section 324, you may elect to withhold location information on a specific chemical from disclosure to the public. If you choose to do so, enter the word "confidential" in the Non-Confidential Location section of the Tier Two form on the first line of the storage locations. On a separate Tier Two Confidential Location Information Sheet, enter the name and CAS number of each chemical for which you are keeping the location confidential. Enter the appropriate location and storage information, as described above for non-confidential locations. Attach the Tier Two Confidential Location Information Sheet to the Tier Two form. This separates confidential locations from other information that will be disclosed to the public.

Certification:

Instructions for this section are included on page one of these instructions. 


\section{BUILDING INDEX}

$100 \mathrm{~B} / \mathrm{C}$ Area (map on page 61)

$105 \mathrm{C}-12,37$

$182 \mathrm{~B}-21,53$

Maintenance trailer $-23,28$

100D/DR Area (map on page 62)

100DR-1 Operable Unit - $11,21,30,37$, $41,45,51$

105DR -12

$190 \mathrm{DR}-1,14,45,48$

$100 \mathrm{H}$ Area (map on page 63)

100-HR-3 Pump \& Treat - 57

100K Area (map on page 64)

100-KR-4 Operable Unit - 57

$105 \mathrm{KE}-4,10,48$

$105 \mathrm{KW}-4,10,48$

$142 \mathrm{~K}-41$

$165 \mathrm{KE}-4,51$

$1706 \mathrm{KE}-4,31,33,53,57$

$1717 \mathrm{~K}-4,12,23,41$

$1724 \mathrm{~K}-30,51$

$1724 \mathrm{KA}-11$

$1724 \mathrm{~KB}-4,16,33,37,41$

$183 \mathrm{KE}-15,30$

$190 \mathrm{KE}-5,23,24,30,33,45,51,57$

$190 \mathrm{KW}-5$

$100 \mathrm{~N}$ Area (map on page 65 )

$105 \mathrm{~N}-19$

$105 \mathrm{NB}-15,19,30,51,59$

$1143 \mathrm{~N}-26$

$1143 \mathrm{NA}-51$

$1330 \mathrm{~N}-1,19,59$

$1512 \mathrm{~N}-4,16,33,37$

$1515 \mathrm{~N}-4,37$

$1714 \mathrm{~N}-51,53,57$

$1714 \mathrm{NB}-41$

$1723 \mathrm{~N}-1,16,41$

$183 \mathrm{~N}-3,15,51,53$

Equipment Pool -21

MO425 - 19, 36, 40

$\mathrm{N}$ Springs -45
200E Area (map on page 66)

$2025 \mathrm{E}-5,10,12,26,29,31,33,41,45$, $46,51,53,54,57$

$204 \mathrm{AR}-21,54$

$209 \mathrm{E}-5,12,42$

$2101 \mathrm{HV}-1,5,17,26,33,42$

$2101 \mathrm{M}-1,5,10,12,15,16,17,20,24$, $26,30,33,37,42,48,50,54,59$

$212 \mathrm{H}-21,37,42$

$225 \mathrm{E}-42$

$241 \mathrm{~A}-17$

$241 \mathrm{~A} 401-24,46,55$

$241 \mathrm{AN}-6,17$

$241 \mathrm{AP}-6,17,34$

$241 \mathrm{AW}-6,16$

$241 A Y-6$

$241 \mathrm{AZ}-46$

$241 B X-6$

$241 \mathrm{BY}-17$

$241 \mathrm{C}-6$

$242 \mathrm{~A}-6,13,21,34,43,52$

$242 \mathrm{~A} 81-13$

$242 \mathrm{AC}-15,34,38,43$

$243 \mathrm{G} 4-10$

$243 \mathrm{G} 6-10$

$2703 \mathrm{E}-7,17,24,34,46,52$

$2711 \mathrm{E}-7,20,23,26,30,34,38,43$

$2715 \mathrm{AW}-24,26,49,52$

$2716 \mathrm{E}-7,17,38$

$2721 \mathrm{E}-20,21$

$2721 \mathrm{EA}-7,11,18,31,38,43,46$

$2727 \mathrm{E}-34$

$272 \mathrm{AW}-7,26,35,38,43,46$

272BA - 52

$275 \mathrm{E}-18,43$

$277 \mathrm{~A}-7,18,25,35,38,46$

$283 \mathrm{E}-3,15$

$284 \mathrm{E}-16,52$

$\mathrm{MO} 269-45$

$6290-9,19,40,45$

HTS Pipeyard - 3, 9, 10, 12, 22, 27, 36, $40,45,49$ 
200E Area (continued)

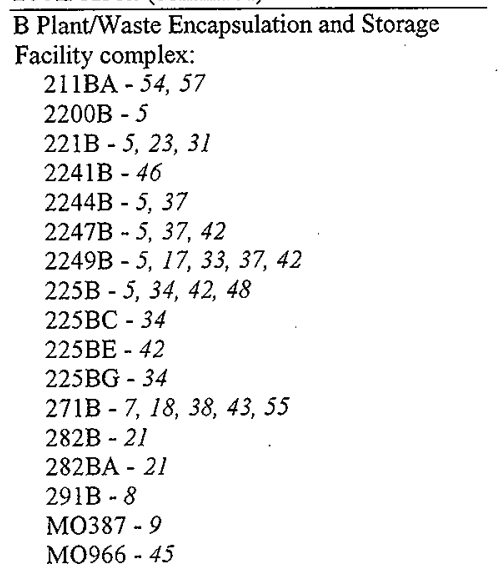

\section{Central Fire Station Complex} and surrounding locations On Route 3 between 200E and 200W Areas (map on page 60)
$607-49,59$
$609 \mathrm{~A}-19,40,44,45$
$609 \mathrm{C}-11,19$
$609 \mathrm{D}-19,40$
$609 \mathrm{G}-19$
$616-14,48,49,50$

\section{W Area (map on page 67)}

200-UP-1 Treatability Test Site - 12

200-ZP-1 Pump and Treat - 12, 16,21,

$33,41,52$

200-ZP-2 ERA - 12, 16, 33, 41, 51

$201 \mathrm{~W}-30$

$202 \mathrm{~S}-33,41$

$222 \mathrm{~S}-1,5,10,13,17,20,29,30,31,33$, $37,42,48,51,54,57$

222SA - $1,5,10,11,24,26,28,29,31$, $37,48,51,54,57$

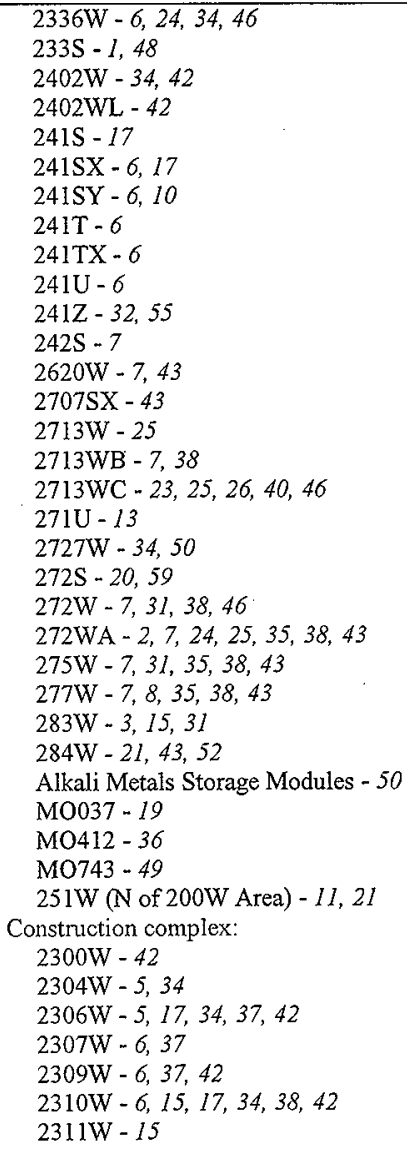


200W Area (continued)

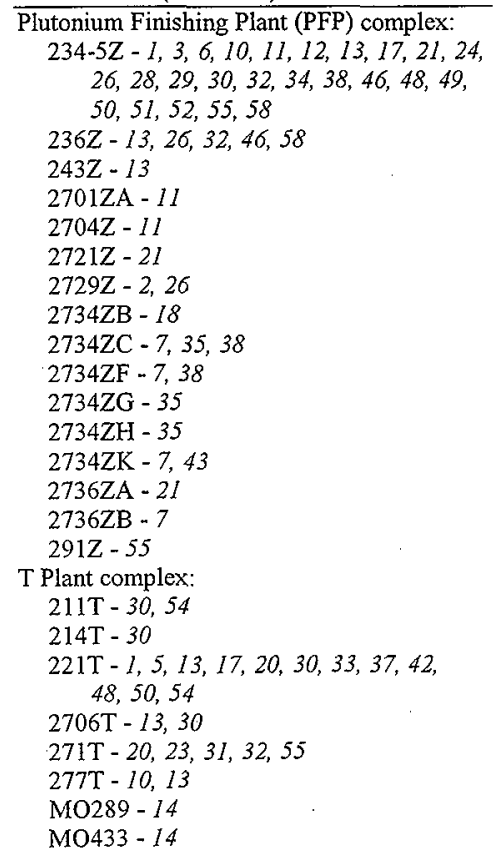

Weather Station Complex, Northeast of 200W Area (map on page 67) $622 \mathrm{R}-2,9,19,27,29,32,36,45$, $53,56,58$

\begin{tabular}{l} 
Waste Sampling and Characterization \\
Facility (WSCF), East of 200W Area \\
(map on page 67) \\
\hline $6265-9,19,36,40,45$ \\
$6266-2,14,20,27,28,29,32,47,49$, \\
$53,56,58$ \\
$6268-32,56$ \\
$6269-14,32,56,58$
\end{tabular}

Environmental Restoration Disposal Facility, Southeast of $200 \mathrm{~W}$ Area (map on page 60)

$6618-22,31$

300 Area (map on page 68)

$305-2,8,18,26,27,29,35,38,43$, 47,49

$305 \mathrm{~A}-8,35,39,47$

$306 \mathrm{E}-2,3,8,12,13,18,24,25,29,32$, $35,39,44,47,49,52,55,58$

$315-3,15,58$

$324-2,8,27,38,44$

$325-8,35,39$

$327-2,8,31,32,47,56$

$328-8,18,35,39,44,59$

$331-39,56$

$331 \mathrm{C}-11,15,35,39,47$

$331 \mathrm{D}-15,20,52,56$

$331 \mathrm{H}-44$

$333-8,44$

$335-50$

$337-35$

$337 \mathrm{~B}-50$

$350-8,35,39$

$3621-66-22$

$3621 \mathrm{D}-22,23,27$

$3705-47,52,56,58$

$3707 \mathrm{E}-27,49$

$3709 \mathrm{~A}-18,39$

$3712-8$

$3717 \mathrm{~B}-8,13,18,24,36,39,52$

$3718 \mathrm{G}-2,15,27,31,52$

$3718 \mathrm{M}-50$

$3718 \mathrm{~N}-44$

$3722-8,38,44$

$3746 \mathrm{D}-3,47,52,58$

$382-52,56$

$382 \mathrm{~B}-22$

$384-8,22,28,36,39,44,53$

Locations near 300 Area

(map on page 60)

300-FF-1 Operable Unit, north of area $-8,21,35,38,43,46$

3020 , south of area $-22,25,47,55$

310 , north of area $-27,28,29,56$

Pit 6 , west of area $-28,58$ 
400 Area (map on page 69)

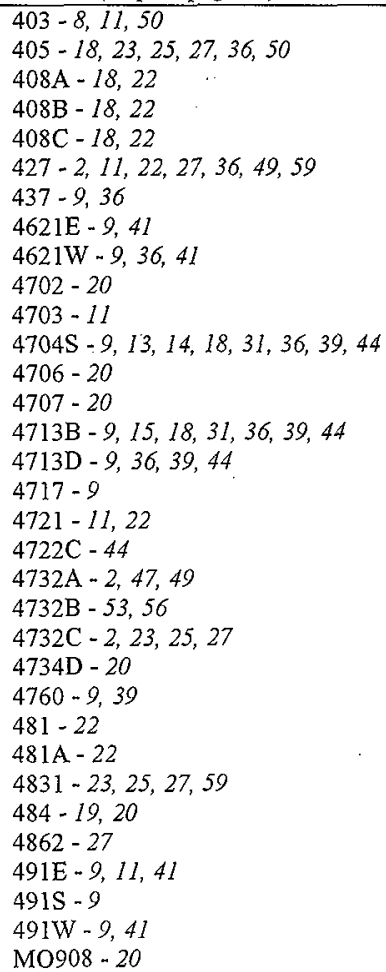

\section{cy and Hazardous Chemical Inventory}

1100 Area (map on page 70 )

$1161-33,41$

$1162-19$

$1168-4,15,16,19,33,37$

$1171-4,15,16,19,21,23,26,30,33$, $37,41,53$

$1171 \mathrm{C}-11,23,26,30,45$

I $176-23,26$

100 Area Fire Station Complex

On corner of Routes 1 and Route 4N

(map on page 60)

$609-19,44$

$613-40$

Hazardous Materials Management and Emergency Response (HAMMER)

Facility (map on page 60)

$6090-44$

$6092-39,44$

$6092 \mathrm{I}-53,56,58$

$6092 \mathrm{~N}-44$

Patrol Training Academy

(map on page 60)

Ammunition conex - 4

MO001 - 40

$\mathrm{MO} 302$ - 40

\section{Ubiquitous locations}

Site building systems -15

Site electrical transformers - 24, 31, 59 


\section{CHEMICAL INDEX}

\section{Chemical Name [CAS number]}

Page(s)

Aluminum oxide [1344-28-1] .. 1-3

Aluminum sulfate dihydrate [10043-0 I-3]

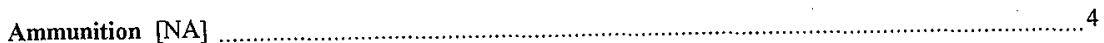

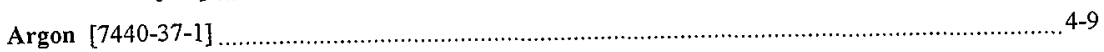

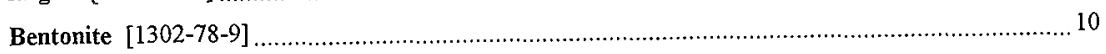

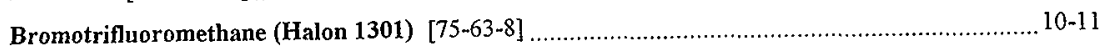

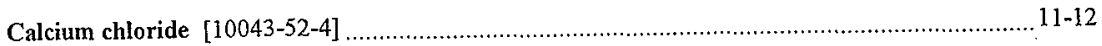

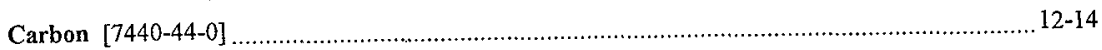

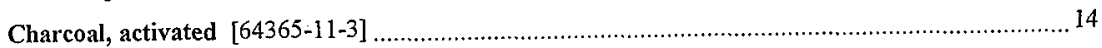

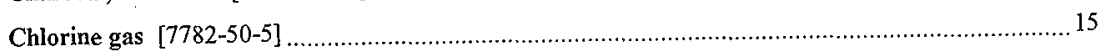

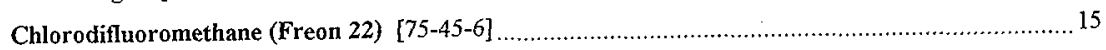

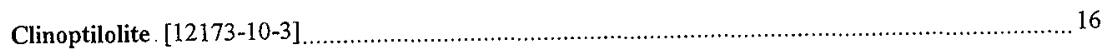

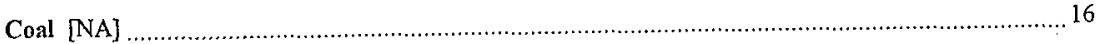

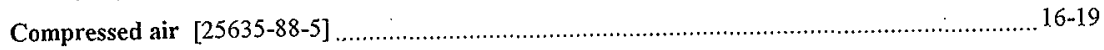

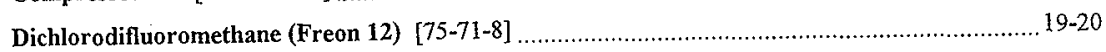

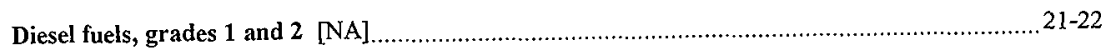

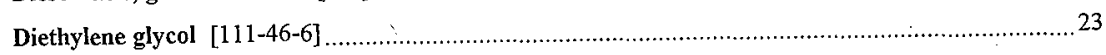

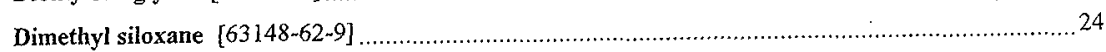

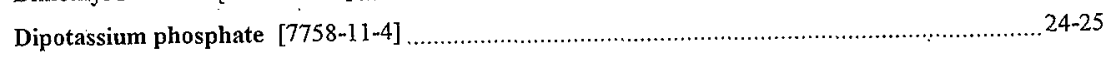

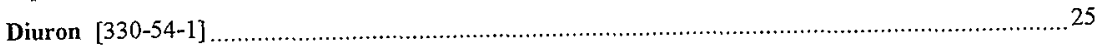

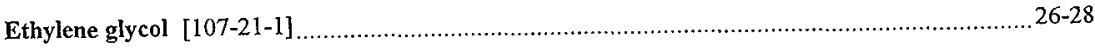

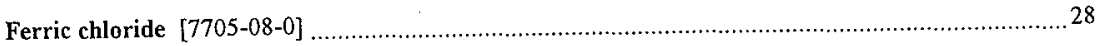

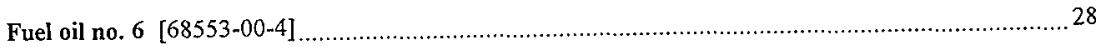

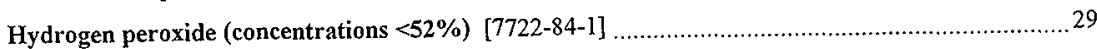

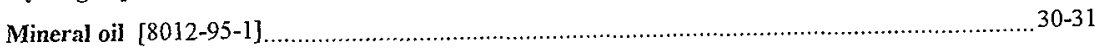

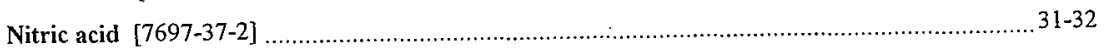

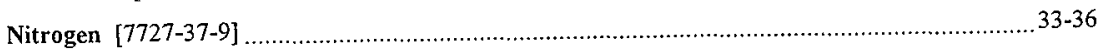


Oxygen [7782-44-7] $37-40$

Paraquat dichloride [1910-42-5]... 40

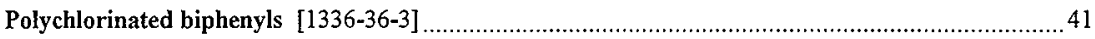

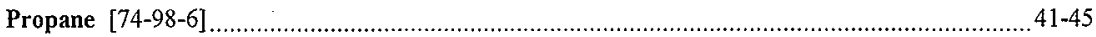

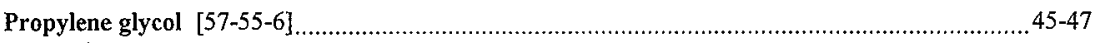

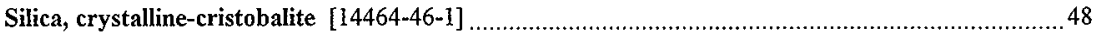

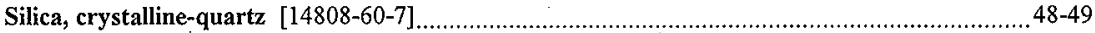

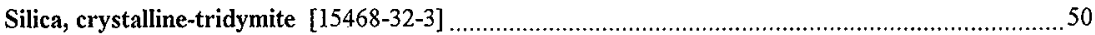

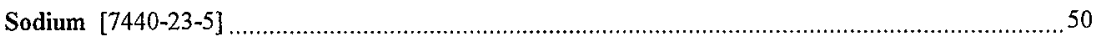

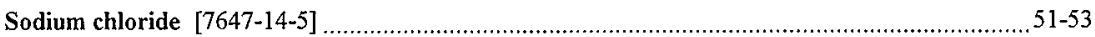

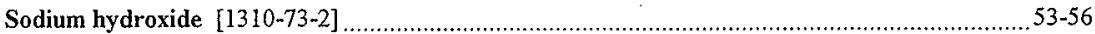

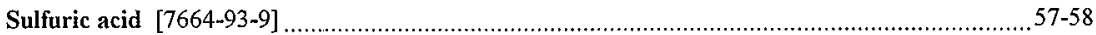

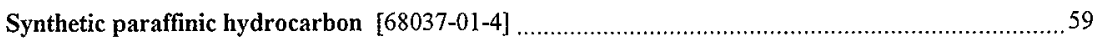

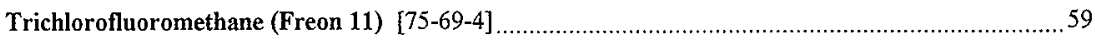




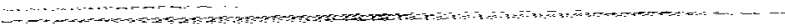

-

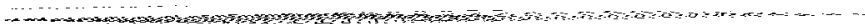

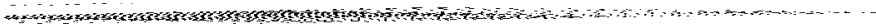

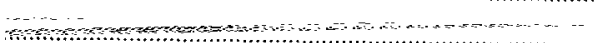

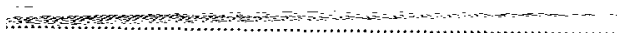

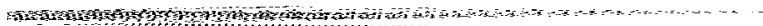

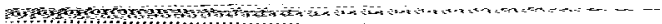

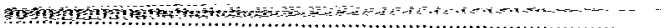

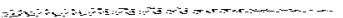

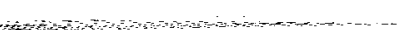

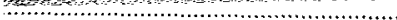

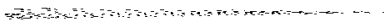

and

(1)

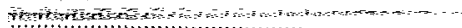

Fon

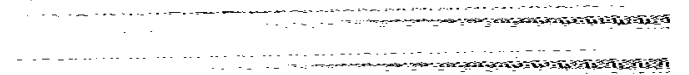

-

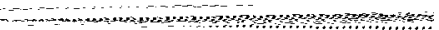

- . n

-100 -

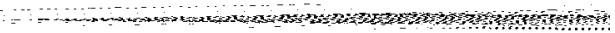

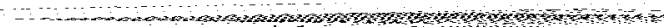




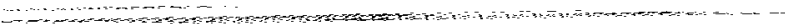

-

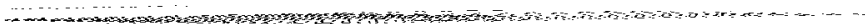

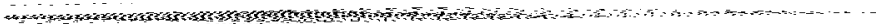

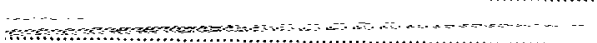

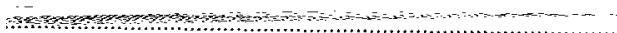

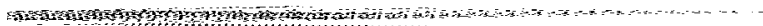

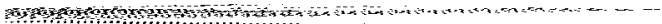

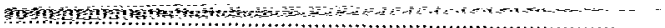

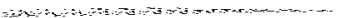

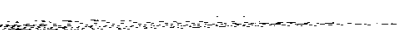

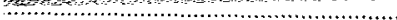

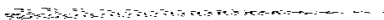

and

(1)

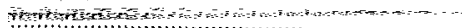

Fon

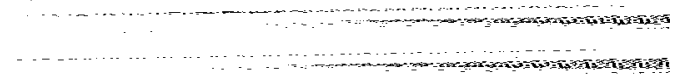

-

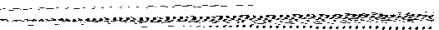

- . n

-100 -

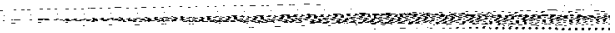

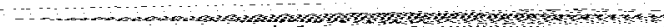


DISTRIBUTION, continued

Number

Of Copies

3

Central Files

EDMC

4

1

2

7

5

ONSITE RECIPIENTS

R. J. Landon

Patrol Operations Center

Fluor Daniel Hanford. Inc.

W. D. Adair

W. E. Toebe
B1-07

A3-93

H6-08

H0-15

HO-O9

H9-0I

$\mathrm{H} 0-02$

S2-98

$\begin{array}{ll}\text { E. D. Woodard, Medical Director } & \mathrm{H} 1-53 \\ \text { S. M. McInturff, HEHF EP Coordinator } & \mathrm{H} 1-77\end{array}$

$\begin{array}{ll}\text { E. D. Woodard, Medical Director } & \mathrm{H} 1-53 \\ \text { S. M. McInturff, HEHF EP Coordinator } & \mathrm{H} 1-77\end{array}$

H6-21

M. E. Brown, PHMC EP Coordinator

A3-05

H6-23

B3-15

$\begin{array}{ll}400 \text { Area Incident Command Post } & \text { N2-34 } \\ \text { G. A. Aldridge (2), POC for } & \text { A } 0-20\end{array}$

Hanford Emergency Operations Center

Alternate Emergency Operations Center

Pacific Northwest National Laboratory

A. K. Ikenberry

P7-79

E. A. Raney

P7-79

J. B. Schuette, PNNL EP Coordinator

DOE Public Reading Room

K6 44

$\mathrm{H} 2-53$

Hanford Technical Library 
DOE/RL-99-16

DISTRIBUTION, continued

17

U.S. Department of Energy. Richland Operations Office

H. E. Bilson

R3-79

R. F. Christensen

K8-50

C. P. Christenson

A5-55

W. F. Edwards

A2-45

O. A. Farabee

R3-79

L. E. Gilk

A6-35

R. A. Holten

$\mathrm{H} 0-12$

R. N. Krekel (2)

A5-15

J. E. Ollero

A5-18

J. C. Peschong

S7-51

J. E. Rasmussen

A5-15

L. D. Romine

R3-79

E. D. Sellers

S7-41

J. L. Tokarz-Hames, RL EP Coordinator

A5-55

S. H. Wisness

A5-18

J. H. Zeisloft

$\mathrm{H} 0-12$

6

Waste Management Federal Services of Hanford. Inc.

T. A. Burdin

H6-20

J. O. Skolrud

H6-20

A. G. Weiner

H6-21

J. A. Winterhalder

H6-21

D. E. Zaloudek

WMH Legal

H6-20

H6-20 